\title{
Hydrogen Atom Transfer Experiments Provide Chemical Evidence for the Conformational Differences between $C$ - and $O$-Disaccharides
}

\author{
Elisa I. León, ${ }^{[a]}$ Angeles Martín, ${ }^{*[a]}$ Inés Peréz-Martín, ${ }^{[a]}$ Luis M. Quintanal, ${ }^{[a]}$ and \\ Ernesto Suárez ${ }^{*[a]}$
}

Keywords: Carbohydrates / Disaccharides / Conformation analysis / Radical reactions / Hydrogen transfer

The glycopyran-6-O-yl radical promoted hydrogen atom transfer reaction (HAT) between the two pyranose units of $\alpha$ D-Man $p-(1<4)-\alpha-D-G l c p$ and $\alpha$-D-Man $p-(1<4 a)-4 a-\operatorname{carba}-\alpha-$ D-Glc $p$ disaccharides provides supporting chemical evidence for the conformational differences between $O$ - and $C$-glycosyl compounds. In the O-disaccharide the 6-alkoxyl radical, generated under oxidative or reductive conditions, abstracts exclusively the hydrogen at C-5' via a completely regioselective 1,8-HAT reaction. This may be attributable to the conformational restriction of the glycosidic and aglyconic bonds due principally to steric and stereoelectronic effects. On the contrary, very little regioselectivity is observed in the homologous $C$-disaccharide and a mixture of compounds generated by $1,5-, 1,6-$, and 1,8-HAT processes where the abstraction occurs at hydrogen atoms positioned at C-4a, C-1', and C-5', respectively, has been obtained. This study has been extended to simpler $\mathrm{O}$ - and $\mathrm{C}$-glycosides, where the aglycon was a straight $n$-alkyl alcohol tether of five atoms; in general, all of the results obtained are shown to be consistent with a major conformational flexibility of the $C$-glycosidic bond.

\section{Introduction}

$C$-Glycosides have recently attracted considerable synthetic attention in glycoconjugate chemistry because of their promising biological and therapeutical properties and in view of the increased detection of the $C$-glycosidic bond embodied in a variety of natural products. ${ }^{[1]}$ Due to the biological significance of these substances, especially as enzyme inhibitors, the comparison of the conformational behaviour of $C$-glycosides with the naturally occurring $O$ counterparts is an interesting research topic. ${ }^{[2]}$ The conformational studies have so far been carried out by using physical methods, particularly NMR spectroscopy (NOE analysis and coupling constants data) in combination with molecular mechanics and ab initio calculations. The obtained results show that the population distribution of conformers with respect to the orientation around the $C$-glycosidic linkages is mainly controlled by steric interactions. In contrast for natural $O$-glycosides, additional stereoelectronic and steric factors contribute to an increased rigidity around the glycosidic linkage.

In a previous paper from this laboratory, we described the 1,8-hydrogen atom transfer $(1,8$-HAT) between the two glucopyranose units of an $\alpha-\square-G l c p-(1<4)-\beta-\square-G l c p$ disaccharide ( $\square$-maltose) through a nine-membered transition

[a] Instituto de Productos Naturales y Agrobioogía del C. S. I.C., Carretera de la Esperanza 3, 38206 La Laguna, Tenerife, Spain Fax: +34-922-260-135

E-mail: angelesmartin@ipna.csic.es esuarez@ipna.csic.es

Supporting information for this article is available on the WWW under http://dx.doi.org/10.1002/ejoc.201000470. state. ${ }^{[3]}$ The process occurs with absolute regioselectivity via an intramolecular radical mechanism in a completely analogous manner to the case described in Scheme $1(\mathbf{A}<\mathbf{B})$. This methodology was later extended to many other $(1<4)$ disaccharide models and may be synthetically useful for the remote functionalization of the $\mathrm{C}-5^{\prime}$ carbon atom or for the specific inversion of the configuration at this centre. ${ }^{[4]}$

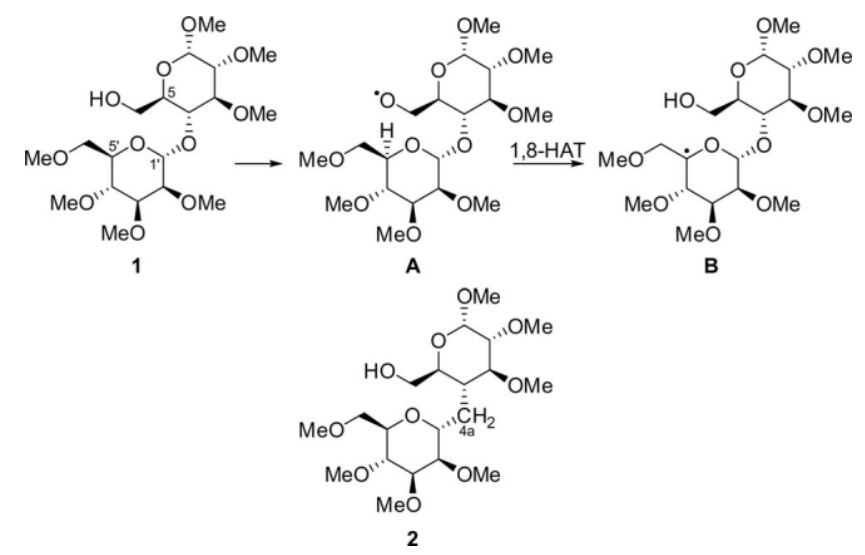

Scheme 1. 1,8-HAT in $\alpha$ - $\square$-Man $p$ - $(1<4)-\beta-\square-G l c p$ (1) $O$-disaccharide system.

\section{Results and Discussion}

During the course of this research, we became aware that this 1,8-HAT reaction, where geometric and stereoelectronic factors dominate, offers an unrivalled opportunity to 
gain further insight into the conformational differences between $O$ - and $C$-glycosides.

To probe the entropic barrier resulting from flexibility around the glycosidic bond in going from the conformationally restricted $O$-disaccharides to the more flexible $C$ disaccharides it was necessary to synthesize a relatively simple model system. In designing $\alpha-\square-\operatorname{Man} p-(1<4)-\alpha-\square-G l c p$ disaccharide 1 and $\alpha-\square-\operatorname{Man} p-(1<4 \mathrm{a})-4 \mathrm{a}-\mathrm{carba}-\alpha-\square-$ Glc $p$ 2as models for the HAT reaction, we were guided by a number of considerations. Firstly, in an $\alpha-\square$-manno configu- ration for the ${ }^{4} C_{1}$ ring II of the disaccharide the 6-O-yl radical is ideally suited to permit hydrogen atom abstrac- tion from almost all positions of the pyranose ring (specifi- cally from C-1', C-2', C-3', and C-5'; C-4a would also be considered in $C$-disaccharides). Secondly, none of the three staggered conformations around the $\Phi_{\mathrm{H}}$ angle for the ${ }^{4} C_{1}$ chair (exo-syn, exo-anti, and non-exo) should undergo substantial steric interaction between the axial substituent at C-2' and the aglycon (scheme of Table 1). The influence of a C-2' equatorial substituent on the conformational equilibrium seems to be especially important in the absence of additional stereoelectronic effects, as in the case of $C$-glycosides. Finally, as determined by NMR and molecular dynamics, the manno- $C$-glycosyl compounds deviate significantly from the behaviour usually observed in $O$-glycosides, where the exo-syn is the preferred conformation, by presenting high percentages of the non-exo rotamer. ${ }^{[2 \mathrm{a}]}$
A preliminary molecular mechanics study of our mannomodels $\mathbf{1}$ and $\mathbf{2}$ is outlined in Table $1{ }^{\left[{ }^{[5]}\right.}$ These results are in general agreement with those previously reported for the totally unprotected disaccharides. ${ }^{[2 a]}$ Comparing the increments of minimized energy observed for the different conformers exo-syn, non-exo and exo-anti for both models a major flexibility of the glycosidic bonds in the $C$-disaccharide is clearly distinguishable. Furthermore, in the $C$ disaccharide 2 a similar energy is observed for the exo-syn and non-exo conformations $\left(\Delta \Delta E\right.$ Š $\left.1 \mathrm{kcalmol}^{-1}\right)$, indicative of the fact that, as commented previously, the non-exo conformation could be significantly populated.

We have calculated the distance between the oxygen at C-6 and the principal extractable protons as indicative of HAT reaction feasibility. It is generally accepted that distances of approx. 2.5-3 $\AA$ are required for the reaction to take place. ${ }^{[6]}$ On the basis of conformational energies and distances the predictable HAT reactions are outlined in the scheme of Table 1 . In the case of $O$-disaccharide $\mathbf{1}$ only a 1,8-HAT reaction by abstraction of the $\mathrm{H}-5^{\prime}$ is expected to be favourable. The situation is more complicated with the $C$-disaccharide 2 and 1,5-, 1,6- and 1,8-HAT processes may be operative via the two exo-syn conformational minima by abstraction of hydrogens $\mathrm{H}_{\mathrm{S}}-4 \mathrm{a}, \mathrm{H}-1^{\prime}$ and $\mathrm{H}-5^{\prime}$, respectively. Moreover, abstraction of hydrogens at $\mathrm{H}^{-1} \mathrm{1}^{\prime}$ and $\mathrm{H}_{\mathrm{S}^{-}}$ $4 \mathrm{a}$ via the non-exo conformation may be also possible. Abstraction at 2' via the energetically disfavoured exo-anti

Table 1. Newman projections for the staggered conformations around the glycosidic torsion angle $\Phi$ in $O$ - and $C$-disaccharides $\mathbf{1}$ and 2. [a]
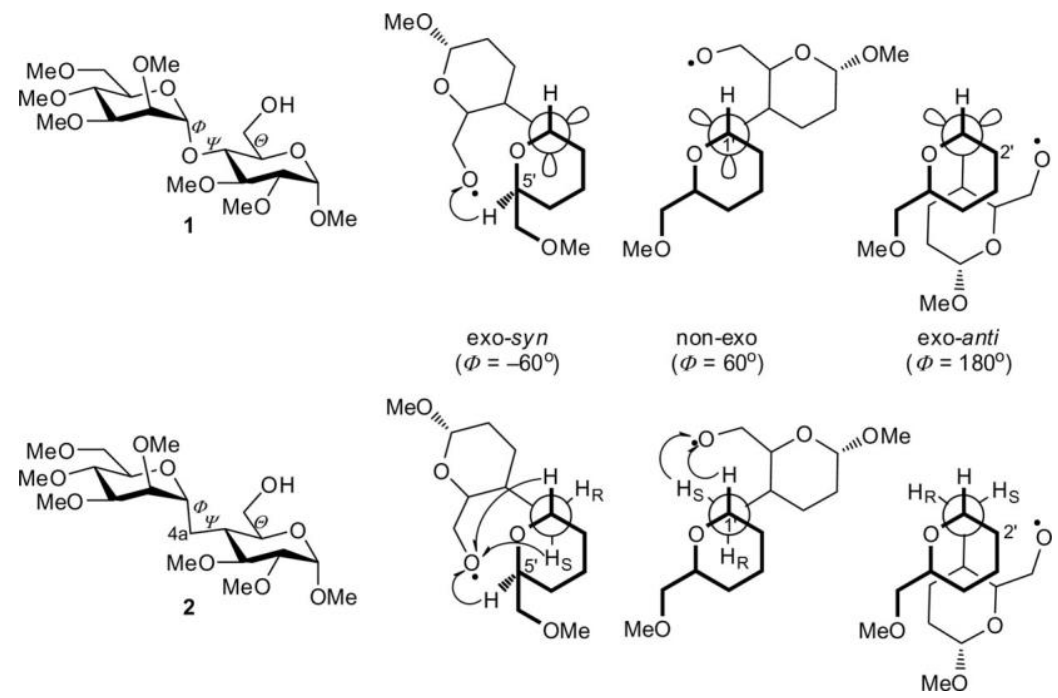

\begin{tabular}{|c|c|c|c|c|c|c|c|c|c|}
\hline Substrate & Conformation $^{[b]}$ & $\Phi\left[\left[^{\circ}\right]^{[c]}\right.$ & $\Psi\left[^{\mathrm{o}}\right]^{[\mathrm{d}]}$ & $\Theta\left[{ }^{\mathrm{o}}\right]^{[\mathrm{e}]}$ & $d_{\mathrm{O}-5^{\prime}}\left[\AA{ }^{[\mathrm{ff}]}\right.$ & $d_{\mathrm{O}-1^{\prime}}[\AA]^{[\mathrm{f}]}$ & $d_{\mathrm{O}-4 \mathrm{a}}[\AA]^{[\mathrm{f}]}$ & $d_{\mathrm{O}-2^{\prime}}\left[\AA \AA^{[\mathrm{ff}]}\right.$ & $\Delta E\left[\mathrm{kcalmol}^{-1}\right]$ \\
\hline \multirow{2}{*}{$O$-Disaccharide 1} & exo-syn & -15.0 & -24.8 & 172.2 & 2.6 & 4.3 & - & 5.4 & 0 \\
\hline & exo-anti & -121.7 & -25.2 & 165.9 & 4.1 & 4.5 & - & 3.2 & 12.9 \\
\hline \multirow[t]{4}{*}{$C$-Disaccharide 2} & exo-syn & -54.7 & 165.4 & 158.6 & 5.8 & 2.6 & 2.7 & 3.7 & 0 \\
\hline & exo-syn & -55.1 & -29.8 & 146.7 & 2.9 & 5.3 & 2.5 & 5.4 & 0.3 \\
\hline & non-exo & 50.1 & 14.9 & 154.9 & 5.1 & 2.9 & 2.7 & 5.1 & 1 \\
\hline & exo-anti & 179.8 & -0.3 & 154.7 & 5.5 & 4.3 & 2.5 & 2.6 & 7.1 \\
\hline
\end{tabular}

[a] The arrows show the hydrogen atoms transfer reactions predicted by molecular mechanics calculations performed with AMBER* force field as implemented in MacroModel. ${ }^{[5]}[\mathrm{b}]$ Staggered conformations around the glycosidic bond $\Phi$. [c] Glycosidic bond, $\Phi=\mathrm{H}-1^{\prime}-$ C-1'-O(C)-C-4. [d] Aglyconic bond, $\Psi=\mathrm{C}-1^{\prime}-\mathrm{O}(\mathrm{C})-\mathrm{C}-4-\mathrm{H}-4$. [e] $\Theta=$ O-6-C-6-C-5-O (Conformation tg). [f] Calculated distance from the $O$-radical to the corresponding hydrogen. In the graphic the methoxyl groups at C-2, C-3, C-2', C-3' and C-4' are omitted for clarity. 
conformation does not seem to be a competitive 1,7-HAT reaction in either $O$-disaccharide $\mathbf{1}$ or $C$-disaccharide 2 . Therefore, one would expect that the HAT reaction on $O$ disaccharide 1 would be very regioselective, while a relatively complex mixture of compounds resulting from different 1,5-, 1,6-, and 1,8-HAT processes would be obtained if the reaction is performed with the $C$-disaccharide 2 .

The required $\alpha-\square-$ Man $p-(1<4)-\alpha-\square-$ Glc $p$ disaccharide 1 was efficiently synthesized by the classical Lewis acid-mediated glycosylation of a suitably protected $\square$-glucopyranose derivative as glycosyl acceptor with 2,3,4,6-tetra- $O$-methyl$\alpha-\square$-mannopyranosyl trichloroacetimidate ${ }^{[7]}$ as glycosyl donor, as described in the Supporting Information. ${ }^{[8]}$

The analogous $C$-disaccharide $\alpha-\square-\operatorname{Man} p-(1<4 a)-4 a-$ carba- $\alpha-\square-$ Glcp 2 was prepared as described in Scheme 2 employing a $\mathrm{SmI}_{2}$-mediated $C$-glycosylation between the sulfone $\mathbf{3} \boldsymbol{\alpha}$ and the aldehyde $\mathbf{4} \boldsymbol{\alpha} .{ }^{[9]}$ The pyridylsulfone $\mathbf{3}$ was synthesized by $S$-glycosidation of 2,3,4,6-tetra- $O$-methyl- $\alpha$ $\square$-mannopyranosyl trichloroacetimidate with 2-mercaptopyridine and subsequent oxidation according to well-established experimental procedures. ${ }^{[9 \mathrm{~g}]}$ The aldehyde $\mathbf{4} \boldsymbol{\alpha}$ was in turn prepared from the known ketone $\mathbf{5}^{[4 a]}$ via slightly modified literature procedures (see Supporting Information). ${ }^{[10]}$ This transformation requires Wittig methylenation to give olefin 6 and hydroboration using 9-BBN to afford a separable mixture of alcohols $7 \boldsymbol{\alpha}$ and $7 \boldsymbol{\beta}$ in a 1:2 ratio which can be individually oxidized with PCC to aldehydes $\mathbf{4 \alpha}$ and $\mathbf{4 \beta}$ (Scheme 2). The major undesired axial aldehyde $\mathbf{4 \beta}$ with the wrong stereochemistry at C-4 was recycled into the required equatorial $\mathbf{4} \alpha$ by base-catalyzed isomerisation.
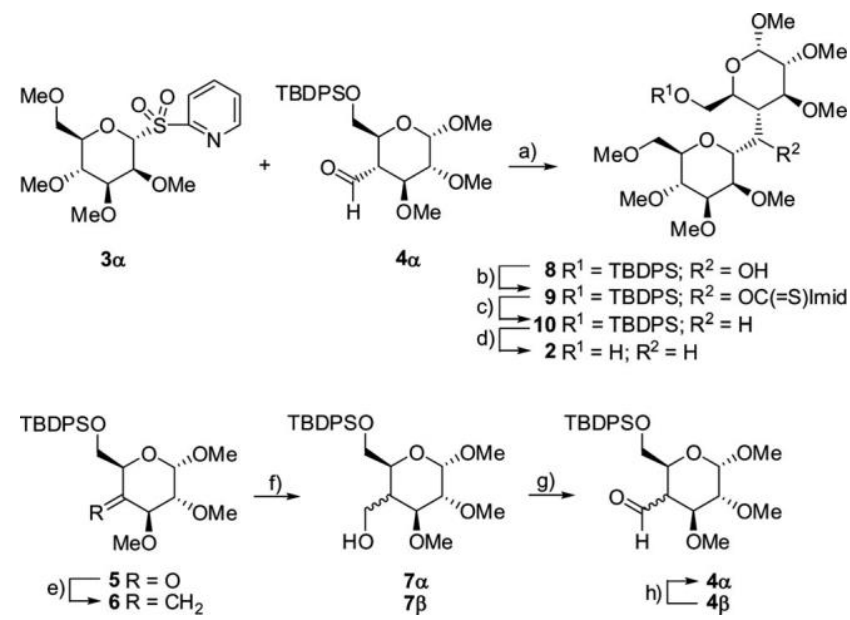

Scheme 2. Synthesis of $C$-disaccharide $\alpha$ - $\square-M a n p-(1<4 a)-4 a-$ carba- $\beta-\square-G l c p$ (2); a) sulfone $\mathbf{3} \boldsymbol{\alpha}$ (2 equiv.), aldehyde $\mathbf{4 \alpha}$ (1 equiv.), $\mathrm{SmI}_{2}$ (5.3 equiv.), THF, r.t., $20 \mathrm{~min}, 42 \%$; b) 1,1-thiocarbonyldiimidazole (20 equiv.), $\mathrm{MeCN}$, reflux, overnight, $79 \%$; c) $\mathrm{Ph}_{3} \mathrm{SnH}$ (3.3 equiv.), pentafluorophenol (2.5 equiv.), AIBN (0.1 equiv.), toluene, reflux, 1.5 h, $92 \%$; d) TBAF (1.2 equiv.), THF, r.t., 7 h, $90 \%$; e) methyltriphenylphosphonium bromide (6 equiv.), $n \mathrm{BuLi}, \mathrm{THF}$, $-78{ }^{\circ} \mathrm{C}, 95 \%$; f) 9-BBN (9 equiv.), THF, reflux, 4 h; then $\mathrm{NaOH}$, $\mathrm{H}_{2} \mathrm{O}_{2}(30 \%), 0{ }^{\circ} \mathrm{C}, 0.5 \mathrm{~h}, 7 \boldsymbol{\alpha}(30 \%)$ and $7 \boldsymbol{\beta}(57 \%)$; g) $\mathrm{PCC}, \mathrm{CH}_{2} \mathrm{Cl}_{2}$, r.t., $2-3 \mathrm{~h}, 74-86 \%$; h) TEA, $60^{\circ} \mathrm{C}, 9 \mathrm{~h}, 75 \%$. AIBN = azobisisobutyronitrile, $\mathrm{TBAF}=$ tetrabutylammonium fluoride.
After the reductive samariation the $C$-disaccharide $\mathbf{8}$ was isolated in $42 \%$ yield as a single stereoisomer, no other isomers being detected by NMR in the crude reaction mixture. ${ }^{[11]}$ The H-1'-H-2' coupling constant could not be measured by inspection of the ${ }^{1} \mathrm{H}$ NMR spectrum by overlapping of the signals, but the $\mathrm{C}-1^{\prime}$ stereochemistry was confirmed by NOE interactions between the bridge hydrogen and H-5' among others. The configuration of the newly formed hydroxy group was of no consequence and thus not determined. The deoxygenation of this hindered secondary alcohol was accomplished by triphenyltin hydride reduction of the $1 H$-imidazole-1-carbothioate intermediate 9 following a previously reported procedure. ${ }^{[9 \mathrm{~h}]}$ Deprotection of the silyl ether 10 with TBAF afforded the required alcohol 2. In this compound, it was possible to measure the coupling constant between H-1'-H-2' $(J=4.5 \mathrm{~Hz})$ and it is in good agreement with those described for $\alpha$ - $C$-glycosides of $\square-$ manno configuration (vide infra). Moreover, to study the HAT reaction under reductive conditions and acquire additional insight into its mechanism, the phthalimido derivatives 13 (Scheme 3 ) and $\mathbf{2 2}$ (Scheme 5) were also prepared from the corresponding alcohol and $N$-hydroxyphthalimide under Mitsunobu conditions in accordance with a previously described protocol.[12]

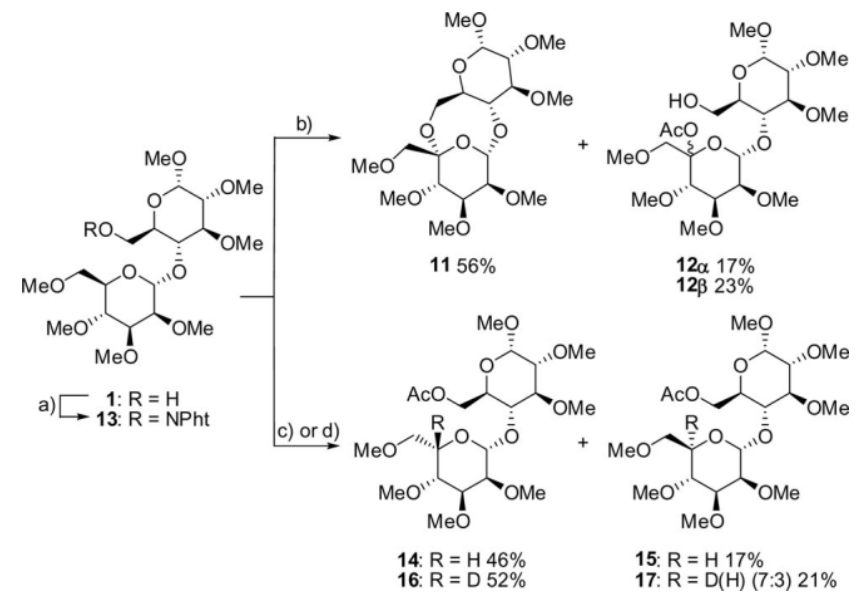

Scheme 3. HAT of $\alpha-\square-\operatorname{Man} p-(1<4)-\beta-\square-G l c p$ (1); a) DEAD $\quad(2.5$ equiv.), $N$-hydroxyphthalimide (2 equiv.), $\mathrm{PPh}_{3}$ (2.5 equiv.), THF, r.t., $1 \mathrm{~h}, 46 \%$; b) DIB (2.5 equiv.), iodine (1 equiv.), $\mathrm{CH}_{2} \mathrm{Cl}_{2}$, reflux, $3 \mathrm{~h}$; c) $n \mathrm{Bu}_{3} \mathrm{SnH}$ (2 equiv.), $\mathrm{AIBN}$ (0.4 equiv.), $\mathrm{PhH}$, reflux, $3.5 \mathrm{~h}$; then $\mathrm{Ac}_{2} \mathrm{O}$, Py, r.t., $4 \mathrm{~h}$; d) $n \mathrm{Bu}_{3} \mathrm{SnD}$ ( 2.5 equiv.), $\mathrm{AIBN}$ (0.4 equiv.), $\mathrm{PhH}$, reflux, $3.5 \mathrm{~h}$; then $\mathrm{Ac}_{2} \mathrm{O}$, Py, r.t., 4 h. DEAD = diethyl azodicarboxylate, $\mathrm{DIB}=($ diacetoxyiodo $)$ benzene, $\mathrm{NPht}=$ phthalimide.

Firstly, we carried out the HAT reaction with the $O$-disaccharide 1 under oxidative conditions by treatment with the DIB/iodine system, as shown in Scheme 3. ${ }^{[3,4]}$ The reaction afforded the trioxocane $\mathbf{1 1}$ as the main product $(56 \%)$ and a partially separable mixture of isomeric acetates $\mathbf{1 2} \boldsymbol{\beta}$ (23\%) and $\mathbf{1 2 \alpha}(17 \%)$. It is evident that all three compounds are formed by the same $\mathrm{C}-5$ ' radical intermediate promoted by the alkoxyl radical through an intramolecular 1,8-HAT reaction (Table 2, entry 1 ). Additional mechanistic features include oxidation of this newly formed $C$-radical to an oxonium ion by an excess of reagent, and finally reac- 
tion with different nucleophilic trapping agents. The intramolecular reaction with the primary alcohol at C-6 affords trioxocane $\mathbf{1 1}$ while acetates $\mathbf{1 2}$ are formed intermolecularly by reaction with acetate anions coming from the DIB.

Table 2. HAT reactions in $\alpha-\square$-mannopyranosyl glycosides.

\begin{tabular}{|c|c|c|c|c|c|c|}
\hline Entry & Substrate & Process & $\begin{array}{c}\text { abst. 5' } \\
{[\%]}\end{array}$ & $\begin{array}{l}\text { abst. } \\
\text { 1' [\%] }\end{array}$ & $\begin{array}{c}\text { abst. } 4 \mathrm{a} \\
{[\%]}\end{array}$ & $\begin{array}{l}\text { red. O } \\
{[\%]^{[a]}}\end{array}$ \\
\hline 1 & 1 & ox. & 96 & n.d. & - & - \\
\hline 2 & 13 & red. ${ }^{[b]}$ & 67 & n.d. & - & 6 \\
\hline 3 & 2 & ox. & 32 & 41 & 9 & - \\
\hline 4 & 22 & red. ${ }^{[\mathrm{b}]}$ & 17 & 31 & 6 & 14 \\
\hline 5 & 28 & ox. & 38 & 14 & - & - \\
\hline 6 & 35 & red..$^{\mathrm{b}]}$ & 22 & 14 & - & 21 \\
\hline 7 & 31 & ox. & n.d. & 55 & 32 & - \\
\hline 8 & 42 & red..$^{[b]}$ & n.d. & 32 & 49 & 12 \\
\hline
\end{tabular}

[a] Reduction of the alkoxyl radical prior to the abstraction reaction. [b] The yields of the reductive processes were calculated from the $n \mathrm{Bu}_{3} \mathrm{SnD} / \mathrm{AIBN}$ experiment to evaluate the products with retention of configuration and the percentage of reduction of the alkoxyl radical prior to abstraction; n.d. = not detected.

The stereochemistry of C-5' and the related conformation of ring II in acetates $\mathbf{1 2}$ deserve a brief comment. The observed pattern of $J$ coupling constants between the ring protons indicates that, as expected, in $\mathbf{1 2} \beta$ the $\beta$ - $\square$-gulo ring adopts a ${ }^{1} C_{4}$ chair conformation while in the case of $\mathbf{1 2 \alpha}$ the $\alpha$ - $\square$-manno ring remains unchanged in a ${ }^{4} C_{1}$ chair.

Next, the HAT reaction was carried out under reductive conditions with the phthalimide $\mathbf{1 3}$ by treatment with $n \mathrm{Bu}_{3} \mathrm{SnH} / \mathrm{AIBN}$. In this case two compounds were obtained after acetylation to facilitate their chromatographic separation: disaccharide 14 (46\%) formed by hydrogen abstraction at $\mathrm{C}-5^{\prime}$ and radical quenching by the stannane with inversion of configuration and the acetyl derivative of the precursor alcohol 15 (17\%) which, evidently, arises either by abstraction and retention of configuration at C-5' or simply by reduction of the 6-O-yl radical prior to the abstraction reaction, or most probably by a combination of these two mechanisms. In the inverted disaccharide 14 the initial $\alpha-\square$ mannopyranosyl moiety has been transformed into a $\beta$ - $\square$ gulopyranosyl derivative and consequently the pyranose ring has changed from ${ }^{4} C_{1}$ to ${ }^{1} C_{4}$ conformation as can be easily observed by ${ }^{1} \mathrm{H}$ NMR spectroscopy.

The reduction of phthalimide $\mathbf{1 3}$ using $n \mathrm{Bu}_{3} \mathrm{SnD}$ as reagent showed, after careful analysis of the isotopic distribution, a total substitution of deuterium at C-5' in compound 16 while $70 \%$ of deuterium labelling was found in the alcohol 17, and therefore the direct reduction of the 6-O-yl radical is responsible for the $30 \%$ of unlabelled molecules. ${ }^{[13]}$ The deuterium is only incorporated at C-5', and there is no observable deuterium scrambling at other positions of the molecule within NMR detection limits. These results have also allowed us to establish that also under these reductive conditions only 1,8 -HAT is taking place and that the abstraction at C-5' occurs in $67 \%$ yield with an inversion/retention ratio of approximately 3.5:1 (Table 2, entry 2).
We can conclude that the HAT reaction of $\alpha-\square-M a n p-$ $(1<4)-\alpha-\square-$ Glc $p$ disaccharide 1 occurred with complete regioselectivity by exclusive abstraction of the hydrogen atom at C-5' in nearly quantitative global yield (96\%) under oxidative conditions. Within the limits of NMR detection, no products coming from abstraction of other hydrogen atoms were detected in the reaction mixture.

The HAT reaction of the $C$-disaccharide $\alpha$ - $\square$-Manp( $1<4 a)-4 a-c a r b a-\beta-\square-G l c p$ (2) proved to be a much more complex process from the mechanistic standpoint. Under oxidative conditions the expected 1,3-dioxocane $\mathbf{1 8}$ coming from the abstraction of the H-5' was isolated, but only in $30 \%$ yield (Scheme 4 ). The major product was now the dioxaspirobicycle 19 generated by 1,6-HAT between the alkoxyl radical and the hydrogen at $\mathrm{C}-1{ }^{1} \cdot{ }^{[14]}$ Moreover, two minor iodinated compounds $\mathbf{2 0}$ and $\mathbf{2 1}$ which were plausibly formed by two consecutive hydrogen abstractions were also isolated. The alkoxyl radical abstracted initially a hydrogen atom from the interconnecting C-4a through a very favourable 1,5 -TS to give a $\delta$-iodohydrin intermediate. This intermediate would then give by a second abstraction, either 20 through a 1,6-HAT or 21 by a 1,7-HAT, competitively.

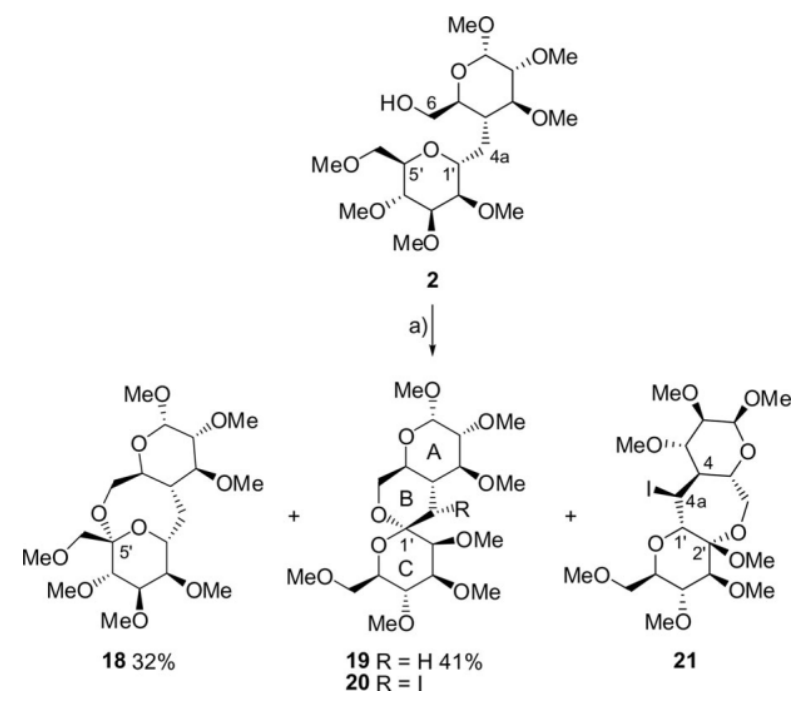

Scheme 4. Oxidative HAT of $\alpha-\square-M a n p-(1<4 a)-4 a-c a r b a-\beta-\square-G l c p$ (2); a) DIB (1.7 equiv.), iodine (1 equiv.), $\mathrm{CH}_{2} \mathrm{Cl}_{2}, h v$, r.t., $1.5 \mathrm{~h}$.

Some stereochemistry and conformational aspects of these compounds deserve brief comment. In compound $\mathbf{1 8}$ ring $\mathrm{C}$ conserves the ${ }^{4} C_{1}$ chair conformation of its predecessor and the 1,3-dioxocane ring adopts a restricted boatchair conformation as confirmed by NOESY correlations between the hydrogens at C-4a and H-2', H-3', H-3, and $\mathrm{H}-5$. A similar conformation has been already observed for the 1,3,5-trioxocane ring present in $\mathbf{1 1}^{[3,4]}$ The trans-fused dioxa[4.4.0]bicyclic system in compound $\mathbf{1 9}$ is conformationally locked and ${ }^{4} C_{1}$ chairs are observed for rings $\mathrm{A}$ and B. The configuration of the spirocenter is $S$ and ring $\mathrm{C}$ adopts also a ${ }^{4} C_{1}$ chair conformation. This is confirmed by the coupling constants and the selected NOESY correlations between the axial $\mathrm{H}-4 \mathrm{a}$ and different hydrogens and methoxy groups of the adjacent rings, as shown in Fig- 
ure 1. $^{[15]}$ The nucleophilic intramolecular spirocyclization operates seemingly under thermodynamic control and the most stable conformation is formed in which each ring oxygen is axial with respect to the adjacent ring, thereby gaining maximum stability from the anomeric effect. ${ }^{[16]}$

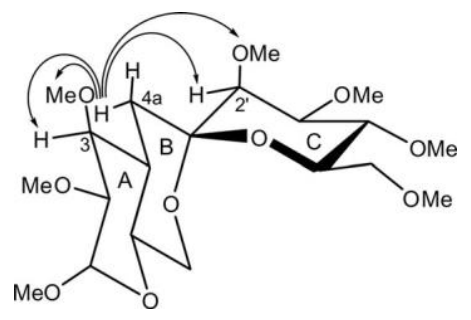

Figure 1. Proposed conformation and selected NOE correlations for spiroacetal 19.

The ${ }^{1} \mathrm{H}$ NMR spectrum of $\mathbf{2 0}$ showed for the H-4a doublet an equatorial-axial coupling $\left({ }^{3} \mathrm{~J}(\mathrm{H} 4 \mathrm{a}, \mathrm{H} 4)=2.8 \mathrm{~Hz}\right.$, calcd. $3.2 \mathrm{~Hz}),{ }^{[17]}$ confirming an $S$ axial stereochemistry for the iodine atom beyond any doubt.

Although compound $\mathbf{2 1}$ could not be isolated in totally pure form, its structure has been tentatively assigned on the basis of ${ }^{1} \mathrm{H}$ and ${ }^{13} \mathrm{C}$ NMR spectroscopic evidence. An interesting feature of this structure is the quaternary hemiacetal carbon at C-2' $(\delta=101.1 \mathrm{ppm})$ which has been assigned by HMBC and HSQC experiments. The observed coupling pattern for the system $\mathrm{H}-1^{\prime}-\mathrm{H}-4 \mathrm{a}-\mathrm{H}-4$ $\left[{ }^{3} J\left(\mathrm{H} 4 \mathrm{a}, \mathrm{H} 1^{\prime}\right)=9.5\right.$ and $\left.{ }^{3} J(\mathrm{H} 4 \mathrm{a}, \mathrm{H} 4)=4.4 \mathrm{~Hz}\right]$ is in good agreement with the calculated one [calcd. $\left({ }^{3} J\left(\mathrm{H} 4 \mathrm{a}, \mathrm{H} 1^{\prime}\right)=\right.$ 10.0 and $\left.\left.{ }^{3} J(\mathrm{H} 4 \mathrm{a}, \mathrm{H} 4)=5.6 \mathrm{~Hz}\right)\right]^{[17]}$ and consistent with an $S \beta$-configuration for the iodine atom.

The reduction of phthalimide $\mathbf{2 2}$ by treatment with $n \mathrm{Bu}_{3} \mathrm{SnH} / \mathrm{AIBN}$ led preferentially to the C-1' inverted alcohol 24 together with a smaller amount of the alcohol inverted at C-5' 23 (Scheme 5). The recovery starting alcohol 2 (40\%) was formed probably by four different processes: stannane quenching with retention at $\mathrm{C}-1$ ' and $\mathrm{C}$ $5^{\prime}$, reduction of the $\mathrm{C}-4 \mathrm{a}$-radical and reduction of the 6-Oalkoxyl radical. Repetition of the experiment with $n \mathrm{Bu}_{3} \mathrm{SnD} / \mathrm{AIBN}$ confirmed this supposition, the isotopic distribution of product 27 measured by MS and ${ }^{1} \mathrm{H}$ NMR $\left({ }^{1} \mathrm{H} /{ }^{2} \mathrm{H}-1^{1} / 2 \mathrm{H}-5^{1} / 2 \mathrm{H}-4 \mathrm{a}, 54: 11: 11: 24\right)$ led to the calculated HATs and percentage of alkoxyl radical reduction shown in Table 2 (entry 4).

Taking into account that in these reductive experiments there is no possibility of double abstraction processes, results are qualitatively similar to those obtained with the HAT oxidative approach (Table 2, entry 3).

In striking contrast to the HAT reaction of $O$-disaccharide 1 where 1,8-hydrogen transfer was observed exclusively, in $C$-disaccharide 2 the process is less regioselective and reactions promoted by 1,5-, 1,6-, 1,7-, and 1,8-HAT to the 6$O$-alkoxyl radical can compete (Table 2, entries $1-4$ ).

At this stage, it is interesting to compare these results with those we obtained from simpler $\alpha-\square$-mannopyranosyl glycoside models $\mathbf{2 8}$ and $\mathbf{3 1}{ }^{[18]}$ and that have been published previously as a preliminary communication (Scheme 6 and Scheme 7). ${ }^{[19]}$ In these cases, the aglycon was a simple $n$ -

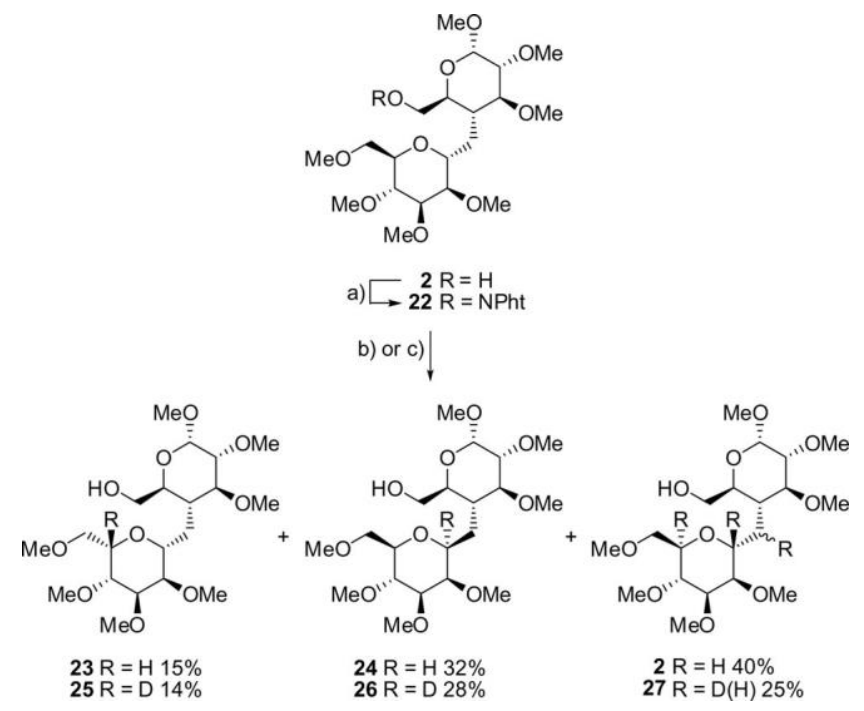

Scheme 5. Reductive HAT of $\alpha-\square-M a n p-(1<4 a)-4 a-c a r b a-\beta-\square-$ Glc $p$ (2); a) DEAD (2.5 equiv.), $N$-hydroxyphthalimide (2 equiv.),

$\mathrm{PPh}_{3}$ (2 equiv.), THF, $0{ }^{\circ} \mathrm{C}, 2.5 \mathrm{~h}, 67 \%$; b) $n \mathrm{Bu} 3 \mathrm{SnH}$ (2 equiv.), AIBN (0.2 equiv.), $\mathrm{PhH}$, reflux, $2 \mathrm{~h}$; c) $n \mathrm{Bu}_{3} \mathrm{SnD}$ (2 equiv.), AIBN (0.2 equiv.), $\mathrm{PhH}$, reflux, 3 h. Isotopic distribution of $27:{ }^{1} \mathrm{H} /{ }^{2} \mathrm{H}-1 /$ ${ }^{2} \mathrm{H}-5^{1 / 2} \mathrm{H}-4 \mathrm{a}, 54: 11: 11: 24$.

alkyl alcohol tether of five atoms, with the least possible conformational constraints, to avoid, as much as possible, destabilizing steric interactions in the HAT transition state.

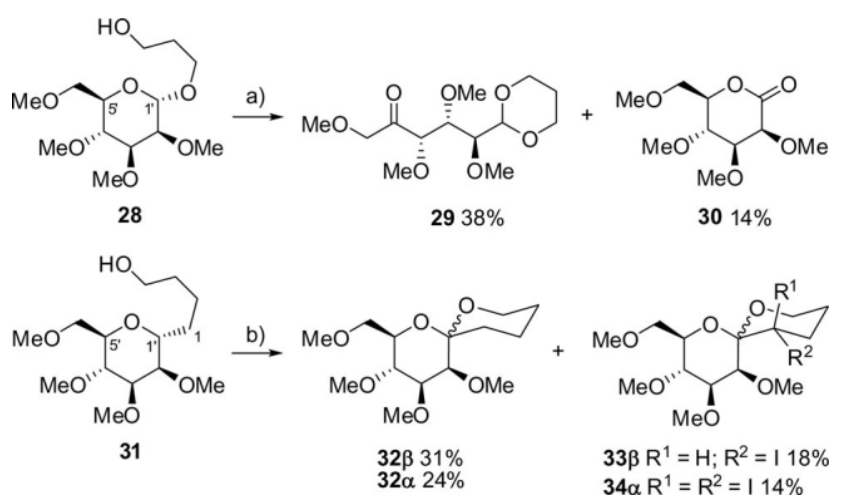

Scheme 6. Oxidative HAT of $O$ - and $C$-glycosides 28 and 31; a) DIB (3.4 equiv.), iodine (2 equiv.), $\mathrm{CH}_{2} \mathrm{Cl}_{2}, h v$, r.t., 8 h; b) DIB (1.7 equiv.), iodine (1 equiv.), $\mathrm{CH}_{2} \mathrm{Cl}_{2}$, $h v$, r.t., $1 \mathrm{~h}$.

The oxidative HAT reaction of $O$-glycoside 28 was accomplished with the $\mathrm{DIB} / \mathrm{I}_{2}$ system to afford two compounds 29 and 30 (Scheme 6). The major product was the ketone 29 resulting from initial intramolecular 1,8-HAT of the 5'-hydrogen followed by acid-catalyzed rearrangement. ${ }^{[20]}$ The minor product was the known lactone $\mathbf{3 0}^{[21]}$ evidently formed by 1,6-HAT of the H-1' and subsequent acid hydrolysis of the spiro ortho ester intermediate (vide infra). The corresponding $C$-glycoside $\mathbf{3 1}$ did not lead to products of 1,8 -HAT but instead gave principally a mixture of isomeric spiroacetals $\mathbf{3 2} \boldsymbol{\alpha}$ and $\mathbf{3 2} \boldsymbol{\beta}$ through 1,6-HAT (Scheme 6). The formation of minor iodine compounds 33 and $\mathbf{3 4}$ probably involved a single or double abstraction at 


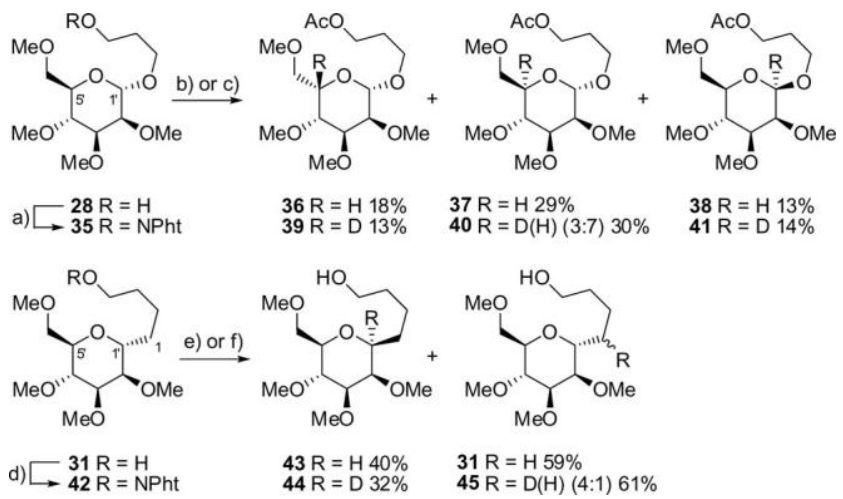

Scheme 7. Reductive HAT of $O$ - and $C$-glycosides 28 and 31 ; a) DEAD (3 equiv.), $N$-hydroxyphthalimide ( 3 equiv.), $\mathrm{PPh}_{3}$ (3 equiv.), THF, $0{ }^{\circ} \mathrm{C}, 1 \mathrm{~h}, 83 \%$; b) $n \mathrm{Bu}_{3} \mathrm{SnH}$ (2 equiv.), AIBN $(0.2$ equiv.), $\mathrm{PhH}$, reflux, $3 \mathrm{~h}$; then $\mathrm{Ac}_{2} \mathrm{O}$, Py, r.t., $4 \mathrm{~h}$; c) $n \mathrm{Bu}_{3} \mathrm{SnD}$ (2 equiv.), $\mathrm{AIBN}$ (0.2 equiv.), $\mathrm{PhH}$, reflux, $4.75 \mathrm{~h}$; then $\mathrm{Ac}_{2} \mathrm{O}$, $\mathrm{Py}$, r.t., $4 \mathrm{~h}$; d) DEAD (2.5 equiv.), $N$-hydroxyphthalimide (2.5 equiv.), $\mathrm{PPh}_{3}$ (2.5 equiv.), THF, $0{ }^{\circ} \mathrm{C}, 2 \mathrm{~h}, 74 \%$; e) $n \mathrm{Bu}_{3} \mathrm{SnH}$ (1 equiv.), AIBN (0.1 equiv.), $\mathrm{PhH}$, reflux, $1 \mathrm{~h}$; f) $n \mathrm{Bu}_{3} \mathrm{SnD}$ ( 1 equiv.), AIBN (0.1 equiv.), $\mathrm{PhH}$, reflux, $1 \mathrm{~h}$.

C-1 (1,5-HAT) and subsequent radical quenching by iodine atoms before the abstraction and ionic spirocyclization at C-1' could take place (Table 2, entries 5 and 7).

The results of the reductive HAT reaction for these models are outlined in Scheme 7. Under reductive conditions the phthalimide $\mathbf{3 5}$ was transformed into three compounds that were characterized after acetylation to facilitate the chromatographic separation: ${ }^{[22]} \beta-\square$-gulopyranoside derivative 36 formed by the 1,8-HAT process and inversion of configuration at C-5' (18\%), $\beta$ - $\square$-mannopyranoside derivative 38 which, evidently, arises from 1,6-HAT, inversion of the radical at $\mathrm{C}-1$ ', and axial quenching by the stannane $(13 \%),{ }^{[23]}$ and compound 37 with the starting $\alpha$ - $\square$-mannopyranoside configuration (29\%). Analogously to the disaccharide 15, compound 37 may be formed by three different mechanisms: abstraction and retention of configuration at C-5' or at C-1' or simply by a failure of the hydrogen abstraction process and a reduction of the $O$-radical prior to the abstraction reaction, or by a mixture of these. Repetition of the reduction of the phthalimide 35 with $n \mathrm{Bu}_{3} \mathrm{SnD}$ shed some more light on the mechanism. Analysis of the isotopic distribution in compounds 39 and 41 showed a complete substitution by deuterium at C-5' and C-1', respectively. On the other hand, only $30 \%$ of deuterium labeling was found at $\mathrm{C}^{-5}$ ' in compound 40; no appreciable labeling at $\mathrm{C}-1$ ' could be observed under the detection limits of the ${ }^{1} \mathrm{H}$ and ${ }^{13} \mathrm{C}$ NMR spectroscopy (Table 2, entry 6). We therefore concluded that in compound $\mathbf{4 0}$ all the deuterium comes only from abstraction-retention at C-5' and that the reduction of the $O$-radical was responsible for the unlabeled molecules.

Several other conclusions can be drawn from the results presented above. For this $O$-glycoside the abstraction at C5', through an apparently less stable nine-membered TS compete favourably with the abstraction at C-1' through a seven-membered TS (C-5':C-1' abstraction ratio of $6: 4)$.
Interestingly, the abstraction at C-5' proceeded with an inversion-retention ratio of $6: 4$, while a complete inversion of configuration is observed during the abstraction at C-1'. ${ }^{[23]}$ The results obtained for the reductive HAT reaction of the $C$-glycoside $\mathbf{4 2}$ are also illustrated in Scheme 7. NMR spectral analysis of compounds $\mathbf{4 3}$ and $\mathbf{3 1}$ and of their deu-

terated counterparts 44 and $\mathbf{4 5}$ revealed that the reaction proceeded by hydrogen abstraction at $\mathrm{C}-1$ ' and $\mathrm{C}-1$, but no abstraction at C-5' could be detected (Table 2, entry 8). As may be expected, the abstraction at $\mathrm{C}-1$ is, in this $C$-glycoside, a competitive reaction since it presumably proceeds via a six-membered TS. The inversion of configuration at C-1'

observed in compound $\mathbf{4 3}$ is in good agreement with the previously reported stabilization of the $\alpha$-radical by the socalled radical anomeric effect. ${ }^{[24]}$

The isomeric alcohol 43, where the four-carbon tether at $\mathrm{C}-1$ ' is now in $\beta$-equatorial position, offered the opportunity to clarify the 1,5- vs. 1,6-competitive HAT observed. As shown in Scheme 8, the alkoxyl radical generated from 43 under oxidative or reductive conditions abstracted exclusively the $\alpha$-axial hydrogen at $\mathrm{C}$ - 1 ' in high yield. Under reductive conditions total retention of configuration was observed (96\% of starting alcohol $\mathbf{4 3}$ was obtained) but the $n \mathrm{Bu}_{3} \mathrm{SnD} / \mathrm{AIBN}$ experiment confirmed the quantitative abstraction (100\% of deuterium incorporation was found in 44). Interestingly, in this $\beta$-equatorial stereoisomer no detectable amount of products from abstraction at C-1 was observed. This may be rationalized by stereoelectronic effects that have a strong influence on the rate of hydrogen transfer. ${ }^{[25]}$ The axial hydrogen at C-1' in $\mathbf{4 3}$ is abstracted very much faster than the equatorial one of $\mathbf{3 1}$ and under these circumstances the 1,5-HAT reaction can not compete.

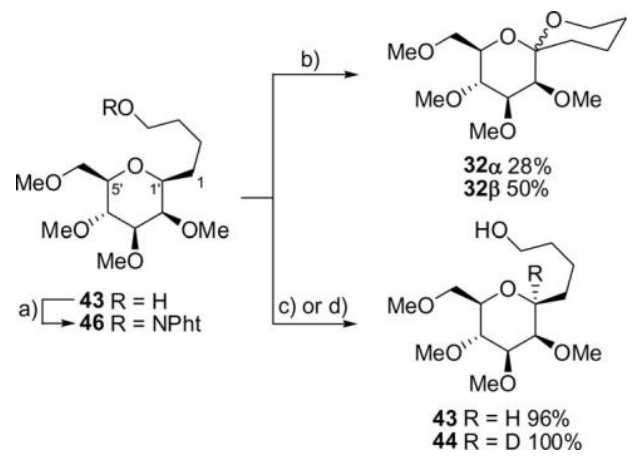

Scheme 8 . HAT of a $C$-Glycoside derived from $\square$-glycero- $\square$-galactodecitol (43); a) DEAD (2.5 equiv.), $N$-hydroxyphthalimide (2.5 equiv.), $\mathrm{PPh}_{3}$ (2.5 equiv.), THF, $0{ }^{\circ} \mathrm{C}, 2 \mathrm{~h}, 79 \%$; b) DIB (1.7 equiv.), iodine (1 equiv.), $\mathrm{CH}_{2} \mathrm{Cl}_{2}$, hv, r.t., 0.5 h; c) $n \mathrm{Bu}_{3} \mathrm{SnH}$ ( 1 equiv.), $\mathrm{AIBN}\left(0.1\right.$ equiv.), $\mathrm{PhH}$, reflux, $1 \mathrm{~h}$; d) $n \mathrm{Bu}_{3} \mathrm{SnD}$ (1.5 equiv.), $\mathrm{AIBN}$ (0.2 equiv.), $\mathrm{PhH}$, reflux, $1.5 \mathrm{~h}$.

\section{Conclusions}

As far as we know, no example of an intramolecular HAT reaction able to discriminate between the hydrogens at $\mathrm{C}-5$ ' and $\mathrm{C}-1$ ' of $\alpha-\square-O$-glycopyranosyl compounds has been reported to date. ${ }^{[26]}$ 
In the case of the simpler $C$-glycoside $\mathbf{3 1}$ and on the basis of electronic factors exclusively, the abstraction of the axial H-5' should be favoured over the equatorial H-1'. However, the reaction gave the opposite result: the abstraction occurred exclusively at $\mathrm{H}-\mathrm{1}^{\prime}$ (disregarding the abstraction at C-4a, 1,5-HAT) and no products coming from abstraction at H-5' could be detected. These results are in agreement with previous studies from our laboratory and others during the alkoxyl radical cyclization of different $C$-glycopyranosides with a similar $n$-alkyl alcohol tether of five atoms. ${ }^{[27]}$ Contrarily, for the $O$-glycoside 28 the axial H-5' was abstracted preferentially.

We believe that the expected greater conformational flexibility of the glycosidic bond in the $C$-glycoside is responsible for the observed results. ${ }^{[2]}$ This permits the electrophilic alkoxyl radical to abstract the electronically disfavoured equatorial H-1' through a more stable seven-membered TS. Due to a more restricted conformation of the $O$-glycosides the HAT reaction proceeded preferentially by abstraction of the favoured axial $\mathrm{H}-5^{\prime}$ via a less stable nine-membered TS.

These results are in qualitative agreement with those obtained for the disaccharides $\mathbf{1}$ and $\mathbf{2}$. In the $O$-disaccharide 1 the 6- $O$-yl radical triggered a 1,8 -HAT reaction by exclusive abstraction of the $\mathrm{H}-5$ ' with the glycosidic bond in the more stable exo-syn conformation. On the contrary, in $C$ disaccharide 2 products coming from 1,5-, 1,6-, 1,7-, and 1,8-HAT processes were isolated, with the spiroacetal $\mathbf{1 9}$, from a 1,6-HAT abstraction of the H-1', as the major product.

Among the models described in this work two extreme situations can be observed: in $O$-disaccharide 1 with maximum steric and stereoelectronic effects, the abstraction occurs exclusively at $\mathrm{H}-5^{\prime}$. On the other hand, in the simpler $C$-glycoside 31, where these effects are minimal and in consequence the glycosidic bond possesses higher flexibility, the abstraction is exclusively at H-1'. We believe that these experiments provided chemical evidence for the conformational differences between $C$ - and $O$-disaccharides, at least in these manno-systems.

\section{Experimental Section}

General Methods: Melting points were determined with a hot-stage apparatus. Optical rotations were measured at the sodium line at ambient temperature in $\mathrm{CHCl}_{3}$ solutions. IR spectra were recorded in film. NMR spectra were determined at 500 or $400 \mathrm{MHz}$ for ${ }^{1} \mathrm{H}$ and 125.7 or $100.6 \mathrm{MHz}$ for ${ }^{13} \mathrm{C}$ in $\mathrm{CDCl}$ or $\mathrm{C} \mathrm{D}$ as specified, in the presence of TMS as internal standard. For convenience, the atom-numbering system used throughout this section for the NMR assignment corresponds to that depicted in the corresponding Scheme although a IUPAC systematic nomeclature have been used. Mass spectra were recorded by using $\mathrm{EI}$ or $\mathrm{ESI}^{+}$as specified in each case. Merck silica gel 60 PF $(0.063-0.2 \mathrm{~mm})$ was used for column chromatography. Circular layers of $1 \mathrm{~mm}$ of Merck silica gel $60 \mathrm{PF}_{254}$ were used on a Chromatotron for centrifugally assisted chromatography. Commercially available reagents and solvents were analytical grade or were purified by standard procedures prior to use. All reactions involving air-or moisture-sensitive materials were carried out under a nitrogen atmosphere. The spray reagents for TLC analysis were conducted with $0.5 \%$ vanillin in $\mathrm{H}_{2} \mathrm{SO}_{4} /$ $\mathrm{EtOH}(4: 1)$ and further heating until development of color.

Oxidative HAT of Methyl 2,3,4,6-Tetra- $O$-methyl- $\alpha$-D-mannopyranosyl-(1<4)-2,3-di- $O$-methyl- $\alpha$-D-glucopyranoside (1): A solution of $1(100 \mathrm{mg}, 0.227 \mathrm{mmol})$ in dry $\mathrm{CH}_{2} \mathrm{Cl}_{2}(8.5 \mathrm{~mL})$ containing iodine $(58 \mathrm{mg}, 0.227 \mathrm{mmol})$ and DIB (124 mg, $0.385 \mathrm{mmol})$ in a flask protected from light with aluminium foil was stirred at reflux temperature under nitrogen for $2 \mathrm{~h}$. After this time another portion of DIB (62 mg, $0.192 \mathrm{mmol}$ ) was added and heating at reflux was continued for an additional $1 \mathrm{~h}$. The reaction mixture was then poured into $10 \%$ aqueous $\mathrm{Na}_{2} \mathrm{~S}_{2} \mathrm{O}_{3}$ and extracted with $\mathrm{CH}_{2} \mathrm{Cl}_{2}$. The organic extracts were dried with $\mathrm{Na}_{2} \mathrm{SO}_{4}$ and concentrated under reduced pressure. The residue was purified by chromatotron chromatography (hexanes/EtOAc, 2:8) to give in order of elution methyl 5',6-anhydro-2',3',4',6'-tetra- $O$-methyl- $\alpha-\square$-lyxo-hexos-5'ulopyranosyl-(1<4)-2,3-di- $O$-methyl- $\alpha$ - $\square$-mannopyranoside (11) (56 mg, $0.128 \mathrm{mmol}, 56 \%$ ), methyl (5'R)-5-O-acetyl-2,3,4,6-tetra$O$-methyl- $\alpha$ - $\square$-ribo-hexos-5-ulopyranosyl-(1<4)-2,3-di- $O$-methyl- $\alpha$ $\square$-glucopyranoside (12及) (25.6 $\mathrm{mg}, 0.051 \mathrm{mmol}, 23 \%$ ) and methyl (5'S)-5- $O$-acetyl-2,3,4,6-tetra- $O$-methyl- $\alpha-\square$-lyxo-hexos-5ulopyranosyl-(1<4)-2,3-di- $O$-methyl- $\alpha$ - $\square$-glucopyranoside (19 mg, $0.038 \mathrm{mmol}, 17 \%$ ), as colourless oils.

Compound 11: $[\alpha]_{\mathrm{D}}=+84.7\left(c=0.170\right.$, in $\left.\mathrm{CHCl}_{3}\right) .{ }^{1} \mathrm{H}$ NMR $\quad(400$ $\left.\mathrm{MHz}, \mathrm{C}_{6} \mathrm{D}_{6}\right): \delta=3.02(\mathrm{~s}, 3 \mathrm{H}, \mathrm{OMe}), 3.11(\mathrm{dd}, J=3.4,9.3 \mathrm{~Hz}$,

$1 \mathrm{H}, 2-\mathrm{H}), 3.19$ (s, $3 \mathrm{H}, \mathrm{OMe}), 3.21$ (s, $3 \mathrm{H}, \mathrm{OMe}), 3.27$ (s, $3 \mathrm{H}$, $\mathrm{OMe}), 3.36$ (s, $3 \mathrm{H}, \mathrm{OMe}), 3.42\left(\mathrm{~d}, J=10.3 \mathrm{~Hz}, 1 \mathrm{H}, 6^{\prime}-\mathrm{H}_{\mathrm{a}}\right), 3.55$ (s, $3 \mathrm{H}, \mathrm{OMe}), 3.63(\mathrm{~s}, 3 \mathrm{H}, \mathrm{OMe}), 3.67\left(\mathrm{~d}, J=10.3 \mathrm{~Hz}, 1 \mathrm{H}, 6^{\prime}-\right.$ $\mathrm{H}_{\mathrm{b}}$ ), 3.76-3.79 (m, 3 H, 4-H, 3-H, 2'-H), 3.83 (m, $\left.1 \mathrm{H}, 5-\mathrm{H}\right), 3.90$ $\left(\mathrm{dd}, J=3.7,11.6 \mathrm{~Hz}, 1 \mathrm{H}, 6-\mathrm{H}_{\mathrm{a}}\right), 4.10(\mathrm{dd}, J=9.8,11.4 \mathrm{~Hz}, 1 \mathrm{H}$, 6- $\left.\mathrm{H}_{\mathrm{b}}\right), 4.24\left(\mathrm{dd}, J=2.9,10.3 \mathrm{~Hz}, 1 \mathrm{H}, 3^{\prime}-\mathrm{H}\right), 4.35(\mathrm{~d}, J=10.3 \mathrm{~Hz}$, $\left.1 \mathrm{H}, 4^{\prime}-\mathrm{H}\right), 4.58(\mathrm{~d}, J=3.4 \mathrm{~Hz}, 1 \mathrm{H}, 1-\mathrm{H}), 5.31(\mathrm{~d}, J=1.6 \mathrm{~Hz}, 1$ $\left.\mathrm{H}, 1^{\prime}-\mathrm{H}\right)$ ppm. ${ }^{13} \mathrm{C}$ NMR $\left(100.6 \mathrm{MHz}, \mathrm{C}_{6} \mathrm{D}_{6}\right): \delta=54.8\left(\mathrm{CH}_{3}\right.$, $\mathrm{OMe}), 58.0\left(\mathrm{CH}_{3}, \mathrm{OMe}\right), 58.1\left(\mathrm{CH}_{3}, \mathrm{OMe}\right), 59.1\left(\mathrm{CH}_{3}, \mathrm{OMe}\right), 59.3$ $\left(\mathrm{CH}_{3}, \mathrm{OMe}\right), 61.05\left(\mathrm{CH}_{3}, \mathrm{OMe}\right), 61.08\left(\mathrm{CH}_{3}, \mathrm{OMe}\right), 65.2\left(\mathrm{CH}_{2}\right.$, C-6), $67.1(\mathrm{CH}, \mathrm{C}-5) 72.9\left(\mathrm{CH}_{2}, \mathrm{C}-6\right.$ ') $77.5(\mathrm{CH}, \mathrm{C}-4$ ') $78.3(\mathrm{CH}$, C-3'), 78.7 (CH, C-2'), $80.0(\mathrm{CH}, \mathrm{C}-4), 81.5(\mathrm{CH}, \mathrm{C}-3), 82.4(\mathrm{CH}$, C-2), 97.9 ( $\mathrm{CH}, \mathrm{C}-1), 99.4\left(\mathrm{CH}, \mathrm{C}^{-1} \mathbf{1}^{\prime}\right) 103.0$ (C, C-5') ppm. IR (film): $\tilde{v}=2931,2828,1101,1064 \mathrm{~cm}^{-1}$. MS $(70 \mathrm{eV}, \mathrm{EI}): \mathrm{m} / \mathrm{z}(\%)=$ $438(<1)[\mathrm{M}]^{+}, 408(<1), 365(43), 233$ (77), 75 (100). HRMS (EI): $m / z$ : calcd. for $\mathrm{C}_{19} \mathrm{H}_{34} \mathrm{O}_{11}: 438.2101[\mathrm{M}]^{+}$; found 438.2102. $\mathrm{C}_{19} \mathrm{H}_{34} \mathrm{O}_{11}$ (438.47): calcd. C 52.05, H 7.82; found C 52.35, H 7.49.

Compound $12 \alpha$ (mixture with compound 12ß): ${ }^{1} \mathrm{H}$ NMR $(400 \mathrm{MHz}$, $\left.\mathrm{C}_{6} \mathrm{D}_{6}\right): \delta=1.84$ (s, $\left.3 \mathrm{H}, \mathrm{OAc}\right), 3.06(\mathrm{~m}, 1 \mathrm{H}, 2-\mathrm{H}), 3.07$ (s, $3 \mathrm{H}$, $\mathrm{OMe}$ ), 3.16 (s, $3 \mathrm{H}, \mathrm{OMe}$ ), 3.20 (s, $3 \mathrm{H}, \mathrm{OMe}$ ), 3.32 (s, $3 \mathrm{H}, \mathrm{OMe}$ ), 3.37 (s, 3 H, OMe), 3.57-3.66 (m, 3 H, 5-H, 3-H, 2'-H), 3.59 (s, 3 $\mathrm{H}, \mathrm{OMe}), 3.61$ (s, 3 H, OMe), 3.95-3.99 (m, $\left.2 \mathrm{H}, 4-\mathrm{H}, 6-\mathrm{H}_{\mathrm{a}}\right), 3.98$ $\left(\mathrm{d}, J=10.6 \mathrm{~Hz}, 1 \mathrm{H}, 6^{\prime}-\mathrm{H}_{\mathrm{a}}\right), 4.12\left(\mathrm{dd}, J=2.1,9.8 \mathrm{~Hz}, 1 \mathrm{H}, 3^{\prime}-\mathrm{H}\right)$, $4.30\left(\mathrm{~m}, 1 \mathrm{H}, 6-\mathrm{H}_{\mathrm{b}}\right), 4.40\left(\mathrm{~d}, J=9.5 \mathrm{~Hz}, 1 \mathrm{H}, 4^{\prime}-\mathrm{H}\right), 4.56(\mathrm{~d}, J=$ $3.4 \mathrm{~Hz}, 1 \mathrm{H}, 1-\mathrm{H}), 4.60$ (d, $\left.J=10.6 \mathrm{~Hz}, 1 \mathrm{H}, 6^{\prime}-\mathrm{H}_{\mathrm{b}}\right), 5.33$ (d, $J=$ $\left.2.4 \mathrm{~Hz}, 1 \mathrm{H}, 11^{\prime}-\mathrm{H}\right) \mathrm{ppm} .{ }^{13} \mathrm{C}$ NMR $\left(100.6 \mathrm{MHz}, \mathrm{C}_{6} \mathrm{D}_{6}\right): \delta=22.4$ $\left(\mathrm{CH}_{3}, \mathrm{OAc}\right), 54.8\left(\mathrm{CH}_{3}, \mathrm{OMe}\right), 57.81\left(\mathrm{CH}_{3}, \mathrm{OMe}\right), 57.84\left(\mathrm{CH}_{3}\right.$, $\mathrm{OMe}), 59.1\left(\mathrm{CH}_{3}, \mathrm{OMe}\right), 60.8\left(\mathrm{CH}_{3} \mathrm{OMe}\right), 61.4\left(\mathrm{CH}_{3} \mathrm{OMe}\right), 61.5$ $\left(\mathrm{CH}_{3}, \mathrm{OMe}\right), 61.5\left(\mathrm{CH}_{2}, \mathrm{C}-6\right), 71.8(\mathrm{CH}, \mathrm{C}-3) 72.8\left(\mathrm{CH}_{2}, \mathrm{C}-6\right)$, 76.5 (CH, C-4'), 77.5 (CH, C-3'), $78.0(\mathrm{CH}, \mathrm{C}-4), 79.1$ (CH, C-5), $82.3\left(\mathrm{CH}, \mathrm{C}-2^{\prime}\right), 82.8(\mathrm{CH}, \mathrm{C}-2), 97.6(\mathrm{CH}, \mathrm{C}-1), 103.1\left(\mathrm{CH}, \mathrm{C}-1^{\prime}\right)$, 105.7 (C, C-5') 169.4 (C, OAc) ppm. MS (70 eV, EI): $\mathrm{m} / \mathrm{z}(\%)=$ 438 (1) $\left[M-\mathrm{CH}_{3} \mathrm{CO}_{2} \mathrm{H}\right]^{+}, 421$ (1), 393 (2), 145 (50), 101 (95), 88 (100). HRMS (EI): $\mathrm{m} / z$ : calcd. for $\mathrm{C}_{19} \mathrm{H}_{34} \mathrm{O}_{11}$ : $438.2101[M-$ $\left.\mathrm{CH}_{3} \mathrm{CO}_{2} \mathrm{H}\right]^{+}$; found 438.2107 .

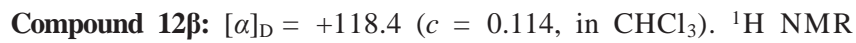
(500 MHz, $\mathrm{C}_{6} \mathrm{D}_{6}$ ): $\delta=1.76$ (s, $3 \mathrm{H}, \mathrm{OAc}$ ), 3.05 (s, $\left.3 \mathrm{H}, \mathrm{OMe}\right), 3.10$ 
(s, $3 \mathrm{H}, \mathrm{OMe}$ ), 3.125 (m, $1 \mathrm{H}, 2-\mathrm{H}), 3.128$ (s, $3 \mathrm{H}, \mathrm{OMe}), 3.14$ (s, $3 \mathrm{H}, \mathrm{OMe}$ ), 3.27 (s, $3 \mathrm{H}, \mathrm{OMe}), 3.36$ (s, $3 \mathrm{H}, \mathrm{OMe}), 3.55$ (dd, $J=$ $\left.3.4,8.4 \mathrm{~Hz}, 1 \mathrm{H}, 2^{\prime}-\mathrm{H}\right), 3.65$ (dd, $\left.J=3.2,3.2 \mathrm{~Hz}, 1 \mathrm{H}, 3^{\prime}-\mathrm{H}\right), 3.71$ (s, $3 \mathrm{H}, \mathrm{OMe}$ ), 3.75 (ddd, $J=2.4,2.4,10.2 \mathrm{~Hz}, 1 \mathrm{H}, 5-\mathrm{H}), 3.91$ (dd, $J=9.2,9.2 \mathrm{~Hz}, 1 \mathrm{H}, 3-\mathrm{H}), 3.97$ (d, $J=3.1 \mathrm{~Hz}, 1 \mathrm{H}, 4^{\prime}-\mathrm{H}$ ), $4.05\left(\mathrm{dd}, J=2.5,12.3 \mathrm{~Hz}, 1 \mathrm{H}, 6-\mathrm{H}_{\mathrm{a}}\right), 4.12\left(\mathrm{~s}, 2 \mathrm{H}, 6{ }^{\prime}-\mathrm{H}_{2}\right), 4.18$ $(\mathrm{dd}, J=9.0,10.1 \mathrm{~Hz}, 1 \mathrm{H}, 4-\mathrm{H}), 4.42(\mathrm{dd}, J=2.5,12.6 \mathrm{~Hz}, 1 \mathrm{H}$, $\left.6-\mathrm{H}_{\mathrm{b}}\right), 4.67(\mathrm{~d}, J=3.6 \mathrm{~Hz}, 1 \mathrm{H}, 1-\mathrm{H}), 5.80\left(\mathrm{~d}, J=8.4 \mathrm{~Hz}, 1 \mathrm{H}, 1^{\prime}-\right.$ H) ppm. ${ }^{13} \mathrm{C} \mathrm{NMR}\left(125.7 \mathrm{MHz}, \mathrm{C}_{6} \mathrm{D}_{6}\right): \delta=21.6\left(\mathrm{CH}_{3}, \mathrm{OAc}\right), 54.7$ $\left(\mathrm{CH}_{3}, \mathrm{OMe}\right), 58.0\left(\mathrm{CH}_{3}, \mathrm{OMe}\right), 58.92\left(\mathrm{CH}_{3}, \mathrm{OMe}\right), 58.96\left(\mathrm{CH}_{3}\right.$, $\mathrm{OMe}), 59.3\left(\mathrm{CH}_{3}, \mathrm{OMe}\right), 59.4\left(\mathrm{CH}_{3}, \mathrm{OMe}\right), 61.1\left(\mathrm{CH}_{3}, \mathrm{OMe}\right), 61.3$ $\left(\mathrm{CH}_{2}, \mathrm{C}-6\right), 71.2\left(\mathrm{CH}_{2}, \mathrm{C}-6 '\right), 71.5(\mathrm{CH}, \mathrm{C}-5), 75.8(\mathrm{CH}, \mathrm{C}-4), 76.5$ (CH, C-4), 78.1 (CH, C-2'), 78.3 (CH, C-3'), 82.6 (CH, C-2), 83.8 (CH, C-3), 98.0 (CH, C-1), 99.2 (CH, C-1'), 104.3 (C, C-5') 169.6 (C, OAc) ppm. IR (film): $\tilde{v}=3534,2935,1738,1370,1245,1103$ $\mathrm{cm}^{-1}$. MS $(70 \mathrm{eV}, \mathrm{EI}): \mathrm{m} / \mathrm{z}(\%)=438(<1)\left[M-\mathrm{CH}_{3} \mathrm{CO}_{2} \mathrm{H}\right]^{+}, 407$ (<1), 365 (7), 278 (19), 145 (34), 101 (84), 88 (100). HRMS (EI): $m / z$ : calcd. for $\mathrm{C}_{19} \mathrm{H}_{34} \mathrm{O}_{11}$ : $438.2101\left[M-\mathrm{CH}_{3} \mathrm{CO}_{2} \mathrm{H}\right]^{+}$; found 438.2091. $\mathrm{C}_{21} \mathrm{H}_{38} \mathrm{O}_{13}$ (498.52): calcd. C 50.59, $\mathrm{H}$ 7.68; found $\mathrm{C}$ 50.55, H 7.47 .

Reductive HAT of Methyl 2,3,4,6-Tetra- $O$-methyl- $\alpha$-D-mannopyranosyl- $(1<4)-2,3$-di- $O$-methyl-6- $O$-phthalimido- $\alpha$-D-glucopyranoside (13). Method $\mathrm{A}\left(n \mathrm{Bu}_{3} \mathrm{SnH} / \mathrm{AIBN}\right)$ : A solution of phthalimide 13 (100 mg, $0.170 \mathrm{mmol})$ in dry benzene $(12 \mathrm{~mL})$ containing $n \mathrm{Bu}_{3} \mathrm{SnH}(68 \mu \mathrm{L}, 0.255 \mathrm{mmol})$ and $\operatorname{AIBN}(5.5 \mathrm{mg}, 0.034 \mathrm{mmol})$ was heated at reflux temperature under nitrogen for $2.5 \mathrm{~h}$. After this time another portion of $n \mathrm{Bu}_{3} \mathrm{SnH}(23 \mu \mathrm{L}, 0.086 \mathrm{mmol})$ and AIBN (5.5 mg, $0.034 \mathrm{mmol}$ ) were added and heating at reflux was continued for an additional $1 \mathrm{~h}$. After cooling at room temperature the mixture was concentrated under reduced pressure. The residue was dissolved in $\mathrm{CH}_{3} \mathrm{CN}$, washed with $n$-hexane and the combined more polar extracts were concentrated under reduced pressure. A solution of the residue in dry pyridine $\left(3 \mathrm{~mL}\right.$ ) containing $\mathrm{Ac}_{2} \mathrm{O}$ (1 $\mathrm{mL}$ ) was stirred at room temperature for $4 \mathrm{~h}$. The mixture was then poured into $10 \%$ aqueous $\mathrm{HCl}$ and extracted with $\mathrm{CH}_{2} \mathrm{Cl}_{2}$. The organic layer was washed with aqueous saturated $\mathrm{NaHCO}_{3}$, dried with $\mathrm{Na}_{2} \mathrm{SO}_{4}$ and concentrated under reduced pressure. The residue was purified by chromatotron chromatography (hexanes/ EtOAc, 4:6) to give in order of elution methyl tetra- $O$-methyl- $\beta$ $\square$ gulopyranosyl-(1< 4)-6- $O$-acetyl-2,3-di- $O$-methyl- $\alpha$ - $\square$-glucopyranoside (14) (38 mg, $0.078 \mathrm{mmol}, 46 \%$ ) and methyl 2,3,4,6tetra- $O$-methyl- $\alpha-\square-$ mannopyranosyl- $(1<4)-6-O$-acetyl-2,3-di- $O$ methyl- $\alpha$ - $\square$-glucopyranoside (15) (14 mg, $0.029 \mathrm{mmol}, 17 \%$ ), both as colourless oils.

Compound 14: $[\alpha]_{\mathrm{D}}=+89.6\left(c=0.240\right.$, in $\left.\mathrm{CHCl}_{3}\right) .{ }^{1} \mathrm{H} \mathrm{NMR} \quad(500$ $\mathrm{MHz}, \mathrm{C}_{6} \mathrm{D}_{6}$ ): $\delta=1.75$ (s, $\left.3 \mathrm{H}, \mathrm{OAc}\right), 3.11$ (s, $3 \mathrm{H}, \mathrm{OMe}$ ), 3.136 (s, $3 \mathrm{H}, \mathrm{OMe}$ ), 3.138 (dd, $J=3.6,8.9 \mathrm{~Hz}, 1 \mathrm{H}, 2-\mathrm{H}), 3.16$ (s, $3 \mathrm{H}$, $\mathrm{OMe}$ ), 3.18 (s, $3 \mathrm{H}, \mathrm{OMe}$ ), 3.28 (dd, $\left.J=1.4,3.6 \mathrm{~Hz}, 1 \mathrm{H}, 4^{\prime}-\mathrm{H}\right)$, 3.29 (s, $3 \mathrm{H}, \mathrm{OMe}), 3.36$ (s, $3 \mathrm{H}, \mathrm{OMe}), 3.46(\mathrm{dd}, J=3.1,8.1 \mathrm{~Hz}$, $\left.1 \mathrm{H}, 2^{\prime}-\mathrm{H}\right), 3.55\left(\mathrm{dd}, J=5.9,9.2 \mathrm{~Hz}, 1 \mathrm{H}, 6^{\prime}-\mathrm{H}_{\mathrm{a}}\right), 3.67(\mathrm{dd}, J=$ 3.6, 3.6 Hz, $\left.1 \mathrm{H}, 3^{\prime}-\mathrm{H}\right), 3.68$ (dd, $\left.J=7.6,9.2 \mathrm{~Hz}, 1 \mathrm{H}, 6^{\prime}-\mathrm{H}_{\mathrm{b}}\right), 3.75$ (s, $3 \mathrm{H}, \mathrm{OMe}$ ), 3.85-3.91 (m, $2 \mathrm{H}, 4-\mathrm{H}, 3-\mathrm{H}), 4.01$ (ddd, $J=1.9$, $6.7,8.9 \mathrm{~Hz}, 1 \mathrm{H}, 5-\mathrm{H}), 4.14$ (ddd, $\left.J=1.4,6.2,7.6 \mathrm{~Hz}, 1 \mathrm{H}, 5^{\prime}-\mathrm{H}\right)$, $4.64\left(\mathrm{dd}, J=6.7,12.3 \mathrm{~Hz}, 1 \mathrm{H}, 6-\mathrm{H}_{\mathrm{a}}\right), 4.65(\mathrm{~d}, J=3.6 \mathrm{~Hz}, 1 \mathrm{H}, 1-$ $\mathrm{H}), 4.83\left(\mathrm{dd}, J=2.2,12.0 \mathrm{~Hz}, 1 \mathrm{H}, 6-\mathrm{H}_{\mathrm{b}}\right), 5.43(\mathrm{~d}, J=8.1 \mathrm{~Hz}, 1$ $\left.\mathrm{H}, 1^{\prime}-\mathrm{H}\right) \mathrm{ppm} .{ }^{13} \mathrm{C} \mathrm{NMR}\left(125.7 \mathrm{MHz}, \mathrm{C}_{6} \mathrm{D}_{6}\right): \delta=20.6\left(\mathrm{CH}_{3}, \mathrm{OAc}\right)$, $54.8\left(\mathrm{CH}_{3}, \mathrm{OMe}\right), 58.1\left(\mathrm{CH}_{3}, \mathrm{OMe}\right), 58.4\left(\mathrm{CH}_{3}, \mathrm{OMe}\right), 58.8\left(\mathrm{CH}_{3}\right.$, $\mathrm{OMe}), 59.3\left(\mathrm{CH}_{3}, \mathrm{OMe}\right), 59.5\left(\mathrm{CH}_{3}, \mathrm{OMe}\right), 61.1\left(\mathrm{CH}_{3}, \mathrm{OMe}\right), 64.6$ $\left(\mathrm{CH}_{2}, \mathrm{C}-6\right), 69.0(\mathrm{CH}, \mathrm{C}-5), 71.160\left(\mathrm{CH}_{2}, \mathrm{C}-6{ }^{\prime}\right), 71.9\left(\mathrm{CH}, \mathrm{C}-5^{\prime}\right)$, $76.3(\mathrm{CH}, \mathrm{C}-4), 77.5\left(\mathrm{CH}, \mathrm{C}-3{ }^{\prime}\right), 77.574\left(\mathrm{CH}, \mathrm{C}-4{ }^{\prime}\right), 80.0(\mathrm{CH}, \mathrm{C}-$ 2'), 82.5 (CH, C-2), 84.0 (CH, C-3), 97.7 (CH, C-1), $101.7(\mathrm{CH}, \mathrm{C}-$ 1'), 170.0 (C, OAc) ppm. IR (film): $\tilde{v}=2906,1739,1243,1048$, $1033 \mathrm{~cm}^{-1}$. MS $(70 \mathrm{eV}, \mathrm{EI}): \mathrm{m} / z(\%)=450(<1)\left[M-\mathrm{CH}_{3} \mathrm{OH}\right]^{+}, 419$
(<1), 307 (48), 247 (100). HRMS (EI): $m / z$ : calcd. for $\mathrm{C}_{20} \mathrm{H}_{34} \mathrm{O}_{11}$ : 450.2101 $\left[M-\mathrm{CH}_{3} \mathrm{OH}\right]^{+}$; found 450.2108. $\mathrm{C}_{21} \mathrm{H}_{38} \mathrm{O}_{12}$ (482.53): calcd. C 52.27, H 7.94; found C 52.28, H 7.91.

Compound 15: $[\alpha]_{\mathrm{D}}=+62.3\left(c=0.960\right.$, in $\left.\mathrm{CHCl}_{3}\right) .{ }^{1} \mathrm{H}$ NMR $(400$ $\left.\mathrm{MHz}, \mathrm{C}_{6} \mathrm{D}_{6}\right): \delta=1.77$ (s, $\left.3 \mathrm{H}, \mathrm{OAc}\right), 3.06(\mathrm{dd}, J=3.4,9.5 \mathrm{~Hz}$,

$1 \mathrm{H}, 2-\mathrm{H}), 3.08$ (s, $3 \mathrm{H}, \mathrm{OMe}), 3.09$ (s, $3 \mathrm{H}, \mathrm{OMe}), 3.27$ (s, $3 \mathrm{H}$, $\mathrm{OMe}$ ), 3.33 (s, $3 \mathrm{H}, \mathrm{OMe}$ ), 3.35 (s, $3 \mathrm{H}, \mathrm{OMe}$ ), 3.48 (s, $3 \mathrm{H}$, OMe), $3.51(\mathrm{~s}, 3 \mathrm{H}, \mathrm{OMe}), 3.69-3.82(\mathrm{~m}, 7 \mathrm{H}), 3.89(\mathrm{dd}, J=9.8,9.8 \mathrm{~Hz}$, $\left.1 \mathrm{H}, 4^{\prime}-\mathrm{H}\right), 4.01$ (ddd, $\left.J=1.9,5.0,9.8 \mathrm{~Hz}, 1 \mathrm{H}, 5^{\prime}-\mathrm{H}\right), 4.39$ (dd, $J$ $\left.=4.8,12.2 \mathrm{~Hz}, 1 \mathrm{H}, 6-\mathrm{H}_{\mathrm{a}}\right), 4.58(\mathrm{~d}, J=3.4 \mathrm{~Hz}, 1 \mathrm{H}, 1-\mathrm{H}), 4.67$ $\left(\mathrm{dd}, J=2.1,11.9 \mathrm{~Hz}, 1 \mathrm{H}, 6-\mathrm{H}_{\mathrm{b}}\right), 5.55\left(\mathrm{~d}, J=1.9 \mathrm{~Hz}, 1 \mathrm{H}, 1^{\prime}-\mathrm{H}\right)$ ppm. ${ }^{13} \mathrm{C}$ NMR (100.6 MHz, $\left.\mathrm{C}_{6} \mathrm{D}_{6}\right): \delta=20.5\left(\mathrm{CH}_{3}, \mathrm{OAc}\right), 54.8$ $\left(\mathrm{CH}_{3}, \mathrm{OMe}\right), 57.3\left(\mathrm{CH}_{3}, \mathrm{OMe}\right), 57.6\left(\mathrm{CH}_{3}, \mathrm{OMe}\right), 58.6\left(\mathrm{CH}_{3}\right.$, $\mathrm{OMe}), 59.1\left(\mathrm{CH}_{3}, \mathrm{OMe}\right), 60.4\left(\mathrm{CH}_{3}, \mathrm{OMe}\right), 60.8\left(\mathrm{CH}_{3}, \mathrm{OMe}\right), 63.9$ $\left(\mathrm{CH}_{2}, \mathrm{C}-6\right), 68.8(\mathrm{CH}, \mathrm{C}-5), 72.4\left(\mathrm{CH}_{2}, \mathrm{C}-6{ }^{\prime}\right), 73.9\left(\mathrm{CH}, \mathrm{C}-5^{\prime}\right), 76.9$ $\left(\mathrm{CH}, \mathrm{C}-4^{\prime}\right), 77.3(\mathrm{CH}, \mathrm{C}-4), 78.3\left(\mathrm{CH}, \mathrm{C}-2^{\prime}\right), 82.0\left(\mathrm{CH}, \mathrm{C}-3{ }^{\prime}\right), 82.6$ (CH, C-2), 83.8 (CH, C-3), 97.4 (CH, C-1), 100.2 (CH, C-1'), 170.0 (C, OAc) ppm. IR (film): $\tilde{v}=2906,1742,1241,1048 \mathrm{~cm}^{-1}$. MS $(70 \mathrm{eV}, \mathrm{EI}): m / z(\%)=419$ (1) $\left[M-\mathrm{CH}_{3} \mathrm{OH}-\mathrm{CH}_{3} \mathrm{O}\right]^{+}, 405$ (1), 307 (100). HRMS (EI): $\mathrm{m} / z$ : calcd. for $\mathrm{C}_{19} \mathrm{H}_{31} \mathrm{O}_{10}: 419.1917[M-$ $\left.\mathrm{CH}_{3} \mathrm{OH}-\mathrm{CH}_{3} \mathrm{O}\right]^{+}$; found 419.1927. $\mathrm{C}_{21} \mathrm{H}_{38} \mathrm{O}_{12}$ (482.53): calcd. $\mathrm{C}$ 52.27, H 7.94; found C 52.25, H 8.03.

Method B ( $\left.n \mathbf{B u}_{3} \mathrm{SnD} / \mathrm{AIBN}\right)$ : A solution of phthalimide $\mathbf{1 3}(82 \mathrm{mg}$, $0.140 \mathrm{mmol})$ in dry benzene $(10 \mathrm{~mL})$ containing $n \mathrm{Bu}_{3} \mathrm{SnD}(56 \mu \mathrm{L}$, $0.210 \mathrm{mmol}$ ) and AIBN (4.6 mg, $0.028 \mathrm{mmol}$ ) was heated at reflux temperature under nitrogen for $2.5 \mathrm{~h}$. After this time another portion of $n \mathrm{Bu}_{3} \mathrm{SnD}(37 \mu \mathrm{L}, 0.138 \mathrm{mmol})$ and $\mathrm{AIBN}(4.6 \mathrm{mg}$, $0.028 \mathrm{mmol}$ ) were added and heating at reflux was continued for an additional $1 \mathrm{~h}$. After cooling at room temperature the mixture was concentrated under reduced pressure. The residue was dissolved in $\mathrm{CH}_{3} \mathrm{CN}$, washed with $n$-hexane and the combined more polar extracts were concentrated under reduced pressure. A solution of the residue in dry pyridine $(3 \mathrm{~mL})$ containing $\mathrm{Ac}_{2} \mathrm{O}(1 \mathrm{~mL})$ was stirred at room temperature for $4 \mathrm{~h}$. The mixture was then poured into $10 \%$ aqueous $\mathrm{HCl}$ and extracted with $\mathrm{CH}_{2} \mathrm{Cl}_{2}$. The organic layer was washed with aqueous saturated $\mathrm{NaHCO}_{3}$, dried with $\mathrm{Na}_{2} \mathrm{SO}_{4}$ and concentrated under reduced pressure. The residue was purified by chromatotron chromatography (hexanes/EtOAc, $4: 6)$ to give in order of elution methyl $2,3,4,6$-tetra- $O$-methyl- $\beta$ - $\square$ $\left(5^{\prime}-{ }^{2} \mathrm{H}\right)$-gulopyranosyl-( $\left.1<4\right)-6$ - $O$-acetyl-2,3-di- $O$-methyl- $\alpha$ - $\square$ glucopyranoside (16) (35 mg, $0.072 \mathrm{mmol}, 52 \%$ ) and methyl 2,3,4,6-tetra- $O$-methyl- $\alpha-\square-\left[5^{\prime}-{ }^{2} \mathrm{H}\right]$ mannopyranosyl-( $\left.1<4\right)-6$ - $O$-acetyl-2,3-di- $O$-methyl- $\alpha$ - $\square$-glucopyranoside (17) $(14 \mathrm{mg}, 0.029 \mathrm{mmol}$, $21 \%,{ }^{1} \mathrm{H} /{ }^{2} \mathrm{H}, 30: 70$ ), as colourless oils.

Compound 16: ${ }^{1} \mathrm{H}$ NMR (500 MHz, $\left.\mathrm{C}_{6} \mathrm{D}_{6}\right): \delta=1.75$ (s, $\left.3 \mathrm{H}, \mathrm{OAc}\right)$, 3.11 (s, $3 \mathrm{H}, \mathrm{OMe}$ ), 3.136 (s, $3 \mathrm{H}, \mathrm{OMe}$ ), 3.137 (dd, $J=3.6,9.2 \mathrm{~Hz}$, $1 \mathrm{H}, 2-\mathrm{H}), 3.16(\mathrm{~s}, 3 \mathrm{H}, \mathrm{OMe}), 3.18(\mathrm{~s}, 3 \mathrm{H}, \mathrm{OMe}), 3.27$ (d, $J=$ $\left.3.6 \mathrm{~Hz}, 1 \mathrm{H}, 4^{\prime}-\mathrm{H}\right), 3.29$ (s, $3 \mathrm{H}, \mathrm{OMe}$ ), 3.36 (s, $3 \mathrm{H}, \mathrm{OMe}$ ), 3.45 $\left(\mathrm{dd}, J=2.8,8.1 \mathrm{~Hz}, 1 \mathrm{H}, 2^{\prime}-\mathrm{H}\right), 3.54\left(\mathrm{~d}, J=9.2 \mathrm{~Hz}, 1 \mathrm{H}, 6^{\prime}-\mathrm{H}_{\mathrm{a}}\right)$, $3.66\left(\mathrm{dd}, J=3.4,3.4 \mathrm{~Hz}, 1 \mathrm{H}, 3^{\prime}-\mathrm{H}\right), 3.68\left(\mathrm{~d}, J=9.2 \mathrm{~Hz}, 1 \mathrm{H}, 6^{\prime}-\right.$ $\mathrm{H}_{\mathrm{b}}$ ), 3.75 (s, 3 H, OMe), 3.85-3.91 (m, $\left.2 \mathrm{H}, 4-\mathrm{H}, 3-\mathrm{H}\right), 4.00$ (ddd, $J=2.2,6.7,8.9 \mathrm{~Hz}, 1 \mathrm{H}, 5-\mathrm{H}), 4.64(\mathrm{dd}, J=6.7,12.0 \mathrm{~Hz}, 1 \mathrm{H}, 6-$ $\left.\mathrm{H}_{\mathrm{a}}\right), 4.65(\mathrm{~d}, J=3.4 \mathrm{~Hz}, 1 \mathrm{H}, 1-\mathrm{H}), 4.83(\mathrm{dd}, J=2.2,11.8 \mathrm{~Hz}, 1$ $\left.\mathrm{H}, 6-\mathrm{H}_{\mathrm{b}}\right), 5.43\left(\mathrm{~d}, J=8.1 \mathrm{~Hz}, 1 \mathrm{H}, 11^{\prime}-\mathrm{H}\right) \mathrm{ppm} .{ }^{13} \mathrm{C} \mathrm{NMR}$ $\left(125.7 \mathrm{MHz}, \mathrm{C}_{6} \mathrm{D}_{6}\right): \delta=20.6\left(\mathrm{CH}_{3}, \mathrm{OAc}\right), 54.8\left(\mathrm{CH}_{3}, \mathrm{OMe}\right), 58.1$ $\left(\mathrm{CH}_{3}, \mathrm{OMe}\right), 58.4\left(\mathrm{CH}_{3}, \mathrm{OMe}\right), 58.8\left(\mathrm{CH}_{3}, \mathrm{OMe}\right), 59.3\left(\mathrm{CH}_{3}\right.$, $\mathrm{OMe}), 59.5\left(\mathrm{CH}_{3}, \mathrm{OMe}\right), 61.1\left(\mathrm{CH}_{3}, \mathrm{OMe}\right), 64.6\left(\mathrm{CH}_{2}, \mathrm{C}-6\right), 69.0$ $(\mathrm{CH}, \mathrm{C}-5), 71.091\left(\mathrm{CH}_{2}, \mathrm{C}-6{ }^{\prime}\right), 76.3(\mathrm{CH}, \mathrm{C}-4), 77.47\left(\mathrm{CH}, \mathrm{C}-3{ }^{\prime}\right)$, $77.522\left(\mathrm{CH}, \mathrm{C}-4^{\prime}\right), 80.0\left(\mathrm{CH}, \mathrm{C}-2^{\prime}\right), 82.5(\mathrm{CH}, \mathrm{C}-2), 84.0(\mathrm{CH}, \mathrm{C}-$ 3), $97.7(\mathrm{CH}, \mathrm{C}-1), 101.6\left(\mathrm{CH}, \mathrm{C}-1^{\prime}\right), 170.0$ (C, OAc) ppm. MS $(70 \mathrm{eV}, \mathrm{EI}): \mathrm{m} / z(\%)=452(<1)\left[M-\mathrm{CH}_{3} \mathrm{O}\right]^{+}, 307$ (43), 247 (70), 88 (100). HRMS (EI): $m / z$ : calcd. for $\mathrm{C}_{20} \mathrm{H}_{34}{ }^{2} \mathrm{HO}_{11}$ : $452.2242[M-$ $\left.\mathrm{CH}_{3} \mathrm{O}\right]^{+}$; found 452.2242 . 
Compound 17: ${ }^{1} \mathrm{H}$ NMR (500 MHz, $\left.\mathrm{C}_{6} \mathrm{D}_{6}\right): \delta=1.77$ (s, $\left.3 \mathrm{H}, \mathrm{OAc}\right)$, $3.06(\mathrm{dd}, J=3.4,9.3 \mathrm{~Hz}, 1 \mathrm{H}, 2-\mathrm{H}), 3.087$ (s, $3 \mathrm{H}, \mathrm{OMe}$ ), 3.090 (s, $3 \mathrm{H}, \mathrm{OMe}$ ), 3.28 (s, $3 \mathrm{H}, \mathrm{OMe}$ ), 3.33 (s, $3 \mathrm{H}, \mathrm{OMe}$ ), 3.35 (s, $3 \mathrm{H}$, OMe), 3.48 (s, $3 \mathrm{H}, \mathrm{OMe}$ ), 3.51 (s, $3 \mathrm{H}, \mathrm{OMe}), 3.70-3.82(\mathrm{~m}, 7 \mathrm{H})$, $3.894\left(\mathrm{~d}, J=9.0 \mathrm{~Hz}, 4^{\prime}-\mathrm{H}-\mathrm{D}\right), 3.897$ (dd, $J=9.3,9.3 \mathrm{~Hz}, 1 \mathrm{H}, 4^{\prime}-$ H), 4.01 (ddd, $\left.J=1.7,5.1,9.9 \mathrm{~Hz}, 1 \mathrm{H}, 5^{\prime}-\mathrm{H}\right), 4.40$ (dd, $J=5.1$, $\left.12.1 \mathrm{~Hz}, 1 \mathrm{H}, 6-\mathrm{H}_{\mathrm{a}}\right), 4.57(\mathrm{~d}, J=3.7 \mathrm{~Hz}, 1 \mathrm{H}, 1-\mathrm{H}), 4.67(\mathrm{dd}, J=$ 2.2, $\left.11.9 \mathrm{~Hz}, 1 \mathrm{H}, 6-\mathrm{H}_{\mathrm{b}}\right), 5.55\left(\mathrm{~d}, J=2.0 \mathrm{~Hz}, 1 \mathrm{H}, 1^{\prime}-\mathrm{H}\right) \mathrm{ppm} .{ }^{13} \mathrm{C}$ NMR (125.7 MHz, $\left.\mathrm{C}_{6} \mathrm{D}_{6}\right): \delta=20.5\left(\mathrm{CH}_{3}, \mathrm{OAc}\right), 54.8\left(\mathrm{CH}_{3}, \mathrm{OMe}\right)$, $57.3\left(\mathrm{CH}_{3}, \mathrm{OMe}\right), 57.6\left(\mathrm{CH}_{3}, \mathrm{OMe}\right), 58.6\left(\mathrm{CH}_{3}, \mathrm{OMe}\right), 59.1\left(\mathrm{CH}_{3}\right.$, $\mathrm{OMe}), 60.4\left(\mathrm{CH}_{3}, \mathrm{OMe}\right), 60.8\left(\mathrm{CH}_{3}, \mathrm{OMe}\right), 63.9\left(\mathrm{CH}_{2}, \mathrm{C}-6\right), 68.8$ (CH, C-5), $72.312\left(\mathrm{CH}_{2}, \mathrm{C}-6\right.$ '-D), $72.376\left(\mathrm{CH}_{2}, \mathrm{C}-6\right.$ '-H), $73.9(\mathrm{CH}$, C-5'-H), 76.894 (CH, C-4'-D), 76.947 (CH, C-4'-H), 77.3 (CH, C4), $78.3\left(\mathrm{CH}, \mathrm{C}-2^{\prime}\right), 81.9\left(\mathrm{CH}, \mathrm{C}-3^{\prime}\right), 82.6(\mathrm{CH}, \mathrm{C}-2), 83.8(\mathrm{CH}, \mathrm{C}-$ 3), $97.4(\mathrm{CH}, \mathrm{C}-1), 100.2\left(\mathrm{CH}, \mathrm{C}-1^{\prime}\right), 170.0(\mathrm{C}, \mathrm{OAc}) \mathrm{ppm} . \mathrm{MS}$ $(70 \mathrm{eV}, \mathrm{EI}): m / z(\%)=451(<1)\left[M-\mathrm{CH}_{3} \mathrm{OH}\right]^{+}, 450(<1), 307$ (19), 247 (26), 88 (100). HRMS (EI): $\mathrm{m} / z$ : calcd. for $\mathrm{C}_{20} \mathrm{H}_{34}{ }^{2} \mathrm{HO}_{11}$ :

$451.2164[M-\mathrm{CH} \mathrm{OH}]^{+}$; found 451.2179 .

Oxidative HAT of Methyl 2,3,4,6-Tetra- $O$-methyl- $\alpha$-D-mannopyranosyl-(1< 4a)-2,3-di- $O$-methyl-4a-carba- $\alpha$-D-glucopyranoside (2): A solution of the alcohol $2(64 \mathrm{mg}, 0.146 \mathrm{mmol})$ in dry $\mathrm{CH}_{2} \mathrm{Cl}_{2}$ $(7 \mathrm{~mL})$ containing DIB $(80 \mathrm{mg}, 0.248 \mathrm{mmol})$ and iodine $(29 \mathrm{mg}$, $0.146 \mathrm{mmol}$ ) under nitrogen was irradiated with two $80 \mathrm{~W}$ tungsten-filament lamps at room temperature for $1.5 \mathrm{~h}$. The mixture was then poured into $10 \%$ aqueous $\mathrm{Na}_{2} \mathrm{~S}_{2} \mathrm{O}_{3}$ and extracted with $\mathrm{CH}_{2} \mathrm{Cl}_{2}$, dried with $\mathrm{Na}_{2} \mathrm{SO}_{4}$ and concentrated under reduced pressure. Chromatography of the residue (hexanes/EtOAc, 2:8< $0: 1$ ) afforded in order of elution: a mixture of methyl (4aS)-1',6-anhydro-(2',3',4',6'-tetra- $O$-methyl- $\alpha$ - - -manno-hex-1'-ulopyranosyl)(1< 4a)-2,3-di- $O$-methyl-4a-iodo-4a-carba- $\alpha$ - $\square$-glucopyranoside (20) and methyl $(2 S, 4 \mathrm{a} S)$-2', 6-anhydro-(2', $3^{\prime}, 4^{\prime}, 6^{\prime}$-tetra- $O$-methyl$\alpha$ - $\square$-arabino-hex-2'-ulopyranosyl)-(1< 4a)-2,3-di- $O$-methyl-4aiodo-4a-carba- $\alpha$ - - -glucopyranoside (21) $(9 \mathrm{mg}, 0.016 \mathrm{mmol}, 9 \%$ ), methyl 1'-6-anhydro-(2',3',4',6'-tetra- $O$-methyl- $\alpha$ - $\square$-manno-hex-1'ulopyranosyl)-( $1<4$ a)-2,3-di- $O$-methyl-4a-carba- $\alpha-\square$-glucopyranoside (19) $(26.1 \mathrm{mg}, 0.06 \mathrm{mmol}, 41 \%)$, and methyl (5'R)-5'-6-anhydro-(2',3',4',6'-tetra- $O$-methyl- $\alpha$ - - -lyxo-hexos-5'-ulopyranosyl)(1< 4a)-2,3-di- $O$-methyl-4a-carba- $\alpha$ - $\square$-glucopyranoside (18) (20 mg, $0.046 \mathrm{mmol}, 32 \%$ ). Careful purification by chromatotron chromatography of the mixture of iodo compounds $\mathbf{2 0}$ and $\mathbf{2 1}$ allowed isolation of small amounts of pure $\mathbf{2 0}$ together with enriched fractions of 21 .

Compound 18: $[\alpha]_{\mathrm{D}}=+38.7\left(c=0.390\right.$, in $\left.\mathrm{CHCl}_{3}\right) .{ }^{1} \mathrm{H} \mathrm{NMR}$ $\left(500 \mathrm{MHz}, \mathrm{C}_{6} \mathrm{D}_{6}\right): \delta=1.70(\mathrm{ddd}, J=7.3,13.6,13.6 \mathrm{~Hz}, 1 \mathrm{H}, 4 \mathrm{a}-$ $\left.\mathrm{H}_{\mathrm{a}}\right), 1.72\left(\mathrm{ddd}, J=1.9,7.3,13.6 \mathrm{~Hz}, 1 \mathrm{H}, 4 \mathrm{a}-\mathrm{H}_{\mathrm{b}}\right), 1.97$ (dddd, $J=$ 1.9, 6.6, 10.4, $10.4 \mathrm{~Hz}, 1 \mathrm{H}, \quad 4-\mathrm{H}), 3.13$ (s, $3 \mathrm{H}, \mathrm{OMe}), 3.15$ (s, $3 \mathrm{H}$, OMe), 3.17 (s, $3 \mathrm{H}, \mathrm{OMe}$ ), 3.21 (s, $3 \mathrm{H}, \mathrm{OMe}$ ), 3.25 (dd, $J=3.5$, $9.5 \mathrm{~Hz}, 1 \mathrm{H}, 2-\mathrm{H}), 3.31\left(\mathrm{dd}, J=1.6,3.0 \mathrm{~Hz}, 1 \mathrm{H}, 2^{\prime}-\mathrm{H}\right), 3.37$ (dd, $J=9.5,10.7 \mathrm{~Hz}, 1 \mathrm{H}, 3-\mathrm{H}), 3.38(\mathrm{~s}, 3 \mathrm{H}, \mathrm{OMe}), 3.45(\mathrm{~d}, J=$ $10.4 \mathrm{~Hz}, 1 \mathrm{H}, 6$ ' $-\mathrm{H}_{\mathrm{a}}$ ), 3.57 (s, $3 \mathrm{H}, \mathrm{OMe}$ ), 3.65 (ddd, $J=3.8,10.4$, $10.4 \mathrm{~Hz}, 1 \mathrm{H}, 5-\mathrm{H}), 3.67$ (s, $3 \mathrm{H}, \mathrm{OMe}), 3.72(\mathrm{~d}, J=10.4 \mathrm{~Hz}, 1 \mathrm{H}$, $\left.6^{\prime}-\mathrm{H}_{\mathrm{b}}\right), 3.89\left(\mathrm{dd}, J=4.1,11.3 \mathrm{~Hz}, 1 \mathrm{H}, 6-\mathrm{H}_{\mathrm{b}}\right), 3.94(\mathrm{dd}, J=2.8$, $\left.10.1 \mathrm{~Hz}, 1 \mathrm{H}, 3^{\prime}-\mathrm{H}\right), 4.05$ (dd, $J=10.7,10.7 \mathrm{~Hz}, 1 \mathrm{H}, 6-\mathrm{H}_{\mathrm{a}}$ ), 4.22 (ddd, $\left.J=1.6,7.3,12.0 \mathrm{~Hz}, 1 \mathrm{H}, 1^{\prime}-\mathrm{H}\right), 4.36(\mathrm{~d}, J=10.4 \mathrm{~Hz}, 1 \mathrm{H}$, $\left.4^{\prime}-\mathrm{H}\right), 4.73$ (d, $\left.J=3.2 \mathrm{~Hz}, 1 \mathrm{H}, 1-\mathrm{H}\right) \mathrm{ppm} .{ }^{13} \mathrm{C} \mathrm{NMR}(125.7 \mathrm{MHz}$, $\left.\mathrm{C}_{6} \mathrm{D}_{6}\right): \delta=31.9\left(\mathrm{CH}_{2}, \mathrm{C}-4 \mathrm{a}\right), 43.9(\mathrm{CH}, \mathrm{C}-4), 54.8\left(\mathrm{CH}_{3}, \mathrm{OMe}\right)$, $57.2\left(\mathrm{CH}_{3}, \mathrm{OMe}\right), 57.5\left(\mathrm{CH}_{3}, \mathrm{OMe}\right), 58.1\left(\mathrm{CH}_{3}, \mathrm{OMe}\right), 59.1\left(\mathrm{CH}_{3}\right.$, $\mathrm{OMe}), 61.0\left(\mathrm{CH}_{3}, \mathrm{OMe}\right), 61.1\left(\mathrm{CH}_{3}, \mathrm{OMe}\right), 65.5\left(\mathrm{CH}_{2}, \mathrm{C}-6\right), 70.7$ (CH, C-5), $73.2\left(\mathrm{CH}_{2}, \mathrm{C}-6\right.$ '), $74.4\left(\mathrm{CH}, \mathrm{C}-1^{\prime}\right), 77.8(\mathrm{CH}, \mathrm{C}-4$ '), 78.7 $\left(\mathrm{CH}, \mathrm{C}-3{ }^{\prime}\right), 79.2(\mathrm{CH}, \mathrm{C}-2 '), 80.3(\mathrm{CH}, \mathrm{C}-3), 84.4(\mathrm{CH}, \mathrm{C}-2), 98.0$ (CH, C-1), 103.4 (C, C-5') ppm. IR (film): $\tilde{v}=2944,2836,1191$, $1141 \mathrm{~cm}^{-1}$. MS $\left(\mathrm{ESI}^{+}\right): \mathrm{m} / z(\%)=459(100)[\mathrm{M}+\mathrm{Na}]^{+}$. HRMS $\left(\mathrm{ESI}^{+}\right)$: $\mathrm{m} / z$ : calcd. for $\mathrm{C}_{20} \mathrm{H}_{36} \mathrm{O}_{10}: 459.2206[\mathrm{M}+\mathrm{Na}]^{+}$; found
459.2203. $\mathrm{C}_{20} \mathrm{H}_{36} \mathrm{O}_{10}$ (436.50): calcd. C 55.03, $\mathrm{H}$ 8.31; found $\mathrm{C}$ $55.33, \mathrm{H} 7.99$.

Compound 19: $[\alpha]_{\mathrm{D}}=+174.8\left(c=0.076\right.$, in $\left.\mathrm{CHCl}_{3}\right) .{ }^{1} \mathrm{H}$ NMR $\quad(500$ $\left.\mathrm{MHz}, \mathrm{C}_{6} \mathrm{D}_{6}\right): \delta=1.11\left(\mathrm{dd}, J=12.3,12.3 \mathrm{~Hz}, 1 \mathrm{H}, 4 \mathrm{a}-\mathrm{H}_{\mathrm{a}}\right)$, 2.25 (dddd, $J=3.7,10.5,10.5,12.1 \mathrm{~Hz}, 1 \mathrm{H}, 4-\mathrm{H}), 2.78(\mathrm{dd}, J=$ $3.7,13.3 \mathrm{~Hz}, 1 \mathrm{H}, 4 \mathrm{a}-\mathrm{H}_{\mathrm{b}}$ ), $3.14(\mathrm{dd}, J=3.4,9.2 \mathrm{~Hz}, 1 \mathrm{H}, 2-\mathrm{H}), 3.17$ (s, $6 \mathrm{H}, 2 \times \mathrm{OMe}), 3.22(\mathrm{~s}, 3 \mathrm{H}, \mathrm{OMe}), 3.33(\mathrm{~s}, 3 \mathrm{H}, \mathrm{OMe}), 3.33(\mathrm{~d}$, $\left.J=3.1 \mathrm{~Hz}, 1 \mathrm{H}, 2^{\prime}-\mathrm{H}\right), 3.37$ (dd, $\left.J=9.3,10.7 \mathrm{~Hz}, 1 \mathrm{H}, 3-\mathrm{H}\right), 3.40$ (s, $3 \mathrm{H}, \mathrm{OMe}$ ), 3.42 (s, $3 \mathrm{H}, \mathrm{OMe}$ ), 3.49 (s, $3 \mathrm{H}, \mathrm{OMe}$ ), 3.59 (dd, $J$ $\left.=1.8,10.7 \mathrm{~Hz}, 1 \mathrm{H}, 6-\mathrm{H}_{\mathrm{a}}\right), 3.65\left(\mathrm{dd}, J=5.4,10.5 \mathrm{~Hz}, 1 \mathrm{H}, 6-\mathrm{H}_{\mathrm{b}}\right)$, $3.70(\mathrm{~m}, 1 \mathrm{H}, 5-\mathrm{H}), 3.75-3.82\left(\mathrm{~m}, 4 \mathrm{H}, 6^{\prime}-\mathrm{H}_{2}, 5^{\prime}-\mathrm{H}, 4^{\prime}-\mathrm{H}\right), 3.88$ $\left(\mathrm{dd}, J=2.9,9.0 \mathrm{~Hz}, 1 \mathrm{H}, 3^{\prime}-\mathrm{H}\right), 4.72(\mathrm{~d}, J=3.5 \mathrm{~Hz}, 1 \mathrm{H}, 1-\mathrm{H})$ ppm. ${ }^{13} \mathrm{C}$ NMR $\left(125.7 \mathrm{MHz}, \mathrm{C}_{6} \mathrm{D}_{6}\right): \delta=33.7\left(\mathrm{CH}_{2}, \mathrm{C}-4 \mathrm{a}\right), 40.7$ (CH, C-4), $54.9\left(\mathrm{CH}_{3}, \mathrm{OMe}\right), 57.7\left(\mathrm{CH}_{3}, \mathrm{OMe}\right), 57.8\left(\mathrm{CH}_{3}, \mathrm{OMe}\right)$, $59.2\left(\mathrm{CH}_{3}, \mathrm{OMe}\right), 60.2\left(\mathrm{CH}_{3}, \mathrm{OMe}\right), 60.5\left(\mathrm{CH}_{3}, \mathrm{OMe}\right), 61.3\left(\mathrm{CH}_{3}\right.$, OMe), $62.6\left(\mathrm{CH}{ }_{2} \mathrm{C}^{-6}\right), 67.1(\mathrm{CH}, \mathrm{C}-5), 72.4(\mathrm{CH}, \mathrm{C}-6), 73.1$ $\left(\mathrm{CH}, \mathrm{C}-5^{\prime}\right), 77.2\left(\mathrm{CH}, \mathrm{C}-4{ }^{\prime}\right), 80.6(\mathrm{CH}, \mathrm{C}-3), 81.2(\mathrm{CH}, \mathrm{C}-2 '), 83.9$ (CH, C-3'), $84.0(\mathrm{CH}, \mathrm{C}-2), 99.1(\mathrm{CH}, \mathrm{C}-1), 99.2\left(\mathrm{C}, \mathrm{C}-1^{\prime}\right) \mathrm{ppm}$. IR (film): $\tilde{v}=2916,2828,1195,1096,960 \mathrm{~cm}^{-1}$. MS (70 eV, EI): $m / z(\%)=436(<1)[\mathrm{M}]^{+}, 391(<1), 261$ (25), 88 (100). HRMS (EI): $\mathrm{m} / z$ : calcd. for $\mathrm{C}_{20} \mathrm{H}_{36} \mathrm{O}_{10}: 436.2308[\mathrm{M}]^{+}$; found 436.2323 . $\mathrm{C}_{20} \mathrm{H}_{36} \mathrm{O}_{10}$ (436.50): calcd. C 55.03, H 8.31; found C 55.21, H 8.13.

Compound 20: ${ }^{1} \mathrm{H}$ NMR $\left(500 \mathrm{MHz}, \mathrm{C}_{6} \mathrm{D}_{6}\right): \delta=1.87$ (ddd, $J=2.8$, $10.1,10.1 \mathrm{~Hz}, 1 \mathrm{H}, 4-\mathrm{H}), 3.09$ (s, $3 \mathrm{H}, \mathrm{OMe}), 3.10$ (s, $3 \mathrm{H}, \mathrm{OMe}$ ), $3.17(\mathrm{~s}, 3 \mathrm{H}, \mathrm{OMe}), 3.23(\mathrm{~s}, 3 \mathrm{H}, \mathrm{OMe}), 3.25(\mathrm{dd}, J=3.5,9.1 \mathrm{~Hz}$, $1 \mathrm{H}, 2-\mathrm{H}), 3.45$ (s, $3 \mathrm{H}, \mathrm{OMe}), 3.53\left(\mathrm{dd}, J=1.6,10.7 \mathrm{~Hz}, 1 \mathrm{H}, 6^{\prime}-\right.$ $\left.\mathrm{H}_{\mathrm{a}}\right), 3.59\left(\mathrm{dd}, J=5.7,10.7 \mathrm{~Hz}, 1 \mathrm{H}, 6^{\prime}-\mathrm{H}_{\mathrm{b}}\right), 3.64$ (s, $\left.3 \mathrm{H}, \mathrm{OMe}\right)$, 3.72 (dd, $\left.J=9.8,9.8 \mathrm{~Hz}, 1 \mathrm{H}, 4^{\prime}-\mathrm{H}\right), 3.76$ (s, $\left.3 \mathrm{H}, \mathrm{OMe}\right), 3.77$ (ddd, $\left.J=1.3,5.7,9.8 \mathrm{~Hz}, 1 \mathrm{H}, 5^{\prime}-\mathrm{H}\right), 3.80-3.87$ (m, $\left.3 \mathrm{H}, 6-\mathrm{H}_{2}, 3^{\prime}-\mathrm{H}\right)$, $3.98(\mathrm{dd}, J=9.8,9.8 \mathrm{~Hz}, 1 \mathrm{H}, 3-\mathrm{H}), 4.27(\mathrm{ddd}, J=6.3,9.8,9.8 \mathrm{~Hz}$, $1 \mathrm{H}, 5-\mathrm{H}), 4.30\left(\mathrm{~d}, J=2.8 \mathrm{~Hz}, 1 \mathrm{H}, 2^{\prime}-\mathrm{H}\right), 4.64(\mathrm{~d}, J=3.5 \mathrm{~Hz}, 1$ $\mathrm{H}, 1-\mathrm{H}), 5.26(\mathrm{~d}, J=2.8 \mathrm{~Hz}, 1 \mathrm{H}, 4 \mathrm{a}-\mathrm{H}) \mathrm{ppm} .{ }^{13} \mathrm{C}$ NMR $\left(125.7 \mathrm{MHz}, \mathrm{C}_{6} \mathrm{D}_{6}\right): \delta=36.7(\mathrm{CH}, \mathrm{C}-4 \mathrm{a}), 44.8(\mathrm{CH}, \mathrm{C}-4), 55.2$ $\left(\mathrm{CH}_{3}, \mathrm{OMe}\right), 57.6\left(\mathrm{CH}_{3}, \mathrm{OMe}\right), 57.8\left(\mathrm{CH}_{3}, \mathrm{OMe}\right), 59.1\left(\mathrm{CH}_{3}\right.$, $\mathrm{OMe}), 60.5\left(\mathrm{CH}_{3}, \mathrm{OMe}\right), 60.9\left(\mathrm{CH}_{3}, \mathrm{OMe}\right), 61.5\left(\mathrm{CH}_{3}, \mathrm{OMe}\right), 63.2$ $\left(\mathrm{CH}_{2}, \mathrm{C}-6\right), 64.3(\mathrm{CH}, \mathrm{C}-5), 72.2\left(\mathrm{CH}_{2}, \mathrm{C}-6{ }^{\prime}\right), 73.8\left(\mathrm{CH}, \mathrm{C}-5^{\prime}\right), 76.9$ $\left(\mathrm{CH}, \mathrm{C}-4^{\prime}\right), 80.1(\mathrm{CH}, \mathrm{C}-3), 80.3\left(\mathrm{CH}, \mathrm{C}-2^{\prime}\right), 84.4\left(\mathrm{CH}, \mathrm{C}-3^{\prime}\right), 85.2$ $(\mathrm{CH}, \mathrm{C}-2), 99.0(\mathrm{CH}, \mathrm{C}-1), 99.9\left(\mathrm{C}, \mathrm{C}-1^{\prime}\right)$ ppm. MS $\left(\mathrm{ESI}^{+}\right)$: $\mathrm{m} / \mathrm{z}(\%)=585(100)[\mathrm{M}+\mathrm{Na}]^{+}$. HRMS $\left(\mathrm{ESI}^{+}\right): \mathrm{m} / \mathrm{z}$ : calcd. for $\mathrm{C}_{20} \mathrm{H}_{35} \mathrm{INaO}_{10}: 585.1186[\mathrm{M}+\mathrm{Na}]^{+}$; found 585.1188 .

Compound 21: From spectroscopic data obtained from an enriched mixture of $\mathbf{2 0}$ and 21, only clearly distinguished signals are reported: ${ }^{1} \mathrm{H}$ NMR $\left(500 \mathrm{MHz}, \mathrm{C}_{6} \mathrm{D}_{6}\right): \delta=1.76$ (ddd, $J=4.1,10.1$, $10.1 \mathrm{~Hz}, 1 \mathrm{H}, 4-\mathrm{H}), 3.10$ (s, $3 \mathrm{H}, \mathrm{OMe}), 3.12$ (s, $3 \mathrm{H}, \mathrm{OMe}), 3.25$ (s, $3 \mathrm{H}, \mathrm{OMe}), 3.38(\mathrm{dd}, J=3.2,8.8 \mathrm{~Hz}, 1 \mathrm{H}, 2-\mathrm{H}), 3.45$ (s, $3 \mathrm{H}$, $\mathrm{OMe}$ ), 3.50 (s, $3 \mathrm{H}, \mathrm{OMe}), 3.52$ (s, $3 \mathrm{H}, \mathrm{OMe}), 3.68$ (dd, $J=8.8$, $8.8 \mathrm{~Hz}, 1 \mathrm{H}), 3.73(\mathrm{~s}, 3 \mathrm{H}, \mathrm{OMe}), 3.98(\mathrm{~m}, 1 \mathrm{H}, 5-\mathrm{H}), 4.15$ (dd, $J$ $=9.5,9.5 \mathrm{~Hz}, 1 \mathrm{H}, 3-\mathrm{H}), 4.59\left(\mathrm{~d}, J=9.5 \mathrm{~Hz}, 1 \mathrm{H}, 1^{\prime}-\mathrm{H}\right), 4.65(\mathrm{~d}$, $J=4.1 \mathrm{~Hz}, 1 \mathrm{H}, 1-\mathrm{H}), 5.29(\mathrm{dd}, J=4.4,9.5 \mathrm{~Hz}, 1 \mathrm{H}, 4 \mathrm{a}-\mathrm{H}) \mathrm{ppm}$. ${ }^{13} \mathrm{C}$ NMR $\left(125.7 \mathrm{MHz}, \mathrm{C}_{6} \mathrm{D}_{6}\right): \delta=31.6(\mathrm{CH}, \mathrm{C}-4 \mathrm{a}), 44.2(\mathrm{CH}, \mathrm{C}-$ 4), $51.4\left(\mathrm{CH}_{3}, \mathrm{OMe}\right), 55.1\left(\mathrm{CH}_{3}, \mathrm{OMe}\right), 57.4\left(\mathrm{CH}_{3}, \mathrm{OMe}\right), 59.3$ $\left(\mathrm{CH}_{3}, \mathrm{OMe}\right), 60.5\left(\mathrm{CH}_{3}, \mathrm{OMe}\right), 61.1\left(\mathrm{CH}_{3}, \mathrm{OMe}\right), 61.5\left(\mathrm{CH}_{3}\right.$, $\mathrm{OMe}), 64.1\left(\mathrm{CH}_{2}, \mathrm{C}-6\right), 69.5(\mathrm{CH}, \mathrm{C}-5), 72.0\left(\mathrm{CH}_{2}, \mathrm{C}-6 \mathrm{\prime}\right), 75.0$ $(\mathrm{CH}), 78.4(\mathrm{CH}), 82.2(\mathrm{CH}, \mathrm{C}-3), 84.3(\mathrm{CH}, \mathrm{C}-2), 84.9\left(\mathrm{CH}, \mathrm{C}-1^{\prime}\right)$, $87.3(\mathrm{CH}), 98.7(\mathrm{CH}, \mathrm{C}-1), 101.1(\mathrm{C}, \mathrm{C}-2$ ') $\mathrm{ppm}$.

Reductive HAT of Methyl 2,3,4,6-Tetra- $O$-methyl- $\alpha$-D-mannopyranosyl-(1<4a)-2,3-di- $O$-methyl-6- $O$-phthalimido-4a-carba- $\alpha$-Dglucopyranoside (22). Method A $\left(n \mathrm{Bu}_{3} \mathrm{SnH} / \mathrm{AIBN}\right)$ : A solution of the phthalimide $22(29 \mathrm{mg}, 0.05 \mathrm{mmol})$ in dry benzene $(3.57 \mathrm{~mL})$ containing $n \mathrm{Bu}_{3} \mathrm{SnH}(13.4 \mu \mathrm{L}, 0.05 \mathrm{mmol})$ and AIBN $(0.8 \mathrm{mg}$, $0.005 \mathrm{mmol}$ ) was heated at reflux for $1 \mathrm{~h}$ under $\mathrm{N}_{2}$. After this time another portion of $n \mathrm{Bu}_{3} \mathrm{SnH}(13.4 \mu \mathrm{L}, 0.05 \mathrm{mmol})$ and $\mathrm{AIBN}$ 
$(0.8 \mathrm{mg}, 0.005 \mathrm{mmol})$ were added and the heating at reflux was continued for an additional $1 \mathrm{~h}$. After cooling to room temperature the mixture was concentrated under reduced pressure. The residue was dissolved in $\mathrm{CH}_{3} \mathrm{CN}$, washed with $n$-hexane and the combined more polar extracts were concentrated under reduced pressure. The residue was purified by chromatotron chromatography $\left(\mathrm{CHCl}_{3} /\right.$ $\mathrm{MeOH}, 99.5: 0.5)$ to give in order of elution methyl (2,3,4,6-tetra$O$-methyl- $\beta$ - $\square$-mannopyranosyl)-( $1<4$ a)-2,3-di- $O$-methyl-4acarba- $\alpha-\square$-glucopyranoside (24) (7 mg, $0.016 \mathrm{mmol}, 32 \%)$ and methyl (2,3,4,6-tetra- $O$-methyl- $\beta$ - $\square$-gulopyranosyl)-( $1<4 \mathrm{a})$-2,3-di$O$-methyl-4a-carba- $\alpha-\square$-glucopyranoside (23) $(3.2 \mathrm{mg}, 0.007 \mathrm{mmol}$, $15 \%)$, and starting alcohol (2) (8.8 mg, $0.02 \mathrm{mmol}, 40 \%)$.

Compound 23: $[\alpha]_{\mathrm{D}}=+109.4\left(c=0.160\right.$, in $\left.\mathrm{CHCl}_{3}\right) .{ }^{1} \mathrm{H}$ NMR $\quad(500$ $\left.\mathrm{MHz}, \mathrm{C}_{6} \mathrm{D}_{6}\right): \delta=1.47$ (ddd, $J=2.8,10.7,14.8 \mathrm{~Hz}, 1 \mathrm{H}, 4 \mathrm{a}-$

$\left.\mathrm{H}_{\mathrm{a}}\right), 2.09\left(\mathrm{ddd}, J=2.2,5.0,14.8 \mathrm{~Hz}, 1 \mathrm{H}, 4 \mathrm{a}-\mathrm{H}_{\mathrm{b}}\right), 2.21(\mathrm{dddd}, J=$ 2.5, 5.1, 10.7, $10.7 \mathrm{~Hz}, 1 \mathrm{H}, 4-\mathrm{H}$ ), 3.107 (s, $3 \mathrm{H}, \mathrm{OMe}$ ), 3.109 (s, 3 $\mathrm{H}, \mathrm{OMe}$ ), 3.13 (s, $3 \mathrm{H}, \mathrm{OMe}$ ), 3.16 (s, $3 \mathrm{H}, \mathrm{OMe}$ ), 3.18 (s, $3 \mathrm{H}$, OMe), 3.20 (m, $2 \mathrm{H}), 3.23$ (s, $3 \mathrm{H}, \mathrm{OMe}), 3.24(\mathrm{dd}, J=1.6,3.8 \mathrm{~Hz}$, $\left.1 \mathrm{H}, 4^{\prime}-\mathrm{H}\right), 3.44\left(\mathrm{dd}, J=6.0,9.1 \mathrm{~Hz}, 1 \mathrm{H}, 6^{\prime}-\mathrm{H}_{\mathrm{a}}\right), 3.58(\mathrm{~m}, 3 \mathrm{H})$, $3.63(\mathrm{~s}, 3 \mathrm{H}, \mathrm{OMe}), 3.65(\mathrm{dd}, J=9.5,9.5 \mathrm{~Hz}, 1 \mathrm{H}, 3-\mathrm{H}), 3.96(\mathrm{~m}$, $\left.1 \mathrm{H}, 6-\mathrm{H}_{\mathrm{a}}\right), 4.01\left(\mathrm{~m}, 1 \mathrm{H}, 6-\mathrm{H}_{\mathrm{b}}\right), 4.08$ (ddd, $J=1.6,7.3,7.3 \mathrm{~Hz}, 1$ $\left.\mathrm{H}, 5^{\prime}-\mathrm{H}\right), 4.15$ (ddd, $\left.J=2.2,10.7,10.7 \mathrm{~Hz}, 1 \mathrm{H}, 1^{\prime}-\mathrm{H}\right), 4.76$ (d, $J$ $=3.5 \mathrm{~Hz}, 1 \mathrm{H}, 1-\mathrm{H}) \mathrm{ppm} .{ }^{13} \mathrm{C} \mathrm{NMR}\left(125.7 \mathrm{MHz}, \mathrm{C}_{6} \mathrm{D}_{6}\right): \delta=31.7$ $\left(\mathrm{CH}_{2}, \mathrm{C}-4 \mathrm{a}\right), 39.6(\mathrm{CH}, \mathrm{C}-4), 54.7\left(\mathrm{CH}_{3}, \mathrm{OMe}\right), 56.7\left(\mathrm{CH}_{3}, \mathrm{OMe}\right)$, $57.5\left(\mathrm{CH}_{3}, \mathrm{OMe}\right), 58.4\left(\mathrm{CH}_{3}, \mathrm{OMe}\right), 58.7\left(\mathrm{CH}_{3}, \mathrm{OMe}\right), 58.9\left(\mathrm{CH}_{3}\right.$, OMe $), 61.0\left(\mathrm{CH}_{3}, \mathrm{OMe}\right), 63.1\left(\mathrm{CH}_{2}, \mathrm{C}-6\right), 71.63\left(\mathrm{CH}_{2}, \mathrm{C}-6{ }^{\prime}\right), 72.9$ $(\mathrm{CH}), 73.3(\mathrm{CH}), 73.54\left(\mathrm{CH}, \mathrm{C}-5^{\prime}\right), 75.5\left(\mathrm{CH}, \mathrm{C}-1^{\prime}\right), 77.75(\mathrm{CH}$, C-4'), $80.7(\mathrm{CH}), 83.2(\mathrm{CH}, \mathrm{C}-3), 84.2(\mathrm{CH}), 98.5(\mathrm{CH}, \mathrm{C}-1) \mathrm{ppm}$. IR (film): $\tilde{v}=3509,2916,1097 \mathrm{~cm}^{-1}$. MS $\left(\mathrm{ESI}^{+}\right): \mathrm{m} / \mathrm{z}(\%)=461$ (100) $[\mathrm{M}+\mathrm{Na}]^{+}$. HRMS $\left(\mathrm{ESI}^{+}\right): \mathrm{m} / \mathrm{z}$ : calcd. for $\mathrm{C}_{20} \mathrm{H}_{38} \mathrm{NaO}_{10}$ : 461.2363 [M + Na $]^{+}$; found 461.2362. $\mathrm{C}_{20} \mathrm{H}_{38} \mathrm{O}_{10}$ (438.52): calcd. $\mathrm{C}$ 54.78, H 8.73; found C 54.83, H 8.37.

Compound 24: $[\alpha]_{\mathrm{D}}=+50.8\left(c=0.240\right.$, in $\left.\mathrm{CHCl}_{3}\right) .{ }^{1} \mathrm{H}$ NMR $(400$ $\left.\mathrm{MHz}, \mathrm{C}_{6} \mathrm{D}_{6}\right): \delta=1.91\left(\mathrm{~m}, 2 \mathrm{H}, 4 \mathrm{a}-\mathrm{H}_{\mathrm{a}}, 4-\mathrm{H}\right), 2.08(\mathrm{~m}, 1 \mathrm{H}, 4 \mathrm{a}-$

$\mathrm{H}_{\mathrm{b}}$ ), $3.02\left(\mathrm{dd}, J=2.8,9.1 \mathrm{~Hz}, 1 \mathrm{H}, 3^{\prime}-\mathrm{H}\right), 3.20$ (s, $\left.3 \mathrm{H}, \mathrm{OMe}\right), 3.21$ (s, $3 \mathrm{H}, \mathrm{OMe}), 3.23(\mathrm{~s}, 3 \mathrm{H}, \mathrm{OMe}), 3.25(\mathrm{dd}, J=3.5,9.1 \mathrm{~Hz}, 1 \mathrm{H}$, 2-H), 3.27 (s, $3 \mathrm{H}, \mathrm{OMe}$ ), 3.29 (dd, $J=1.0,2.8 \mathrm{~Hz}, 1 \mathrm{H}, 2$ '-H), 3.38 (ddd, $\left.J=2.5,6.6,9.1 \mathrm{~Hz}, 1 \mathrm{H}, 5^{\prime}-\mathrm{H}\right), 3.41$ (s, $\left.3 \mathrm{H}, \mathrm{OMe}\right), 3.43$ (ddd, $\left.J=1.0,3.5,9.1 \mathrm{~Hz}, 1 \mathrm{H}, 1^{\prime}-\mathrm{H}\right), 3.47$ (s, $3 \mathrm{H}, \mathrm{OMe}$ ), 3.49 (dd, $\left.J=9.5,9.5 \mathrm{~Hz}, 1 \mathrm{H}, 4^{\prime}-\mathrm{H}\right), 3.51(\mathrm{~s}, 3 \mathrm{H}, \mathrm{OMe}), 3.53(\mathrm{dd}, J=6.6$, $\left.10.4 \mathrm{~Hz}, 1 \mathrm{H}, 6^{\prime}-\mathrm{H}_{\mathrm{a}}\right), 3.56\left(\mathrm{dd}, J=2.2,10.4 \mathrm{~Hz}, 1 \mathrm{H}, 6^{\prime}-\mathrm{H}_{\mathrm{b}}\right), 3.66$ $(\mathrm{dd}, J=9.1,10.1 \mathrm{~Hz}, 1 \mathrm{H}, 3-\mathrm{H}), 3.78(\mathrm{dd}, J=4.7,11.4 \mathrm{~Hz}, 1 \mathrm{H}$,

6- $\left.\mathrm{H}_{\mathrm{a}}\right), 3.91(\mathrm{ddd}, J=2.2,5.4,10.7 \mathrm{~Hz}, 1 \mathrm{H}, 5-\mathrm{H}), 4.07(\mathrm{dd}, J=$ $\left.1.9,11.7 \mathrm{~Hz}, 1 \mathrm{H}, 6-\mathrm{H}_{\mathrm{b}}\right), 4.78(\mathrm{~d}, J=3.5 \mathrm{~Hz}, 1 \mathrm{H}, 1-\mathrm{H}) \mathrm{ppm} . \mathrm{C}^{13}$ NMR (125.7 MHz, $\mathrm{C}_{6} \mathrm{D}{ }_{2}: \delta=29.33\left(\mathrm{CH}_{2} \mathrm{C}-4 \mathrm{a}\right), 40.6(\mathrm{CH}, \mathrm{C}-4)$, $54.6\left(\mathrm{CH}_{3}, \mathrm{OMe}\right), 57.3\left(\mathrm{CH}_{3}, \mathrm{OMe}\right), 57.4\left(\mathrm{CH}_{3}, \mathrm{OMe}\right), 58.8\left(\mathrm{CH}_{3}\right.$, $\mathrm{OMe}), 60.0\left(\mathrm{CH}_{3}, \mathrm{OMe}\right), 60.6\left(\mathrm{CH}_{3}, \mathrm{OMe}\right), 61.1\left(\mathrm{CH}_{3}, \mathrm{OMe}\right), 64.0$ $\left(\mathrm{CH}_{2}, \mathrm{C}-6\right), 72.6(\mathrm{CH}, \mathrm{C}-5), 73.0\left(\mathrm{CH}_{2}, \mathrm{C}-6^{\prime}\right), 75.64\left(\mathrm{CH}, \mathrm{C}-1^{\prime}\right)$, $77.6\left(\mathrm{CH}, \mathrm{C}-4^{\prime}\right), 79.00\left(\mathrm{CH}, \mathrm{C}-5^{\prime}\right), 79.02\left(\mathrm{CH}, \mathrm{C}-2^{\prime}\right), 79.7(\mathrm{CH}, \mathrm{C}-$ 3), $84.2(\mathrm{CH}, \mathrm{C}-2), 87.7$ (CH, C-3'), 97.7 ( $\mathrm{CH}, \mathrm{C}-1)$ ppm. IR (film): $\tilde{v}=3483,2925,1094 \mathrm{~cm}^{-1}$. MS $\left(\mathrm{ESI}^{+}\right): \mathrm{m} / z,(\%)=461(100)[\mathrm{M}+$ $\mathrm{Na}]^{+}$. HRMS $\left(\mathrm{ESI}^{+}\right): \mathrm{m} / z$ : calcd. for $\mathrm{C}_{20} \mathrm{H}_{38} \mathrm{NaO}_{10}: 461.2363[\mathrm{M}+$ $\mathrm{Na}]^{+}$; found 461.2365. $\mathrm{C}_{20} \mathrm{H}_{38} \mathrm{O}_{10}$ (438.52): calcd. C 54.78, $\mathrm{H} 8.73$; found C 54.68, H 8.64.

Method B ( $n$ Bu SnD/AIBN): A solution of the phthalimide 22 (30 mg, $0.051 \mathrm{mmol})$ in dry benzene $\left(3.8 \mathrm{~mL}\right.$ ) containing $n \mathrm{Bu}_{3} \mathrm{SnD}$ $(13.8 \mu \mathrm{L}, 0.051 \mathrm{mmol})$ and AIBN $(0.8 \mathrm{mg}, 0.005 \mathrm{mmol})$ was heated at reflux for $2 \mathrm{~h}$ under $\mathrm{N}_{2}$. After this time another portion of $n \mathrm{Bu}_{3} \mathrm{SnD}(13.8 \mu \mathrm{L}, 0.051 \mathrm{mmol})$ and AIBN $(0.8 \mathrm{mg}, 0.005 \mathrm{mmol})$ were added and the heating at reflux was continued for an additional $1 \mathrm{~h}$. After cooling to room temperature the mixture was concentrated under reduced pressure. The residue was dissolved in
$\mathrm{CH}_{3} \mathrm{CN}$, washed with $n$-hexane and the combined more polar extracts were concentrated under reduced pressure. The residue was purified by chromatotron chromatography $\left(\mathrm{CHCl}_{3} / \mathrm{MeOH}\right.$, 99.5:0.5) to afford in order of elution methyl 2,3,4,6-tetra- $O$ methyl- $\beta$ - $\square-\left(1{ }^{\prime}-{ }^{2} \mathrm{H}\right)$ mannopyranosyl-( $\left.1<4 \mathrm{a}\right)-2,3$-di- $O$-methyl-4acarba- $\alpha$ - $\square$-glucopyranoside (26) $(6.4 \mathrm{mg}, 0.014 \mathrm{mmol}, 28 \%$ ), methyl 2,3,4,6-tetra- $O$-methyl- $\beta-\square\left(5^{\prime}-{ }^{2} \mathrm{H}\right)$ gulopyranosyl-(1< 4a)-2,3-di- $O$ methyl-4a-carba- $\alpha$ - $\square$-glucopyranoside (25) $(3.2 \mathrm{mg}, 0.007 \mathrm{mmol}$, $14 \%$ ) and deuterated starting alcohol (27) $(5.6 \mathrm{mg}, 0.013 \mathrm{mmol}$, $\left.25 \%,{ }^{1} \mathrm{H} /{ }^{2} \mathrm{H}-1^{1} /{ }^{2} \mathrm{H}-5^{1} /{ }^{2} \mathrm{H}-4 \mathrm{a}, 54: 11: 11: 24\right)$.

Compound 25: ${ }^{1} \mathrm{H}$ NMR $\left(500 \mathrm{MHz}, \mathrm{C}_{6} \mathrm{D}_{6}\right)$ : (most significant signals) $\delta=1.47$ (ddd, $\left.J=2.8,10.7,14.8 \mathrm{~Hz}, 1 \mathrm{H}, 4 \mathrm{a}-\mathrm{H}_{\mathrm{a}}\right), 2.09$ (ddd, $\left.J=2.2,5.1,14.8 \mathrm{~Hz}, 1 \mathrm{H}, 4 \mathrm{a}-\mathrm{H}_{\mathrm{b}}\right), 2.21$ (dddd, $J=2.5,5.0,10.7$, $10.7 \mathrm{~Hz}, 1 \mathrm{H}, 4-\mathrm{H}), 3.11(\mathrm{~s}, 6 \mathrm{H}, 2 \times \mathrm{OMe}), 3.13(\mathrm{~s}, 3 \mathrm{H}, \mathrm{OMe})$, 3.16 (s, $3 \mathrm{H}, \mathrm{OMe}$ ), 3.18 (s, $3 \mathrm{H}, \mathrm{OMe}), 3.23$ (s, $3 \mathrm{H}, \mathrm{OMe}), 3.63$ (s, $3 \mathrm{H}, \mathrm{OMe}), 3.98\left(\mathrm{~m}, 2 \mathrm{H}, 6-\mathrm{H}_{2}\right), 4.15$ (ddd, $J=2.2,10.7,10.7 \mathrm{~Hz}, 1$ $\left.\mathrm{H}, 11^{\prime}-\mathrm{H}\right), 4.76(\mathrm{~d}, J=3.5 \mathrm{~Hz}, 1 \mathrm{H}, 1-\mathrm{H}) \mathrm{ppm} .{ }^{13} \mathrm{C} \mathrm{NMR}$ $\left(125.7 \mathrm{MHz}, \mathrm{C}_{6} \mathrm{D}_{6}\right): \delta=31.7\left(\mathrm{CH}_{2}, \mathrm{C}-4 \mathrm{a}\right), 39.6(\mathrm{CH}, \mathrm{C}-4), 54.7$ $\left(\mathrm{CH}_{3}, \mathrm{OMe}\right), 56.7\left(\mathrm{CH}_{3}, \mathrm{OMe}\right), 57.5\left(\mathrm{CH}_{3}, \mathrm{OMe}\right), 58.4\left(\mathrm{CH}_{3}\right.$, $\mathrm{OMe}), 58.7\left(\mathrm{CH}_{3}, \mathrm{OMe}\right), 58.9\left(\mathrm{CH}_{3}, \mathrm{OMe}\right), 61.0\left(\mathrm{CH}_{3}, \mathrm{OMe}\right), 63.1$ $\left(\mathrm{CH}_{2}, \mathrm{C}-6\right), 71.55\left(\mathrm{CH}_{2}, \mathrm{C}-6{ }^{\prime}\right), 72.9(\mathrm{CH}), 73.3(\mathrm{CH}), 75.5(\mathrm{CH}, \mathrm{C}-$ $\left.1^{\prime}\right), 77.68\left(\mathrm{CH}, \mathrm{C}-4{ }^{\prime}\right), 80.7(\mathrm{CH}, \mathrm{C}-), 83.2(\mathrm{CH}, \mathrm{C}-3), 84.2(\mathrm{CH})$, $98.5(\mathrm{CH}, \mathrm{C}-1) \mathrm{ppm}$. MS $\left(\mathrm{ESI}^{+}\right): \mathrm{m} / z(\%)=462(100)[\mathrm{M}+\mathrm{Na}]^{+}$. HRMS $\left(\mathrm{ESI}^{+}\right)$: $\mathrm{m} / \mathrm{z}$ : calcd. for $\mathrm{C}_{20} \mathrm{H}_{37}{ }^{2} \mathrm{HNaO}_{10}$ : $462.2425[\mathrm{M}+$ $\mathrm{Na}]^{+}$; found 462.2429 .

Compound 26: ${ }^{1} \mathrm{H}$ NMR $\left(500 \mathrm{MHz}, \mathrm{C}_{6} \mathrm{D}_{6}\right): \delta=1.89(\mathrm{dd}, J=3.5$, $14.5 \mathrm{~Hz}, 1 \mathrm{H}, 4 \mathrm{a}-\mathrm{H}_{\mathrm{a}}$ ), 1.92 (dddd, $J=3.2,4.7,10.7,10.7 \mathrm{~Hz}, 1 \mathrm{H}$, 4-H), 2.08 (br. dd, $J=4.4,14.2 \mathrm{~Hz}, 1 \mathrm{H}, 4 \mathrm{a}-\mathrm{H}_{\mathrm{b}}$ ), 3.02 (dd, $J=2.9$, $\left.9.0 \mathrm{~Hz}, 1 \mathrm{H}, 3^{\prime}-\mathrm{H}\right), 3.19$ (s, $\left.3 \mathrm{H}, \mathrm{OMe}\right), 3.20$ (s, $\left.3 \mathrm{H}, \mathrm{OMe}\right), 3.22$ (s, $3 \mathrm{H}, \mathrm{OMe}), 3.25(\mathrm{dd}, J=3.5,9.2 \mathrm{~Hz}, 1 \mathrm{H}, 2-\mathrm{H}), 3.27(\mathrm{~s}, 3 \mathrm{H}$, OMe), $3.29\left(\mathrm{~d}, J=2.8 \mathrm{~Hz}, 1 \mathrm{H}, 2^{\prime}-\mathrm{H}\right), 3.38$ (ddd, $J=2.2,6.3$, $\left.9.5 \mathrm{~Hz}, 1 \mathrm{H}, 5^{\prime}-\mathrm{H}\right), 3.41$ (s, $\left.3 \mathrm{H}, \mathrm{OMe}\right), 3.47$ (s, $3 \mathrm{H}, \mathrm{OMe}$ ), 3.49 $\left(\mathrm{dd}, J=9.5,9.5 \mathrm{~Hz}, 1 \mathrm{H}, 4^{\prime}-\mathrm{H}\right), 3.51(\mathrm{~s}, 3 \mathrm{H}, \mathrm{OMe}), 3.53(\mathrm{dd}, J=$ $\left.6.3,10.4 \mathrm{~Hz}, 1 \mathrm{H}, 6^{\prime}-\mathrm{H}_{\mathrm{a}}\right), 3.56\left(\mathrm{dd}, J=2.5,10.4 \mathrm{~Hz}, 1 \mathrm{H}, 6^{\prime}-\mathrm{H}_{\mathrm{b}}\right)$, $3.66(\mathrm{dd}, J=9.0,10.3 \mathrm{~Hz}, 1 \mathrm{H}, 3-\mathrm{H}), 3.79\left(\mathrm{~m}, 1 \mathrm{H}, 6-\mathrm{H}_{\mathrm{a}}\right), 3.91$ $(\mathrm{ddd}, J=2.2,5.4,10.7 \mathrm{~Hz}, 1 \mathrm{H}, 5-\mathrm{H}), 4.09(\mathrm{dd}, J=1.9,11.7 \mathrm{~Hz}, 1$ $\left.\mathrm{H}, 6-\mathrm{H}_{\mathrm{b}}\right), 4.79(\mathrm{~d}, J=3.5 \mathrm{~Hz}, 1 \mathrm{H}, 1-\mathrm{H}) \mathrm{ppm} .{ }^{13} \mathrm{C} \mathrm{NMR}$ $\left(100.6 \mathrm{MHz}, \mathrm{C}_{6} \mathrm{D}_{6}\right): \delta=29.25\left(\mathrm{CH}_{2}, \mathrm{C}-4 \mathrm{a}\right), 40.6(\mathrm{CH}, \mathrm{C}-4), 54.6$ $\left(\mathrm{CH}_{3}, \mathrm{OMe}\right), 57.3\left(\mathrm{CH}_{3}, \mathrm{OMe}\right), 57.4\left(\mathrm{CH}_{3}, \mathrm{OMe}\right), 58.8\left(\mathrm{CH}_{3}\right.$, $\mathrm{OMe}), 60.0\left(\mathrm{CH}_{3}, \mathrm{OMe}\right), 60.6\left(\mathrm{CH}_{3}, \mathrm{OMe}\right), 61.1\left(\mathrm{CH}_{3}, \mathrm{OMe}\right), 64.0$ $\left(\mathrm{CH}_{2}, \mathrm{C}-6\right), 72.6(\mathrm{CH}, \mathrm{C}-5), 73.0\left(\mathrm{CH}_{2}, \mathrm{C}-6 \mathrm{\prime}\right), 77.6\left(\mathrm{CH}, \mathrm{C}-4{ }^{\prime}\right)$, 78.88 (CH, C-2'), 78.99 (CH, C-5'), $79.7(\mathrm{CH}, \mathrm{C}-3), 84.1(\mathrm{CH}, \mathrm{C}-$ 2), $87.7\left(\mathrm{CH}, \mathrm{C}-3^{\prime}\right), 97.7(\mathrm{CH}, \mathrm{C}-1) \mathrm{ppm}$. MS (ESI $\left.{ }^{+}\right): \mathrm{m} / \mathrm{z}(\%)=462$ (100) $[\mathrm{M}+\mathrm{Na}]^{+}$. HRMS $\left(\mathrm{ESI}^{+}\right): \mathrm{m} / z$ : calcd. for $\mathrm{C}_{20}{ }^{2} \mathrm{H}_{3} \mathrm{NaO}:{ }_{10}$ $462.2425[\mathrm{M}+\mathrm{Na}]^{+}$; found 462.2431 .

Compound 27: ${ }^{13} \mathrm{C}$ NMR $\left(100.6 \mathrm{MHz}, \mathrm{C}_{6} \mathrm{D}_{6}\right): \delta=30.15\left(\mathrm{CH}_{2}, \mathrm{C}-\right.$ 4a-D), 30.39 ( $\left.\mathrm{CH}_{2}, \mathrm{C}-4 \mathrm{a}\right), 39.89$ (CH, C-4-D), 39.98 (CH, C-4), $54.7\left(\mathrm{CH}_{3}, \mathrm{OMe}\right), 57.0\left(\mathrm{CH}_{3}, \mathrm{OMe}\right), 57.4\left(\mathrm{CH}_{3}, \mathrm{OMe}\right), 57.9\left(\mathrm{CH}_{3}\right.$, $\mathrm{OMe}), 58.4\left(\mathrm{CH}_{3}, \mathrm{OMe}\right), 58.9\left(\mathrm{CH}_{3}, \mathrm{OMe}\right), 60.9\left(\mathrm{CH}_{3}, \mathrm{OMe}\right), 62.9$ $\left(\mathrm{CH}_{2}, \mathrm{C}-6\right), 71.56\left(\mathrm{CH}_{2}, \mathrm{C}-6\right.$ '-D), $71.67\left(\mathrm{CH}_{2}, \mathrm{C}-6\right.$ '), $71.81(\mathrm{CH}$, C-1'-D), 71.87 (CH, C-1'), 72.95 (CH, C-4'-D), 72.99 (CH, C-4'), $73.66\left(\mathrm{CH}, \mathrm{C}-5^{\prime}\right), 77.4$ (CH, C-5), $78.4(\mathrm{CH}, \mathrm{C}-3$ '), 79.1 (CH, C2'), 82.9 (CH, C-3), $84.2(\mathrm{CH}, \mathrm{C}-2), 98.3(\mathrm{CH}, \mathrm{C}-1)$ ppm. MS $\left(\mathrm{ESI}^{+}\right): \mathrm{m} / \mathrm{z}(\%)=462(100)[\mathrm{M}+\mathrm{Na}]^{+}, 461(15)[\mathrm{M}+\mathrm{Na}]^{+}$. HRMS $\left(\mathrm{ESI}^{+}\right): \mathrm{m} / z$ : calcd. for $\mathrm{C}_{20} \mathrm{H}_{37}{ }^{2} \mathrm{HNaO}_{10}: 462.2425[\mathrm{M}+$ $\mathrm{Na}]^{+}$; found 462.2425; calcd. for $\mathrm{C}_{20} \mathrm{H}_{38} \mathrm{NaO}_{10}$ : $461.2363[\mathrm{M}+$ $\mathrm{Na}]^{+}$; found 461.2362 .

Oxidative HAT of 3-Hydroxypropyl 2,3,4,6-Tetra- $O$-methyl- $\alpha$-Dmannopyranoside (28): A solution of the alcohol $28(43 \mathrm{mg}$, $0.146 \mathrm{mmol})$ in dry $\mathrm{CH}_{2} \mathrm{Cl}_{2}(6 \mathrm{~mL})$ containing DIB $(80 \mathrm{mg}$, $0.248 \mathrm{mmol}$ ) and iodine $(37 \mathrm{mg}, 0.146 \mathrm{mmol}$ ) was irradiated with two $80 \mathrm{~W}$ tungsten-filament lamps at room temperature under ni- 
trogen for $5.5 \mathrm{~h}$. After this time another portion of DIB ( $80 \mathrm{mg}$, $0.248 \mathrm{mmol}$ ) and iodine (37 $\mathrm{mg}, 0.146 \mathrm{mmol}$ ) was added and irradiation was continued for an additional $2.5 \mathrm{~h}$. The reaction mixture was then poured into water, extracted with $\mathrm{CH}_{2} \mathrm{Cl}_{2}$ and the organic layer washed with $10 \%$ aqueous $\mathrm{Na}_{2} \mathrm{~S}_{2} \mathrm{O}_{3}$, dried with $\mathrm{Na}_{2} \mathrm{SO}_{4}$ and the solvents evaporated in vacuo. The residue was purified by chromatotron chromatography (hexanes/EtOAc, 35:65) to give in order of elution the known 2,3,4,6-tetra- $O$-methyl- $\square$-mannono-1,5-lactone $(\mathbf{3 0})^{[21]}(4.7 \mathrm{mg}, 0.02 \mathrm{mmol}, 14 \%)$ and 2,3,4,6-tetra- $O$-methyl$\square$-lyxo-hexos-5-ulose propane-1,3-diyl diacetal (29) (16.3 mg, $0.056 \mathrm{mmol}, 38 \%$ ).

Compound 29: M. p. $60.6-62.5^{\circ} \mathrm{C}$ ( $n$-hexane/acetone). $[\alpha]_{\overline{\mathrm{D}}}-40.5$ $\left(c=0.365\right.$, in $\left.\mathrm{CHCl}_{3}\right)$. H NMR $\left(500 \mathrm{MHz}, \mathrm{CDCl}_{3}\right): \delta=1.35$ (ddddd, $J=1.3,1.3,2.6,2.6,13.5 \mathrm{~Hz}, 1 \mathrm{H}, 2-\mathrm{H}_{\mathrm{a}}$ ), 2.17 (ddddd, $J$ $\left.=5.0,5.0,12.5,12.5,12.5 \mathrm{~Hz}, 1 \mathrm{H}, 2-\mathrm{H}_{\mathrm{b}}\right), 3.32(\mathrm{~s}, 3 \mathrm{H}, \mathrm{OMe}), 3.38$ (dd, $\left.J=1.7,8.9 \mathrm{~Hz}, 1 \mathrm{H}, 2^{\prime}-\mathrm{H}\right), 3.43$ (s, $\left.3 \mathrm{H}, \mathrm{OMe}\right), 3.45$ (s, $3 \mathrm{H}$, $\mathrm{OMe}$ ), 3.51 (s, $3 \mathrm{H}, \mathrm{OMe}$ ), $3.68\left(\mathrm{dd}, J=3.2,8.8 \mathrm{~Hz}, 1 \mathrm{H}, 3^{\prime}-\mathrm{H}\right)$, $3.74-3.80(\mathrm{~m}, 2 \mathrm{H}), 3.92\left(\mathrm{~d}, J=3.2 \mathrm{~Hz}, 1 \mathrm{H}, 4^{\prime}-\mathrm{H}\right), 4.15-4.21 \quad(\mathrm{~m}$, $2 \mathrm{H}), 4.33\left(\mathrm{~d}, J=18.2 \mathrm{~Hz}, 1 \mathrm{H}, 6^{\prime}-\mathrm{H}_{\mathrm{a}}\right), 4.39(\mathrm{~d}, J=18.2 \mathrm{~Hz}, 1 \mathrm{H}$, $\left.6^{\prime}-\mathrm{H}_{\mathrm{b}}\right), \quad 4.71\left(\mathrm{~d}, \quad J=1.7 \mathrm{~Hz}, 1 \mathrm{H}, 1^{\prime}-\mathrm{H}\right)$ ppm. ${ }^{13} \mathrm{C} \mathrm{NMR}$ (125.7 MHz, $\left.\mathrm{CDCl}_{3}\right): \delta=25.7\left(\mathrm{CH}_{2}, \mathrm{C}-2\right), 59.4\left(\mathrm{CH}_{3}, \mathrm{OMe}\right), 59.8$ $\left(\mathrm{CH}_{3}, \mathrm{OMe}\right), 60.2\left(\mathrm{CH}_{3}, \mathrm{OMe}\right), 60.5\left(\mathrm{CH}_{3}, \mathrm{OMe}\right), 67.0\left(\mathrm{CH}_{2}\right), 67.4$ $\left(\mathrm{CH}_{2}\right), 76.7\left(\mathrm{CH}_{2}, \mathrm{C}-6^{\prime}\right), 79.4\left(\mathrm{CH}, \mathrm{C}-2^{\prime}\right), 80.9\left(\mathrm{CH}, \mathrm{C}-3^{\prime}\right), 86.5$ (CH, C-4'), $101.1\left(\mathrm{CH}, \mathrm{C}-1^{\prime}\right), 208.3\left(\mathrm{C}, \mathrm{C}-5^{\prime}\right) \mathrm{ppm}$. IR (film): $\tilde{v}=$ 2929, 2849, 1731, $1103 \mathrm{~cm}^{-1}$. MS $(70 \mathrm{eV}, \mathrm{EI}): \mathrm{m} / z(\%)=292(<1)$ $[\mathrm{M}]^{+}, 247$ (1), 219 (8), 131 (15), 87 (100). HRMS (EI): $\mathrm{m} / z$ : calcd. for $\mathrm{C}_{13} \mathrm{H}_{24} \mathrm{O}_{7}$ : $292.1522[\mathrm{M}]^{+}$; found 292.1484. $\mathrm{C}_{13} \mathrm{H}_{24} \mathrm{O}_{7}$ (292.33): calcd. C 53.41, H 8.28; found C 53.60, H 8.35.

Compound 30: $[\alpha]_{\mathrm{D}}=+56.1\left(c=0.130\right.$, in $\left.\mathrm{CHCl}_{3}\right) .{ }^{1} \mathrm{H} \mathrm{NMR} \quad(500$ $\left.\mathrm{MHz}, \mathrm{CDCl}_{3}\right): \delta=3.42(\mathrm{~s}, 3 \mathrm{H}, \mathrm{OMe}), 3.46$ (s, $\left.3 \mathrm{H}, \mathrm{OMe}\right)$, 3.51 (s, $3 \mathrm{H}, \mathrm{OMe}), 3.54$ (dd, $J=1.4,7.4 \mathrm{~Hz}, 1 \mathrm{H}, 4-\mathrm{H}), 3.60$ (s, 3 $\mathrm{H}, \mathrm{OMe}), 3.62\left(\mathrm{dd}, J=3.8,10.9 \mathrm{~Hz}, 1 \mathrm{H}, 6-\mathrm{H}_{\mathrm{a}}\right), 3.65(\mathrm{dd}, J=5.1$, $\left.10.9 \mathrm{~Hz}, 1 \mathrm{H}, 6-\mathrm{H}_{\mathrm{b}}\right), 3.84(\mathrm{dd}, J=1.6,2.8 \mathrm{~Hz}, 1 \mathrm{H}, 3-\mathrm{H}), 4.07(\mathrm{~d}$, $J=2.8 \mathrm{~Hz}, 1 \mathrm{H}, 2-\mathrm{H}), 4.17(\mathrm{ddd}, J=3.7,5.0,7.1 \mathrm{~Hz}, 1 \mathrm{H}, 5-\mathrm{H})$ ppm. ${ }^{13} \mathrm{C}$ NMR $\left(125.7 \mathrm{MHz}, \mathrm{CDCl}_{3}\right): \delta=57.6\left(\mathrm{CH}_{3}, \mathrm{OMe}\right), 58.7$ $\left(\mathrm{CH}_{3}, \mathrm{OMe}\right), 59.2\left(\mathrm{CH}_{3}, \mathrm{OMe}\right), 59.5\left(\mathrm{CH}_{3}, \mathrm{OMe}\right), 71.8\left(\mathrm{CH}_{2}, \mathrm{C}-\right.$ 6), 77.2 (CH, C-4), 77.6 (CH, C-2), $78.2(\mathrm{CH}, \mathrm{C}-5), 78.9(\mathrm{CH}, \mathrm{C}-$ 3), 168.9 (C, C-1) ppm. IR (film): $\tilde{v}=2932,2832,1772,1186,1100$ $\mathrm{cm}^{-1}$. MS $(70 \mathrm{eV}, \mathrm{EI}): \mathrm{m} / z(\%)=234(7)[\mathrm{M}]^{+}, 161(2), 101(85), 88$ (100). HRMS (EI): $m / z$ : calcd. for $\mathrm{C}_{1} \mathrm{H}_{18} \mathrm{O}_{6}: 234.1103[\mathrm{M}]^{+}$; found 234.1094. $\mathrm{C}_{10} \mathrm{H}_{18} \mathrm{O}_{6}$ (234.25): calcd. C 51.27, H 7.75; found C 51.30, H 7.74 .

Reductive HAT of 3-(Phthalimidoxy)propyl 2,3,4,6-Tetra- $O$-methyla-D-mannopyranoside (35). Method A $\left(\boldsymbol{n B u}_{3} \mathrm{SnH} / \mathrm{AIBN}\right)$ : A solution of phthalimide 35 (115 mg, $0.262 \mathrm{mmol})$ in dry benzene $(19.5 \mathrm{~mL}$ ) containing $n \mathrm{Bu}_{3} \mathrm{SnH}(70 \mu \mathrm{L}, 0.264 \mathrm{mmol})$ and AIBN $(4.3 \mathrm{mg}$, $0.026 \mathrm{mmol}$ ) was heated at reflux temperature for $1.5 \mathrm{~h}$. After this time another portion of $n \mathrm{Bu}_{3} \mathrm{SnH}(70 \mu \mathrm{L}, 0.264 \mathrm{mmol})$ and AIBN $(4.3 \mathrm{mg}, 0.026 \mathrm{mmol})$ were added and heating at reflux was continued for an additional $1.5 \mathrm{~h}$. After cooling to room temperature the reaction mixture was concentrated under reduced pressure. The residue was dissolved in $\mathrm{CH}_{3} \mathrm{CN}$, washed with $n$-hexane and the combined more polar extracts were concentrated under reduced pressure. Acetylation of the crude residue in dry pyridine $(3 \mathrm{~mL})$ containing $\mathrm{Ac}_{2} \mathrm{O}(1 \mathrm{~mL})$ at room temperature for $4 \mathrm{~h}$ gave after column chromatography (hexanes-EtOAc, 65:35 < 55:45) the following compounds (in order of elution): 3-acetyloxypropyl 2,3,4,6-tetra$O$-methyl- $\beta$ - $\square$-gulopyranoside (36) (16.6 mg, $0.049 \mathrm{mmol}, 18 \%$ ), 3acetyloxypropyl $2,3,4,6$-tetra- $O$-methyl- $\alpha$ - $\square$-mannopyranoside (37) (26 mg, $0.077 \mathrm{mmol}, 29 \%$ ), and 3-acetyloxypropyl 2,3,4,6-tetra- $O$ methyl- $\beta$ - $\square$-mannopyranoside (38) (11.1 $\mathrm{mg}, 0.033 \mathrm{mmol}, 13 \%$ ), as colourless oils.
Compound 36: $[\alpha]_{\mathrm{D}}=+48.4\left(c=0.320\right.$, in $\left.\mathrm{CHCl}_{3}\right) .{ }^{1} \mathrm{H}$ NMR $\quad(500$ $\left.\mathrm{MHz}, \mathrm{CDCl}_{3}\right): \delta=1.89-1.95\left(\mathrm{~m}, 2 \mathrm{H}, 2-\mathrm{H}_{2}\right), 2.02(\mathrm{~s}, 3 \mathrm{H}$, OMe), 3.25 (dd, $\left.J=3.4,8.4 \mathrm{~Hz}, 1 \mathrm{H}, 2^{\prime}-\mathrm{H}\right), 3.37$ (dd, $J=1.5$, $\left.3.7 \mathrm{~Hz}, 1 \mathrm{H}, 4^{\prime}-\mathrm{H}\right), 3.38$ (s, $\left.3 \mathrm{H}, \mathrm{OMe}\right), 3.44$ (s, $\left.3 \mathrm{H}, \mathrm{OMe}\right), 3.47$ (s, $3 \mathrm{H}, \mathrm{OMe}), 3.51\left(\mathrm{~m}, 1 \mathrm{H}, 6\right.$ 6'- $_{\mathrm{a}}$ ), 3.52 (s, $\left.3 \mathrm{H}, \mathrm{OMe}\right), 3.55-3.60$ $\left(\mathrm{m}, 2 \mathrm{H}, 66^{\prime}-\mathrm{H}_{\mathrm{b}}, 1-\mathrm{H}_{\mathrm{a}}\right), 3.76\left(\mathrm{dd}, J=3.5,3.5 \mathrm{~Hz}, 1 \mathrm{H}, 3^{\prime}-\mathrm{H}\right), 3.93-$ $3.97\left(\mathrm{~m}, 2 \mathrm{H}, 5^{\prime}-\mathrm{H}, 1-\mathrm{H}_{\mathrm{b}}\right), 4.15\left(\mathrm{t}, J=6.5 \mathrm{~Hz}, 2 \mathrm{H}, 3-\mathrm{H}_{2}\right), 4.64(\mathrm{~d}$, $\left.J=8.0 \mathrm{~Hz}, 1 \mathrm{H}, 11^{\prime}-\mathrm{H}\right) \mathrm{ppm} .{ }^{13} \mathrm{C} \mathrm{NMR}\left(125.7 \mathrm{MHz}, \mathrm{CDCl}_{3}\right): \delta=$ $20.9\left(\mathrm{CH}_{3}, \mathrm{OAc}\right), 29.0\left(\mathrm{CH}_{2}, \mathrm{C}-2\right), 58.8\left(\mathrm{CH}_{3}, \mathrm{OMe}\right), 59.1\left(\mathrm{CH}_{3}\right.$, $\mathrm{OMe}), 59.2\left(\mathrm{CH}_{3}, \mathrm{OMe}\right), 59.6\left(\mathrm{CH}_{3}, \mathrm{OMe}\right), 61.7\left(\mathrm{CH}_{2}, \mathrm{C}-3\right), 65.8$ $\left(\mathrm{CH}_{2}, \mathrm{C}-1\right), 71.0\left(\mathrm{CH}_{2}, \mathrm{C}^{-} 6^{\prime}\right), 71.5\left(\mathrm{CH}, \mathrm{C}-5^{\prime}\right), 75.9\left(\mathrm{CH}, \mathrm{C}-4^{\prime}\right)$, 76.7 (CH, C-3'), 78.2 (CH, C-2'), $100.8\left(\mathrm{CH}, \mathrm{C}-1^{\prime}\right), 171.1$ (C, OAc) ppm. IR (film): $\tilde{v}=1736 \mathrm{~cm}^{-1}$. MS $(70 \mathrm{eV}, \mathrm{EI}): \mathrm{m} / z(\%)=277(2)$ $\left[M-\mathrm{C}_{2} \mathrm{H}_{3} \mathrm{O}_{2}\right]^{+}, 219$ (4), 187 (8), 101 (100). HRMS (EI): $m / z$ : calcd. for $\mathrm{C}_{13} \mathrm{H}_{25} \mathrm{O}_{6}$ : 277.1651 $\left[M-\mathrm{C}_{2} \mathrm{H}_{3} \mathrm{O}_{2}\right]^{+}$; found 277.1709. $\mathrm{C}_{15} \mathrm{H}_{28} \mathrm{O}_{8}$ (336.38): calcd. C 53.56, H 8.39; found C 53.66, H 8.12.

Compound 37: $[\alpha]_{\mathrm{D}}=+49.7\left(c=1.190\right.$, in $\left.\mathrm{CHCl}_{3}\right) .{ }^{1} \mathrm{H}$ NMR $\quad(500$ $\mathrm{MHz}, \mathrm{CDCl}_{3}$ ): $\delta=1.88$ (qui, $J=6.3 \mathrm{~Hz}, 2 \mathrm{H}, 2-\mathrm{H}_{2}$ ), 2.02 (s,

$3 \mathrm{H}, \mathrm{OMe}$ ), 3.37 (s, $3 \mathrm{H}, \mathrm{OMe}), 3.38$ (dd, $J=9.4,9.4 \mathrm{~Hz}, 1 \mathrm{H}, 4^{\prime}-$ H), 3.45 (s, $3 \mathrm{H}, \mathrm{OMe}), 3.46(\mathrm{dd}, J=3.7,9.0 \mathrm{~Hz}, 1 \mathrm{H}, \quad 3 '-\mathrm{H}), 3.47$ $\left(\mathrm{m}, 1 \mathrm{H}, 1-\mathrm{H}_{\mathrm{a}}\right), 3.47$ (s, $\left.3 \mathrm{H}, \mathrm{OMe}\right), 3.49$ (s, $\left.3 \mathrm{H}, \mathrm{OMe}\right), 3.51-3.58$ (m, $\left.4 \mathrm{H}, 66^{\prime}-\mathrm{H}_{2}, 5^{\prime}-\mathrm{H}, 2^{\prime}-\mathrm{H}\right), 3.76$ (ddd, $J=6.2,6.2,9.9 \mathrm{~Hz}, 1 \mathrm{H}$, $\left.1-\mathrm{H}_{\mathrm{b}}\right), 4.19$ (ddd, $\left.J=6.3,6.3,11.1 \mathrm{~Hz}, 1 \mathrm{H}, 3-\mathrm{H}_{\mathrm{a}}\right), 4.24(\mathrm{ddd}, J=$ $\left.6.4,6.4,11.0 \mathrm{~Hz}, 1 \mathrm{H}, 3-\mathrm{H}_{\mathrm{b}}\right), 4.85\left(\mathrm{~d}, J=1.8 \mathrm{~Hz}, 1 \mathrm{H}, 1^{\prime}-\mathrm{H}\right) \mathrm{ppm}$. ${ }^{1} \mathrm{H}$ NMR (500 MHz, $\mathrm{C}_{6} \mathrm{D}_{6}$ ): $\delta=1.64$ (qui, $J=6.5 \mathrm{~Hz}, 2 \mathrm{H}, 2-\mathrm{H}_{2}$ ), 1.68 (s, $3 \mathrm{H}, \mathrm{OMe}$ ), 3.21 (ddd, $J=6.2,6.2,9.8 \mathrm{~Hz}, 1 \mathrm{H}, 1-\mathrm{H}_{\mathrm{a}}$ ), 3.23 (s, $3 \mathrm{H}, \mathrm{OMe}), 3.23(\mathrm{~s}, 3 \mathrm{H}, \mathrm{OMe}), 3.26$ (s, $3 \mathrm{H}, \mathrm{OMe}), 3.46$ (s, 3 $\mathrm{H}, \mathrm{OMe}), 3.49\left(\mathrm{dd}, J=2.0,2.8 \mathrm{~Hz}, 1 \mathrm{H}, 2^{\prime}-\mathrm{H}\right), 3.62(\mathrm{dd}, J=2.0$, $\left.10.5 \mathrm{~Hz}, 1 \mathrm{H}, 6^{\prime}-\mathrm{H}_{\mathrm{a}}\right), 3.66\left(\mathrm{dd}, J=5.5,10.5 \mathrm{~Hz}, 1 \mathrm{H}, 6^{\prime}-\mathrm{H}_{\mathrm{b}}\right), 3.67$ $\left(\mathrm{dd}, J=3.0,9.0 \mathrm{~Hz}, 1 \mathrm{H}, 3^{\prime}-\mathrm{H}\right), 3.71$ (ddd, $J=6.5,6.5,9.5 \mathrm{~Hz}, 1$ $\left.\mathrm{H}, 1-\mathrm{H}_{\mathrm{b}}\right), 3.72\left(\mathrm{dd}, J=9.3,9.3 \mathrm{~Hz}, 1 \mathrm{H}, 4^{\prime}-\mathrm{H}\right), 3.83$ (ddd, $J=2.0$, $\left.5.5,9.3 \mathrm{~Hz}, 1 \mathrm{H}, 5^{\prime}-\mathrm{H}\right), 4.03(\mathrm{ddd}, J=6.4,6.4,11.0 \mathrm{~Hz}, 1 \mathrm{H}, 3-$ $\left.\mathrm{H}_{\mathrm{a}}\right), 4.07\left(\mathrm{ddd}, J=6.3,6.3,11.0 \mathrm{~Hz}, 1 \mathrm{H}, 2-\mathrm{H}_{\mathrm{b}}\right), 4.81(\mathrm{~d}, J=1.9$ $\left.\mathrm{Hz}, 1 \mathrm{H}, 11^{\prime}-\mathrm{H}\right) \mathrm{ppm} .{ }^{13} \mathrm{C}$ NMR $\left(125.7 \mathrm{MHz}, \mathrm{CDCl}_{3}\right): \delta=20.8$ $\left(\mathrm{CH}_{3}, \mathrm{OAc}\right), 28.6\left(\mathrm{CH}_{2}, \mathrm{C}-2\right), 57.7\left(\mathrm{CH}_{3}, \mathrm{OMe}\right), 58.9\left(\mathrm{CH}_{3}, \mathrm{OMe}\right)$,

$59.1\left(\mathrm{CH}_{3} \mathrm{OMe}\right), 60.5\left(\mathrm{CH},{ }_{3} \mathrm{OMe}\right), 61.4(\mathrm{CH}, \mathrm{C}-3), 63.9(\mathrm{CH}, 2$ C-1), $71.4\left(\mathrm{CH}, \mathrm{C}-5^{\prime}\right), 71.7\left(\mathrm{CH}_{2}, \mathrm{C}^{\prime} 6^{\prime}\right), 76.4\left(\mathrm{CH}, \mathrm{C}-4{ }^{\prime}\right), 77.1(\mathrm{CH}$, C-2'), $81.2\left(\mathrm{CH}, \mathrm{C}-3^{\prime}\right), 96.9\left(\mathrm{CH}, \mathrm{C}-1^{\prime}\right), 171.0(\mathrm{C}, \mathrm{OAc}) \mathrm{ppm} .{ }^{13} \mathrm{C}$ NMR (125.7 MHz, $\left.\mathrm{C}_{6} \mathrm{D}_{6}\right): \delta=20.4\left(\mathrm{CH}_{3}, \mathrm{OAc}\right), 29.0\left(\mathrm{CH}_{2}, \mathrm{C}-2\right)$, $57.2\left(\mathrm{CH}_{3}, \mathrm{OMe}\right), 58.9\left(\mathrm{CH}_{3}, \mathrm{OMe}\right), 59.0\left(\mathrm{CH}_{3}, \mathrm{OMe}\right), 60.5\left(\mathrm{CH}_{3}\right.$ OMe $), 61.3\left(\mathrm{CH}_{2}, \mathrm{C}-3\right), 64.0\left(\mathrm{CH}_{2}, \mathrm{C}-1\right), 72.6\left(\mathrm{CH}_{2}, \mathrm{C}-6{ }^{\prime}\right), 72.8$ (CH, C-5'), $77.2(\mathrm{CH}, \mathrm{C}-4$ '), 77.5 (CH, C-2'), 82.6 (CH, C-3'), 98.1 (CH, C-1'), 170.0 (C, OAc) ppm. IR (film): $\tilde{v}=1739 \mathrm{~cm}^{-1}$. MS $(70 \mathrm{eV}, \mathrm{EI}): m / z(\%)=235(<1)\left[M-\mathrm{C}_{5} \mathrm{H}_{9} \mathrm{O}_{2}\right]^{+}, 219(1), 161$ (11), 101 (100). HRMS (EI): $m / z$ : calcd. for $\mathrm{C}_{10} \mathrm{H}_{19} \mathrm{O}_{6}: 235.1182[M-$ $\left.\mathrm{C}_{5} \mathrm{H}_{9} \mathrm{O}_{2}\right]^{+}$; found 235.1152. $\mathrm{C}_{15} \mathrm{H}_{28} \mathrm{O}_{8}$ (336.38): calcd. C 53.56, H 8.39; found C 53.50, H 8.49.

Compound 38: $[\alpha]_{\mathrm{D}}=-58.3\left(c=0.060\right.$, in $\left.\mathrm{CHCl}_{3}\right) .{ }^{1} \mathrm{H}$ NMR $\quad(500$ $\left.\mathrm{MHz}, \mathrm{CDCl}_{3}\right): \delta=1.89-1.99\left(\mathrm{~m}, 2 \mathrm{H}, 2-\mathrm{H}_{2}\right), 2.04(\mathrm{~s}, 3 \mathrm{H}$, OAc), $3.17\left(\mathrm{dd}, J=3.2,9.0 \mathrm{~Hz}, 1 \mathrm{H}, 3^{\prime}-\mathrm{H}\right), 3.26$ (ddd, $J=1.8,5.6$, $\left.9.5 \mathrm{~Hz}, 1 \mathrm{H}, 5^{\prime}-\mathrm{H}\right), 3.32$ (dd, $\left.J=9.1,9.5 \mathrm{~Hz}, 1 \mathrm{H}, 4^{\prime}-\mathrm{H}\right), 3.39$ (s, 3 $\mathrm{H}, \mathrm{OMe}), 3.48$ (s, $3 \mathrm{H}, \mathrm{OMe}), 3.49\left(\mathrm{~m}, 1 \mathrm{H}, 1-\mathrm{H}_{\mathrm{a}}\right), 3.51(\mathrm{~s}, 3 \mathrm{H}$, OMe), $3.58\left(\mathrm{dd}, J=5.9,10.7 \mathrm{~Hz}, 1 \mathrm{H}, 6^{\prime}-\mathrm{H}_{\mathrm{a}}\right), 3.61$ (s, $\left.3 \mathrm{H}, \mathrm{OMe}\right)$, $3.66\left(\mathrm{dd}, J=1.9,10.2 \mathrm{~Hz}, 1 \mathrm{H}, 6^{\prime}-\mathrm{H}_{\mathrm{b}}\right), 3.66(\mathrm{~d}, J=3.5 \mathrm{~Hz}, 1 \mathrm{H}$, $\left.2^{\prime}-\mathrm{H}\right), 4.02$ (ddd, $\left.J=5.9,5.9,9.7 \mathrm{~Hz}, 1 \mathrm{H}, 1-\mathrm{H}_{\mathrm{b}}\right), 4.14$ (ddd, $J=$ $\left.6.2,6.3,11.0 \mathrm{~Hz}, 1 \mathrm{H}, 3-\mathrm{H}_{\mathrm{a}}\right), 4.19(\mathrm{ddd}, J=6.3,6.3,11.0 \mathrm{~Hz}, 1 \mathrm{H}$, $\left.3-\mathrm{H}_{\mathrm{b}}\right), 4.36\left(\mathrm{~s}, 1 \mathrm{H}, 11^{\prime}-\mathrm{H}\right) \mathrm{ppm} .{ }^{13} \mathrm{C} \mathrm{NMR}\left(125.7 \mathrm{MHz}, \mathrm{CDCl}_{3}\right): \delta$ $=20.9\left(\mathrm{CH}_{3}, \mathrm{OAc}\right), 28.9\left(\mathrm{CH}_{2}, \mathrm{C}-2\right), 57.4\left(\mathrm{CH}_{3}, \mathrm{OMe}\right), 59.3\left(\mathrm{CH}_{3}\right.$, $\mathrm{OMe}), 60.7\left(\mathrm{CH}_{3}, \mathrm{OMe}\right), 61.5\left(\mathrm{CH}_{2}, \mathrm{C}-3\right), 61.7\left(\mathrm{CH}_{3}, \mathrm{OMe}\right), 66.3$ $\left(\mathrm{CH}_{2}, \mathrm{C}-1\right), 72.0\left(\mathrm{CH}_{2}, \mathrm{C}^{\prime} 6^{\prime}\right), 75.6\left(\mathrm{CH}, \mathrm{C}-5^{\prime}\right), 76.5\left(\mathrm{CH}, \mathrm{C}-4^{\prime}\right)$, $76.879\left(\mathrm{CH}, \mathrm{C}-2^{\prime}\right), 83.9\left(\mathrm{CH}, \mathrm{C}-3^{\prime}\right), 101.6\left(\mathrm{CH}, \mathrm{C}-1^{\prime}\right), 171.1(\mathrm{C}$, OAc) ppm. IR (film): $\tilde{v}=1737 \mathrm{~cm}^{-1}$. MS $(70 \mathrm{eV}, \mathrm{EI}): \mathrm{m} / \mathrm{z}(\%)=$ $235(<1)\left[M-\mathrm{C}_{5} \mathrm{H}_{9} \mathrm{O}_{2}\right]^{+}, 219(<1), 187$ (1), $101(89), 88(100)$. 
HRMS (EI): $m / z$ : calcd. for $\mathrm{C}_{10} \mathrm{H}_{19} \mathrm{O}_{6}: 235.1182\left[M \mathrm{C}_{5} \mathrm{H}_{9} \mathrm{O}_{2}\right]^{+}$; found 235.1197. $\mathrm{C}_{15} \mathrm{H}_{28} \mathrm{O}_{8}$ (336.38): calcd. C 53.56, $\mathrm{H} \mathrm{8.39;} \mathrm{found}$ C 53.41, H 8.15.

Method B ( $\left.n \mathrm{Bu}_{3} \mathrm{SnD} / \mathrm{AIBN}\right)$ : A solution of phthalimide $35(120 \mathrm{mg}$, $0.273 \mathrm{mmol})$ in dry benzene $(20.5 \mathrm{~mL})$ containing $n \mathrm{Bu}_{3} \mathrm{SnD}(74 \mu \mathrm{L}$, $0.273 \mathrm{mmol}$ ) and AIBN (4.5 mg, $0.027 \mathrm{mmol})$ was heated at reflux temperature for $3.5 \mathrm{~h}$. After this time another portion of $n \mathrm{Bu} \mathrm{SnD}$ ( $74 \mu \mathrm{L}, 0.273 \mathrm{mmol})$ and AIBN (4.5 $\mathrm{mg}, 0.027 \mathrm{mmol})$ were added and heating at reflux was continued for an additional $1.25 \mathrm{~h}$. After cooling to room temperature the reaction mixture was concentrated under reduced pressure. The residue was dissolved in $\mathrm{CH}_{3} \mathrm{CN}$, washed with $n$-hexane and the combined more polar extracts were concentrated under reduced pressure. Acetylation of the crude residue in dry pyridine $(3 \mathrm{~mL})$ containing $\mathrm{Ac}_{2} \mathrm{O}(1 \mathrm{~mL})$ at room temperature for $4 \mathrm{~h}$ gave after silica gel column chromatography (hexanes/EtOAc, 65:35 < 55:45) the following compounds (in order of elution): 3 -acetyloxypropyl $2,3,4,6$-tetra- $O$-methyl- $\beta$ - $\square-\left(5-{ }^{2} \mathrm{H}\right)$ gulopyranoside (39) (11.7 $\mathrm{mg}, 0.035 \mathrm{mmol}, 13 \%)$, 3-acetyloxypropyl 2,3,4,6-tetra- $O$-methyl- $\alpha-\square-\left[5-{ }^{2} \mathrm{H}\right]$ mannopyranoside (40) $(27.5 \mathrm{mg}$, $0.082 \mathrm{mmol}, 30 \%,{ }^{1} \mathrm{H} /{ }^{2} \mathrm{H}$ ratio, 7:3), and 3-acetyloxypropyl 2,3,4,6tetra- $O$-methyl- $\beta-\square-\left(1-{ }^{2} \mathrm{H}\right)$ mannopyranoside $(\mathbf{4 1})(13 \mathrm{mg}, 0.038$ mmol, $14 \%)$, all as colourless oils.

Compound 39: ${ }^{1} \mathrm{H}$ NMR $\left(500 \mathrm{MHz}, \mathrm{CDCl}_{3}\right): \delta=1.89-1.95(\mathrm{~m}, 2$ $\left.\mathrm{H}, 2-\mathrm{H}_{2}\right), 2.03$ (s, $\left.3 \mathrm{H}, \mathrm{OMe}\right), 3.26$ (dd, $J=3.1,8.0 \mathrm{~Hz}, 1 \mathrm{H}, 2^{\prime}-$ $\mathrm{H}), 3.37$ (d, $\left.J=3.4 \mathrm{~Hz}, 1 \mathrm{H}, 4^{\prime}-\mathrm{H}\right), 3.39$ (s, $\left.3 \mathrm{H}, \mathrm{OMe}\right), 3.44$ (s, 3 $\mathrm{H}, \mathrm{OMe}), 3.47$ (s, $3 \mathrm{H}, \mathrm{OMe}), 3.50$ (d, $J=10.3 \mathrm{~Hz}, 1 \mathrm{H}, 6^{\prime}-\mathrm{H}_{\mathrm{a}}$ ), $3.52(\mathrm{~s}, 3 \mathrm{H}, \mathrm{OMe}), 3.58\left(\mathrm{~d}, J=9.5 \mathrm{~Hz}, 1 \mathrm{H}, 6^{\prime}-\mathrm{H}_{\mathrm{b}}\right), 3.58$ (ddd, $J$ $\left.=6.5,6.5,9.5 \mathrm{~Hz}, 1 \mathrm{H}, 1-\mathrm{H}_{\mathrm{a}}\right), 3.76\left(\mathrm{dd}, J=3.4,3.4 \mathrm{~Hz}, 1 \mathrm{H}, 3^{\prime}-\right.$ $\mathrm{H}), 3.95$ (ddd, $\left.J=6.1,6.1,9.5 \mathrm{~Hz}, 1 \mathrm{H}, 1-\mathrm{H}_{\mathrm{b}}\right), 4.15(\mathrm{t}, J=6.1 \mathrm{~Hz}$, $\left.2 \mathrm{H}, 3-\mathrm{H}_{2}\right), 4.65\left(\mathrm{~d}, J=8.0 \mathrm{~Hz}, 1 \mathrm{H}, 11^{\prime}-\mathrm{H}\right) \mathrm{ppm} .{ }^{13} \mathrm{C} \mathrm{NMR}$ $\left(125.7 \mathrm{MHz}, \mathrm{CDCl}_{3}\right): \delta=20.9\left(\mathrm{CH}_{3}, \mathrm{OAc}\right), 29.0\left(\mathrm{CH}_{2}, \mathrm{C}-2\right), 58.8$ $\left(\mathrm{CH}_{3}, \mathrm{OMe}\right), 59.1\left(\mathrm{CH}_{3}, \mathrm{OMe}\right), 59.2\left(\mathrm{CH}_{3}, \mathrm{OMe}\right), 59.6\left(\mathrm{CH}_{3}\right.$, $\mathrm{OMe}), 61.7\left(\mathrm{CH}_{2}, \mathrm{C}-3\right), 65.8\left(\mathrm{CH}_{2}, \mathrm{C}-1\right), 70.933\left(\mathrm{CH}_{2}, \mathrm{C}-6^{\prime}\right)$, $75.893\left(\mathrm{CH}, \mathrm{C}-4^{\prime}\right), 76.7\left(\mathrm{CH}, \mathrm{C}-3^{\prime}\right), 78.2\left(\mathrm{CH}, \mathrm{C}-2^{\prime}\right), 100.8(\mathrm{CH}$, C-1'), 171.1 (C, OAc) ppm. MS (70 eV, EI): $m / z(\%)=220(<1)$ $\left[M-\mathrm{C}_{5} \mathrm{H}_{9} \mathrm{O}_{3}\right]^{+}, 188(1), 101$ (77), 88 (100). HRMS (EI): $\mathrm{m} / z$ : calcd. for $\mathrm{C}_{10} \mathrm{H}_{18}{ }^{2} \mathrm{HO}_{5}$ : $220.1295\left[M-\mathrm{C}_{5} \mathrm{H}_{9} \mathrm{O}_{3}\right]^{+}$; found 220.1262 .

Compound 40: ${ }^{1} \mathrm{H}$ NMR $\left(500 \mathrm{MHz}, \mathrm{C} \mathrm{D}_{6}\right){ }_{6} \delta=1.64$ (qui, $J=$ $6.5 \mathrm{~Hz}, 2 \mathrm{H}, 2-\mathrm{H}), 1.68$ (s, $3 \mathrm{H}, \mathrm{OAc}$ ), 3.21 (ddd, $J=6.2,6.2$, $9.8 \mathrm{~Hz}, 1 \mathrm{H}, 1-\mathrm{H}_{\mathrm{a}}$ ), 3.24 (s, $3 \mathrm{H}, \mathrm{OMe}$ ), 3.25 (s, $\left.3 \mathrm{H}, \mathrm{OMe}\right), 3.27$ (s, $3 \mathrm{H}, \mathrm{OMe}), 3.48$ (s, $3 \mathrm{H}, \mathrm{OMe}), 3.49(\mathrm{dd}, J=2.0,2.8 \mathrm{~Hz}, 1 \mathrm{H}$, $\left.2^{\prime}-\mathrm{H}\right), 3.63\left(\mathrm{dd}, J=2.0,10.5 \mathrm{~Hz}, 1 \mathrm{H}, 6^{\prime}-\mathrm{H}_{\mathrm{a}}\right), 3.66(\mathrm{dd}, J=5.5$, $\left.10.5 \mathrm{~Hz}, 1 \mathrm{H}, 66^{\prime}-\mathrm{H}_{\mathrm{b}}\right), 3.69-3.75\left(\mathrm{~m}, 3 \mathrm{H}, 4^{\prime}-\mathrm{H}, 33^{\prime}-\mathrm{H}, 1-\mathrm{H}_{\mathrm{b}}\right), 3.84$ (ddd, $J=2.0,5.5,9.3 \mathrm{~Hz}, 1 \mathrm{H}, 5^{\prime}-\mathrm{H}$ ), 4.03 (ddd, $J=6.4,6.4$, $11.0 \mathrm{~Hz}, 1 \mathrm{H}, 3-\mathrm{H}_{\mathrm{a}}$ ), 4.07 (ddd, $J=6.3,6.3,11.0 \mathrm{~Hz}, 1 \mathrm{H}, 3-\mathrm{H}_{\mathrm{b}}$ ), $4.82\left(\mathrm{~d}, J=1.8 \mathrm{~Hz}, 1 \mathrm{H}, 1{ }^{\prime}-\mathrm{H}\right) \mathrm{ppm} .{ }^{13} \mathrm{C} \mathrm{NMR}\left(125.7 \mathrm{MHz}, \mathrm{C}_{6} \mathrm{D}_{6}\right)$ : $\delta=20.4\left(\mathrm{CH}_{3}, \mathrm{OAc}\right), 29.0\left(\mathrm{CH}_{2}, \mathrm{C}-2\right), 57.2\left(\mathrm{CH}_{3}, \mathrm{OMe}\right), 58.9$ $\left(\mathrm{CH}_{3}, \mathrm{OMe}\right), 59.0\left(\mathrm{CH}_{3}, \mathrm{OMe}\right), 60.5\left(\mathrm{CH}_{3}, \mathrm{OMe}\right), 61.3\left(\mathrm{CH}_{2}, \mathrm{C}-\right.$ 3), $64.0\left(\mathrm{CH}_{2}, \mathrm{C}-1\right), 72.547\left(\mathrm{CH}_{2}, \mathrm{C}-6\right.$ '-D $), 72.607\left(\mathrm{CH}_{2}, \mathrm{C}-6{ }^{\prime}-\mathrm{H}\right)$, 72.8 (CH, C-5'), 77.112 (CH, C-4'-D), 77.173 (CH, C-4'-H), 77.5 (CH, C-2'), $82.6\left(\mathrm{CH}, \mathrm{C}-3^{\prime}\right), 98.1\left(\mathrm{CH}, \mathrm{C}-1^{\prime}\right), 170.0(\mathrm{C}, \mathrm{OAc}) \mathrm{ppm}$. MS (70 eV, EI): $m / z(\%)=220(<1)\left[M-\mathrm{C}_{5} \mathrm{H}_{9} \mathrm{O}_{3}\right]^{+}, 219(<1), 188$ (1.2), 187 (1.3), 101 (100). HRMS (EI): $m / z$ : calcd. for $\mathrm{C}_{10} \mathrm{H}_{18}{ }^{2} \mathrm{HO}_{5}$ : $220.1295\left[M-\mathrm{C}_{5} \mathrm{H}_{9} \mathrm{O}_{3}\right]^{+}$; found 220.1274 .

Compound 41: ${ }^{1} \mathrm{H}$ NMR $(400 \mathrm{MHz}, \mathrm{CDCl})_{3} \delta=1.89-1.98(\mathrm{~m}, 2$ $\left.\mathrm{H}, 2-\mathrm{H}_{2}\right), 2.04$ (s, $\left.3 \mathrm{H}, \mathrm{OAc}\right), 3.17$ (dd, $\left.J=3.2,9.0 \mathrm{~Hz}, 1 \mathrm{H}, 3^{\prime}-\mathrm{H}\right)$, 3.26 (ddd, $\left.J=1.8,5.8,9.8 \mathrm{~Hz}, 1 \mathrm{H}, 5^{\prime}-\mathrm{H}\right), 3.32(\mathrm{dd}, J=8.7,9.5 \mathrm{~Hz}$, $\left.1 \mathrm{H}, 4^{\prime}-\mathrm{H}\right), 3.39$ (s, $\left.3 \mathrm{H}, \mathrm{OMe}\right), 3.48$ (s, $\left.3 \mathrm{H}, \mathrm{OMe}\right), 3.49$ (m, $1 \mathrm{H}$, $\left.1-\mathrm{H}_{\mathrm{a}}\right), 3.51$ (s, $\left.3 \mathrm{H}, \mathrm{OMe}\right), 3.58\left(\mathrm{dd}, J=6.1,10.3 \mathrm{~Hz}, 1 \mathrm{H}, 6^{\prime}-\mathrm{H}_{\mathrm{a}}\right.$ ), 3.61 (s, $3 \mathrm{H}, \mathrm{OMe}), 3.65\left(\mathrm{dd}, J=1.9,10.6 \mathrm{~Hz}, 1 \mathrm{H}, 6^{\prime}-\mathrm{H}_{\mathrm{b}}\right), 3.66$ $\left(\mathrm{d}, J=3.2 \mathrm{~Hz}, 1 \mathrm{H}, 2^{\prime}-\mathrm{H}\right), 4.02(\mathrm{ddd}, J=5.8,5.8,9.5 \mathrm{~Hz}, 1 \mathrm{H}, 1-$ $\left.\mathrm{H}_{\mathrm{b}}\right), 4.13\left(\mathrm{ddd}, J=6.4,6.4,11.1 \mathrm{~Hz}, 1 \mathrm{H}, 3-\mathrm{H}_{\mathrm{a}}\right), 4.18(\mathrm{ddd}, J=$
6.6, 6.6, $\left.11.1 \mathrm{~Hz}, 1 \mathrm{H}, 3-\mathrm{H}_{\mathrm{b}}\right) \mathrm{ppm} .{ }^{13} \mathrm{C} \mathrm{NMR}\left(100.6 \mathrm{MHz}, \mathrm{CDCl}_{3}\right)$ : $\delta=20.9\left(\mathrm{CH}_{3}, \mathrm{OAc}\right), 28.9\left(\mathrm{CH}_{2}, \mathrm{C}-2\right), 57.3\left(\mathrm{CH}_{3}, \mathrm{OMe}\right), 59.2$ $\left(\mathrm{CH}_{3}, \mathrm{OMe}\right), 60.7\left(\mathrm{CH}_{3}, \mathrm{OMe}\right), 61.5\left(\mathrm{CH}_{2}, \mathrm{C}-3\right), 61.7\left(\mathrm{CH}_{3}, \mathrm{OMe}\right)$, $66.2\left(\mathrm{CH}_{2}, \mathrm{C}-1\right), 71.9\left(\mathrm{CH}_{2}, \mathrm{C}-6 \mathrm{\prime}\right), 75.6\left(\mathrm{CH}, \mathrm{C}-5{ }^{\prime}\right), 76.4(\mathrm{CH}, \mathrm{C}-$ 4'), 76.733 (CH, C-2'), 83.9 (CH, C-3'), 171.1 (C, OAc) ppm. MS $(70 \mathrm{eV}, \mathrm{EI}): m / z(\%)=236(<1)\left[M-\mathrm{C}_{5} \mathrm{H}_{9} \mathrm{O}_{2}\right]^{+}, 220(1), 188$ (1), 162 (10), 101 (100). HRMS (EI): $m / z$ : calcd. for $\mathrm{C}_{10} \mathrm{H}_{18}{ }^{2} \mathrm{HO}_{6}$ : $236.1244\left[\mathrm{M}-\mathrm{C}_{5} \mathrm{H}_{9} \mathrm{O}_{2}\right]^{+}$; found 236.1247 .

Oxidative HAT of 2,6-Anhydro-7,8,9-trideoxy-1,3,4,5-tetra- $O$ methyl-D-glycero-D-manno-decitol (31): A solution of alcohol 31 (18 mg, $0.062 \mathrm{mmol})$ in $\mathrm{CH}_{2} \mathrm{Cl}_{2}(3.5 \mathrm{~mL})$ containing DIB (34 mg, $0.105 \mathrm{mmol}$ ) and iodine $(16 \mathrm{mg}, 0.062 \mathrm{mmol})$ under nitrogen was irradiated with two $80 \mathrm{~W}$ tungsten-filament lamps at room temperature for $1 \mathrm{~h}$. The reaction mixture was then poured into $10 \%$ aqueous $\mathrm{Na}_{2} \mathrm{~S}_{2} \mathrm{O}_{3}$ and extracted with $\mathrm{CH}_{2} \mathrm{Cl}_{2}$, dried with $\mathrm{Na}_{2} \mathrm{SO}_{4}$, and concentrated. Chromatotron chromatography of the reaction residue (hexanes/EtOAc, 85:15) gave in order of elution 1,5-anhydro-4,4-diiodo-2,3,4-trideoxy-6,7,8,10-tetra- $O$-methyl- $\alpha$ - $\square$-mannodec-5-ulopyranose (34a) (4.6 mg, $0.009 \mathrm{mmol}, 14 \%$ ), 1,5-anhydro2,3,4-trideoxy-6,7,8,10-tetra- $O$-methyl- $\alpha$ - $\square$-manno-dec-5-ulopyranose $(\mathbf{3 2 \alpha})(4.3 \mathrm{mg}, 0.015 \mathrm{mmol}, 24 \%), 1,5$-anhydro-2,3,4-trideoxy-4-iodo-6,7,8,10-tetra- $O$-methyl- $\square$-glycero- $\beta$ - $\square$-talo-dec-5-ulopyranose (33ß) (4.5 mg, $0.011 \mathrm{mmol}, 18 \%$ ), and 1,5-anhydro-2,3,4trideoxy- $6,7,8,10$-tetra- $O$-methyl- $\beta$ - $\square$-manno-dec-5-ulopyranose (32ß) $(5.6 \mathrm{mg}, 0.019 \mathrm{mmol}, 31 \%)$ as colourless oils.

Compound 32 $\alpha:[\alpha]_{\mathrm{D}}=+73.2\left(c=0.220\right.$, in $\left.\mathrm{CHCl}_{3}\right) .{ }^{1} \mathrm{H}$ NMR $(500$ $\left.\mathrm{MHz}, \mathrm{CDCl}_{3}\right): \delta=1.37(\mathrm{ddd}, J=4.2,13.2,13.2 \mathrm{~Hz}, 1 \mathrm{H}, 1-$

$\left.\mathrm{H}_{\mathrm{a}}\right), 1.48-1.62\left(\mathrm{~m}, 3 \mathrm{H}, 3-\mathrm{H}_{2}, 2-\mathrm{H}_{\mathrm{a}}\right), 1.81$ (ddddd, $J=3.9,5.9,13.4$, $\left.13.4,13.4 \mathrm{~Hz}, 1 \mathrm{H}, 2-\mathrm{H}_{\mathrm{b}}\right), 2.06\left(\mathrm{~m}, 1 \mathrm{H}, 1-\mathrm{H}_{\mathrm{b}}\right), 3.33(\mathrm{~d}, J=3.1 \mathrm{~Hz}$, $\left.1 \mathrm{H}, 2^{\prime}-\mathrm{H}\right), 3.36\left(\mathrm{dd}, J=9.5,9.5 \mathrm{~Hz}, 1 \mathrm{H}, 4^{\prime}-\mathrm{H}\right), 3.40$ (s, $3 \mathrm{H}$, OMe), 3.46 (ddd, $\left.J=2.0,5.3,10.1 \mathrm{~Hz}, 1 \mathrm{H}, 5^{\prime}-\mathrm{H}\right), 3.49$ (s, $3 \mathrm{H}$, OMe), 3.51 (s, 3 H, OMe), 3.54 (s, 3 H, OMe), 3.54-3.63 (m, $5 \mathrm{H}$, $\left.6^{\prime}-\mathrm{H}_{2}, 4-\mathrm{H}_{2}, 3^{\prime}-\mathrm{H}\right) \mathrm{ppm} .{ }^{13} \mathrm{C}$ NMR $\left(100.6 \mathrm{MHz}, \mathrm{CDCl}_{3}\right): \delta=18.4$ $\left(\mathrm{CH}_{2}, \mathrm{C}-2\right), 25.1\left(\mathrm{CH}_{2}, \mathrm{C}-3\right), 31.2\left(\mathrm{CH}_{2}, \mathrm{C}-1\right), 58.0\left(\mathrm{CH}_{3}, \mathrm{OMe}\right)$, $59.4\left(\mathrm{CH}_{3}, \mathrm{OMe}\right), 60.5\left(\mathrm{CH}_{3}, \mathrm{OMe}\right), 60.6\left(\mathrm{CH}_{2}, \mathrm{C}-4\right), 61.8\left(\mathrm{CH}_{3}\right.$, OMe), 71.6 (CH, C-5'), $72.1\left(\mathrm{CH}_{2}, \mathrm{C}^{-6} \mathbf{6}^{\prime}\right), 76.8$ (CH, C-4'), 81.2 $\left(\mathrm{CH}, \mathrm{C}-2^{\prime}\right), 82.7\left(\mathrm{CH}, \mathrm{C}-3^{\prime}\right), 98.2\left(\mathrm{C}, \mathrm{C}-1^{\prime}\right) \mathrm{ppm}$. IR (film): $\tilde{v}=$ $2825 \mathrm{~cm}^{-1}$. MS (70 eV, EI): $\mathrm{m} / z(\%)=290(<1)[\mathrm{M}]^{+}, 289(3), 257$ (1), 225 (1), 101 (59). HRMS (EI): $m / z$ : calcd. for $\mathrm{C}_{14} \mathrm{H}_{26} \mathrm{O}_{6}$ : $290.1729[\mathrm{M}]^{+}$; found 290.1720. C $\mathrm{H}_{2} \mathrm{O}_{6}(290.36)$ : calcd. C 57.91, H 9.03; found C 57.93, H 8.90.

Compound 32 $\beta:[\alpha]_{\mathrm{D}}=-62.6\left(c=0.700\right.$, in $\left.\mathrm{CHCl}_{3}\right) .{ }^{1} \mathrm{H} \mathrm{NMR} \quad(500$ $\mathrm{MHz}, \mathrm{CDCl}_{3}$ ): $\delta=1.38$ (ddd, $J=4.2,13.4,13.4 \mathrm{~Hz}, 1 \mathrm{H}, 1-$

$\left.\mathrm{H}_{\mathrm{a}}\right), 1.51-1.72\left(\mathrm{~m}, 4 \mathrm{H}, 3-\mathrm{H}_{2}, 2-\mathrm{H}_{2}\right), 1.95$ (ddd, $J=2.8,2.8$, $\left.13.7 \mathrm{~Hz}, 1 \mathrm{H}, 1-\mathrm{H}_{\mathrm{b}}\right), 3.35$ (dd, $\left.J=8.7,8.7 \mathrm{~Hz}, 1 \mathrm{H}, 4^{\prime}-\mathrm{H}\right), 3.35$ (d, $\left.J=3.4 \mathrm{~Hz}, 1 \mathrm{H}, 2^{\prime}-\mathrm{H}\right), 3.40$ (s, $\left.3 \mathrm{H}, \mathrm{OMe}\right), 3.41$ (m, $2 \mathrm{H}, 5^{\prime}-\mathrm{H}, 3^{\prime}-$ $\mathrm{H}), 3.47$ (s, $3 \mathrm{H}, \mathrm{OMe}), 3.49$ (s, $3 \mathrm{H}, \mathrm{OMe}), 3.58$ (s, $3 \mathrm{H}, \mathrm{OMe})$, $3.60\left(\mathrm{dd}, J=6.2,10.6 \mathrm{~Hz}, 1 \mathrm{H}, 6^{\prime}-\mathrm{H}_{\mathrm{a}}\right), 3.66(\mathrm{dd}, J=2.5,10.6 \mathrm{~Hz}$, $\left.1 \mathrm{H}, 6{ }^{\prime}-\mathrm{H}_{\mathrm{b}}\right), 3.70$ (ddd, $\left.J=2.5,2.5,11.2 \mathrm{~Hz}, 1 \mathrm{H}, 4-\mathrm{H}_{\mathrm{a}}\right), 4.04$ (ddd, $\left.J=3.1,12.0,12.0 \mathrm{~Hz}, 1 \mathrm{H}, 4-\mathrm{H}_{\mathrm{b}}\right) \mathrm{ppm} .{ }^{13} \mathrm{C} \mathrm{NMR}(125.7 \mathrm{MHz}$, $\left.\mathrm{CDCl}_{3}\right): \delta=17.7\left(\mathrm{CH}_{2}, \mathrm{C}-3\right), 25.1\left(\mathrm{CH}_{2}, \mathrm{C}-2\right), 28.2\left(\mathrm{CH}_{2}, \mathrm{C}-1\right)$, $57.7\left(\mathrm{CH}_{3}, \mathrm{OMe}\right), 59.2\left(\mathrm{CH}_{3}, \mathrm{OMe}\right), 60.3\left(\mathrm{CH}_{3}, \mathrm{OMe}\right), 61.9\left(\mathrm{CH}_{2}\right.$, C-4), $61.9\left(\mathrm{CH}_{3}, \mathrm{OMe}\right), 72.8\left(\mathrm{CH}_{2}, \mathrm{C}-6{ }^{\prime}\right), 73.6\left(\mathrm{CH}, \mathrm{C}-5^{\prime}\right), 77.0$ $(\mathrm{CH}), 80.5(\mathrm{CH}), 81.8\left(\mathrm{CH}, \mathrm{C}-3^{\prime}\right), 98.4\left(\mathrm{C}, \mathrm{C}-1^{\prime}\right) \mathrm{ppm}$. IR (film): v $=2830,1107 \mathrm{~cm}^{-1}$. MS $(70 \mathrm{eV}, \mathrm{EI}): \mathrm{m} / \mathrm{z}(\%)=245$ (8) $[\mathrm{M}$ - $\left.\mathrm{C}_{2} \mathrm{H}_{5} \mathrm{O}\right]^{+}, 213$ (4), 153 (19), 101 (100). HRMS (EI): $\mathrm{m} / z$ :calcd. for $\mathrm{C}_{12} \mathrm{H} \mathrm{Q}: 245.1389\left[M-\mathrm{C} \mathrm{H}_{2} \mathrm{Ol}_{5}^{+}\right.$; found 245.1391. C H $\mathrm{Q}_{26} 6$ (290.36): calcd. C 57.91, H 9.03; found C 57.93, H 8.98.

Compound 33ß: $[\alpha]_{\mathrm{D}}=-22.3\left(c=0.430\right.$, in $\left.\mathrm{CHCl}_{3}\right) .{ }^{1} \mathrm{H}$ NMR $\quad(500$ $\left.\mathrm{MHz}, \mathrm{CDCl}_{3}\right): \delta=1.35-1.39\left(\mathrm{~m}, 1 \mathrm{H}, 3-\mathrm{H}_{\mathrm{a}}\right), 1.83$ (ddddd, $J$ $\left.=4.4,4.4,13.2,13.2,13.2 \mathrm{~Hz}, 1 \mathrm{H}, 3-\mathrm{H}_{\mathrm{b}}\right), 2.25-2.30(\mathrm{~m}, 1 \mathrm{H}, 2-$ $\left.\mathrm{H}_{\mathrm{a}}\right), 2.55$ (dddd, $\left.J=3.9,12.9,12.9,12.9 \mathrm{~Hz}, 1 \mathrm{H}, 2-\mathrm{H}_{\mathrm{b}}\right), 3.39(\mathrm{~s}, 3$ 
$\mathrm{H}, \mathrm{OMe}$ ), 3.46 (s, $3 \mathrm{H}, \mathrm{OMe}), 3.49$ (s, $3 \mathrm{H}, \mathrm{OMe}), 3.51$ (s, $3 \mathrm{H}$, OMe), 3.67-3.72 (m, $\left.3 \mathrm{H}, 6^{\prime}-\mathrm{H}_{\mathrm{a}}, 4-\mathrm{H}_{\mathrm{a}}, 4^{\prime}-\mathrm{H}\right), 3.74$ (dd, $J=4.8$, $\left.4.8 \mathrm{~Hz}, 1 \mathrm{H}, 3^{\prime}-\mathrm{H}\right), 3.77$ (dd, $\left.J=5.9,10.1 \mathrm{~Hz}, 1 \mathrm{H}, 6^{\prime}-\mathrm{H}_{\mathrm{b}}\right), 3.95(\mathrm{~d}$, $\left.J=5.0 \mathrm{~Hz}, 1 \mathrm{H}, 2^{\prime}-\mathrm{H}\right), 3.99\left(\mathrm{ddd}, J=5.6,5.6,5.6 \mathrm{~Hz}, 1 \mathrm{H}, 5^{\prime}-\mathrm{H}\right)$, 4.07 (ddd, $J=2.5,11.2,13.2 \mathrm{~Hz}, 1 \mathrm{H}, 4-\mathrm{H}_{\mathrm{b}}$ ), 4.63 (dd, $J=4.5$, $12.9 \mathrm{~Hz}, 1 \mathrm{H}, 1-\mathrm{H}) \mathrm{ppm} .{ }^{13} \mathrm{C} \mathrm{NMR}\left(125.7 \mathrm{MHz}, \mathrm{CDCl}_{3}\right): \delta=28.7$ $\left(\mathrm{CH}_{2}, \mathrm{C}-3\right), 32.3(\mathrm{CH}, \mathrm{C}-1), 33.2\left(\mathrm{CH}_{2}, \mathrm{C}-2\right), 57.7\left(\mathrm{CH}_{3}, \mathrm{OMe}\right)$, $57.8\left(\mathrm{CH}_{3}, \mathrm{OMe}\right), 58.8\left(\mathrm{CH}_{3}, \mathrm{OMe}\right), 59.3\left(\mathrm{CH}_{3}, \mathrm{OMe}\right), 60.5\left(\mathrm{CH}_{2}\right.$, C-4), $\left.72.1\left(\mathrm{CH}_{2}, \mathrm{C}^{-6}\right)^{\prime}\right), 72.9\left(\mathrm{CH}, \mathrm{C}-5^{\prime}\right), 76.3(\mathrm{CH}, \mathrm{C}-3 '), 76.7(\mathrm{CH}$, C-4'), 77.4 (CH, C-2'), 97.2 (C, C-1') ppm. IR (film): $\tilde{v}=2929$, 2827, 1454, 1193, $1101 \mathrm{~cm}^{-1}$. MS $(70 \mathrm{eV}, \mathrm{EI}): \mathrm{m} / \mathrm{z}(\%)=416(2)$ $[\mathrm{M}]^{+}, 371$ (1), 289 (8), 257 (4). HRMS (EI): $\mathrm{m} / \mathrm{z}$ : calcd. for $\mathrm{C}_{14} \mathrm{H}_{25} \mathrm{IO}_{6}$ : $416.0696[\mathrm{M}]^{+}$; found 416.0688. $\mathrm{C}_{14} \mathrm{H}_{25} \mathrm{IO}_{6}$ (416.25): calcd. C 40.40, H 6.05; found C 40.65, H 5.96.

Compound 34 $\alpha:[\alpha]_{\mathrm{D}}=+36.4\left(c=0.220\right.$, in $\left.\mathrm{CHCl}_{3}\right) .{ }^{1} \mathrm{H}$ NMR $\quad(500$ $\left.\mathrm{MHz}, \mathrm{CDCl}_{3}\right): \delta=1.12\left(\mathrm{~m}, 1 \mathrm{H}, 3-\mathrm{H}_{\mathrm{a}}\right), 2.14$ (ddddd, $J=3.5$, $6.2,12.3,12.3,14.1 \mathrm{~Hz}, 1 \mathrm{H}, 3-\mathrm{H}_{\mathrm{b}}$ ), 2.67 (dddd, $J=1.5,3.2,3.2$, $14.4 \mathrm{~Hz}, 1 \mathrm{H}, 2-\mathrm{H}_{\mathrm{a}}$ ), 3.08 (ddd, $J=3.8,12.6,14.7 \mathrm{~Hz}, 1 \mathrm{H}, 2-\mathrm{H}_{\mathrm{b}}$ ), 3.42-3.44 (m, 2 H, 5'-H, 4'-H), 3.49 (s, 3 H, OMe), 3.51 (s, 3 H, $\mathrm{OMe}$ ), 3.53 (s, $3 \mathrm{H}, \mathrm{OMe}), 3.56\left(\mathrm{dd}, J=2.9,9.4 \mathrm{~Hz}, 1 \mathrm{H}, 3^{\prime}-\mathrm{H}\right)$, 3.60-3.70 (m, $\left.4 \mathrm{H}, 6^{\prime}-\mathrm{H}_{2}, 4-\mathrm{H}_{2}\right), 3.78$ (s, $\left.3 \mathrm{H}, \mathrm{OMe}\right), 4.05$ (d, $J=$ $\left.2.9 \mathrm{~Hz}, 1 \mathrm{H}, 2^{\prime}-\mathrm{H}\right) \mathrm{ppm} .{ }^{13} \mathrm{C} \mathrm{NMR}\left(125.7 \mathrm{MHz}, \mathrm{CDCl}_{3}\right): \delta=7.8$ (C, C-1) $27.0\left(\mathrm{CH}_{2}, \mathrm{C}-3\right), 50.1\left(\mathrm{CH}_{2}, \mathrm{C}-2\right), 58.1\left(\mathrm{CH}_{3}, \mathrm{OMe}\right), 60.1$ $\left(\mathrm{CH}_{2}, \mathrm{C}-4\right), 60.1\left(\mathrm{CH}_{3}, \mathrm{OMe}\right), 60.7\left(\mathrm{CH}_{3}, \mathrm{OMe}\right), 61.0\left(\mathrm{CH}_{3}, \mathrm{OMe}\right)$, $72.1\left(\mathrm{CH}_{2}, \mathrm{C}^{-} 6^{\prime}\right), 74.0\left(\mathrm{CH}, \mathrm{C}-5^{\prime}\right), 75.7\left(\mathrm{CH}, \mathrm{C}-4{ }^{\prime}\right), 81.6(\mathrm{CH}, \mathrm{C}-$ $\left.2^{\prime}\right), 84.2\left(\mathrm{CH}, \mathrm{C}-3^{\prime}\right), 96.7$ (C, C-1') ppm. IR (film): $\tilde{v}=2925,2852$, $1456,1196,1103 \mathrm{~cm}^{-1}$. MS $(70 \mathrm{eV}, \mathrm{EI}): \mathrm{m} / \mathrm{z}(\%)=542(6)[\mathrm{M}]^{+}, 415$ (53), 288 (100), 253 (23). HRMS (EI): $m / z$ : calcd. for $\mathrm{C}_{14} \mathrm{H}_{24} \mathrm{I}_{2} \mathrm{O}_{6}$ :

$541.9662[\mathrm{M}]^{+}$; found 541.9666. C H I O $(542.45)$ : calcd. C 31.02, H 4.46; found C 31.25, H 4.46.

Reductive HAT of 2,6-Anhydro-7,8,9-trideoxy-1,3,4,5-tetra- $O$ methyl-10-O-N-phthalimido-D-glycero-D-manno-decitol (42). Method A $\left(n \mathrm{Bu}_{3} \mathrm{SnH} / \mathrm{AIBN}\right): \mathrm{A}$ solution of phthalimide $\mathbf{4 2}(29 \mathrm{mg}$, $0.066 \mathrm{mmol})$ in dry benzene $(5 \mathrm{~mL})$ containing $n \mathrm{Bu}_{3} \mathrm{SnH} \quad(18 \mu \mathrm{L}$, $0.066 \mathrm{mmol}$ ) and AIBN (1 $\mathrm{mg}, 0.007 \mathrm{mmol}$ ) was heated at reflux temperature for $1 \mathrm{~h}$. After cooling to room temperature the reaction mixture was concentrated under reduced pressure. The residue was dissolved in $\mathrm{CH}_{3} \mathrm{CN}$, washed with $n$-hexane and the combined more polar extracts were concentrated under reduced pressure. The residue was purified by column chromatography (hexanes/EtOAc, $25: 75)$ to give in order of elution the $\beta$-alcohol $\mathbf{4 3}$ (7.8 mg, $0.027 \mathrm{mmol}, 40 \%)$ and the $\alpha$-alcohol $31(11.4 \mathrm{mg}, 0.039 \mathrm{mmol}, 59$ $\%)$ which had physical and spectroscopic properties identical to those of the product prepared in the Supporting Information.

Compound 43: $[\alpha]_{\mathrm{D}}=-22.5\left(c=0.40\right.$, in $\left.\mathrm{CHCl}_{3}\right) .{ }^{1} \mathrm{H}$ NMR $(500$ $\left.\mathrm{MHz}, \mathrm{CDCl}_{3}\right): \delta=1.40-1.62\left(\mathrm{~m}, 5 \mathrm{H}, 3-\mathrm{H}_{2}, 2-\mathrm{H}_{2}, 1-\mathrm{H}_{\mathrm{a}}\right), 1.82$ (m, $\left.1 \mathrm{H}, 1-\mathrm{H}_{\mathrm{b}}\right), 3.18$ (dd, $\left.J=3.1,9.5 \mathrm{~Hz}, 1 \mathrm{H}, 3^{\prime}-\mathrm{H}\right), 3.20-3.24$ (m, $\left.2 \mathrm{H}, 5^{\prime}-\mathrm{H}, 11^{\prime}-\mathrm{H}\right), 3.34$ (dd, $\left.J=9.5,9.5 \mathrm{~Hz}, 1 \mathrm{H}, 4^{\prime}-\mathrm{H}\right), 3.37$ (s, 3 $\mathrm{H}, \mathrm{OMe}), 3.47\left(\mathrm{dd}, J=0.6,2.8 \mathrm{~Hz}, 1 \mathrm{H}, 2^{\prime}-\mathrm{H}\right), 3.511(\mathrm{~s}, 3 \mathrm{H}$, OMe), 3.513 (s, $3 \mathrm{H}, \mathrm{OMe}$ ), 3.52 (dd, $J=6.4,10.4 \mathrm{~Hz}, 1 \mathrm{H}, 6^{\prime}-$ $\left.\mathrm{H}_{\mathrm{a}}\right), 3.57(\mathrm{~s}, 3 \mathrm{H}, \mathrm{OMe}), 3.62\left(\mathrm{dd}, J=2.0,10.6 \mathrm{~Hz}, 1 \mathrm{H}, 6^{\prime}-\mathrm{H}_{\mathrm{b}}\right)$, 3.64 (ddd, $J=2.2,6.4,6.4 \mathrm{~Hz}, 2 \mathrm{H}, 4-\mathrm{H}_{2}$ ) ppm. ${ }^{13} \mathrm{C} \mathrm{NMR}$ $\left(100.6 \mathrm{MHz}, \mathrm{CDCl}_{3}\right): \delta=22.1\left(\mathrm{CH}_{2}, \mathrm{C}-2\right), 30.6\left(\mathrm{CH}_{2}, \mathrm{C}-1\right), 32.5$ $\left(\mathrm{CH}_{2}, \mathrm{C}-3\right), 57.7\left(\mathrm{CH}_{3}, \mathrm{OMe}\right), 59.1\left(\mathrm{CH}_{3}, \mathrm{OMe}\right), 60.6\left(\mathrm{CH}_{3}, \mathrm{OMe}\right)$, $61.6\left(\mathrm{CH}_{3}, \mathrm{OMe}\right), 62.5\left(\mathrm{CH}_{2}, \mathrm{C}-4\right), 72.2\left(\mathrm{CH}_{2}, \mathrm{C}-6{ }^{\prime}\right), 77.0(\mathrm{CH}, \mathrm{C}-$ 4'), $77.523\left(\mathrm{CH}, \mathrm{C}-2^{\prime}\right), 78.4\left(\mathrm{CH}, \mathrm{C}-2^{\prime}\right), 79.2\left(\mathrm{CH}, \mathrm{C}-5^{\prime}\right), 86.8(\mathrm{CH}$ C-3') ppm. IR (film): $\tilde{v}=3472 \mathrm{~cm}^{-1}$. MS $(70 \mathrm{eV}, \mathrm{EI}): \mathrm{m} / \mathrm{z}(\%)=$ $293(2)[\mathrm{M}+\mathrm{H}]^{+}, 275$ (23), $211(8), 101(100)$. HRMS (EI): $\mathrm{m} / z$ : calcd. for $\mathrm{C}_{1} \mathrm{O}_{29}: 293.1964[\mathrm{M}+\mathrm{H}]^{+}$; found 293.1957 . $\mathrm{C}_{14} \mathrm{H}_{28} \mathrm{O}_{6}$ (292.37): calcd. C 57.51, H 9.65; found C 57.54, H 9.57.

Method B ( $\left.n \mathrm{Bu}_{3} \mathrm{SnD} / \mathrm{AIBN}\right)$ : A solution of phthalimide $42(30 \mathrm{mg}$, $0.069 \mathrm{mmol})$ in dry benzene $(5.1 \mathrm{~mL})$ containing $n \mathrm{Bu} 3 \mathrm{SnD}(19 \mu \mathrm{L}$,
$0.069 \mathrm{mmol})$ and AIBN $(1.1 \mathrm{mg}, 0.007 \mathrm{mmol})$ was heated at reflux temperature for $1 \mathrm{~h}$. After cooling to room temperature the reaction mixture was concentrated under reduced pressure. The residue was dissolved in $\mathrm{CH}_{3} \mathrm{CN}$, washed with $n$-hexane and the combined more polar extracts were concentrated under reduced pressure. The residue was purified by silica gel column chromatography (hexanes/ EtOAc, 25:75) to give in order of elution 5,9-anhydro-2,3,4-trideoxy-6,7,8,10-tetra- $O$-methyl- $\square-\left(5-{ }^{2} \mathrm{H}\right)$ glycero- $\square$-galacto-decitol (44) $(6.5 \mathrm{mg}, 0.022 \mathrm{mmol}, 32 \%)$ and 2,6-anhydro-7,8,9-trideoxy-1,3,4,5-tetra- $O$-methyl- $\square-\left[7-{ }^{2} \mathrm{H}\right]$ glycero- $\square-m a n n o-d e c i t o l \quad(45)$ (12.3 mg, $0.042 \mathrm{mmol}, 61 \%,{ }^{1} \mathrm{H} /{ }^{2} \mathrm{H}$ ratio, $1: 4$ ).

Compound 44: ${ }^{1} \mathrm{H}$ NMR $(500 \mathrm{MHz}, \mathrm{CDCl})_{3} \delta=1.40-1.62(\mathrm{~m}, 5$ $\left.\mathrm{H}, 3-\mathrm{H}_{2}, 2-\mathrm{H}_{2}, 1-\mathrm{H}_{\mathrm{a}}\right), 1.82\left(\mathrm{~m}, 1 \mathrm{H}, 1-\mathrm{H}_{\mathrm{b}}\right), 3.18(\mathrm{dd}, J=3.1,9.5 \mathrm{~Hz}$, $\left.1 \mathrm{H}, 3^{\prime}-\mathrm{H}\right), 3.23$ (ddd, $\left.J=1.7,6.0,9.7 \mathrm{~Hz}, 1 \mathrm{H}, 5^{\prime}-\mathrm{H}\right), 3.34$ (dd, $J$ $\left.=9.5,9.5 \mathrm{~Hz}, 1 \mathrm{H}, 4^{\prime}-\mathrm{H}\right), 3.37(\mathrm{~s}, 3 \mathrm{H}, \mathrm{OMe}), 3.47(\mathrm{~d}, J=3.1 \mathrm{~Hz}$, $1 \mathrm{H}, 2^{\prime}-\mathrm{H}$ ), 3.511 (s, $3 \mathrm{H}, \mathrm{OMe}$ ), 3.513 (s, $3 \mathrm{H}, \mathrm{OMe}$ ), 3.52 (dd, $J$ $\left.=6.2,10.4 \mathrm{~Hz}, 1 \mathrm{H}, 6^{\prime}-\mathrm{H}_{\mathrm{a}}\right), 3.57(\mathrm{~s}, 3 \mathrm{H}, \mathrm{OMe}), 3.62(\mathrm{dd}, J=2.0$, $\left.10.6 \mathrm{~Hz}, 1 \mathrm{H}, 6{ }^{\prime}-\mathrm{H}_{\mathrm{b}}\right), 3.64\left(\mathrm{ddd}, J=2.2,6.4,6.4 \mathrm{~Hz}, 2 \mathrm{H}, 4-\mathrm{H}_{2}\right)$ ppm. ${ }^{13} \mathrm{C}$ NMR (125.7 MHz, $\left.\mathrm{CDCl}_{3}\right): \delta=22.1\left(\mathrm{CH}_{2}, \mathrm{C}-2\right), 30.486$ $\left(\mathrm{CH}_{2}, \mathrm{C}-1\right), 32.5\left(\mathrm{CH}_{2}, \mathrm{C}-3\right), 57.7\left(\mathrm{CH}_{3}, \mathrm{OMe}\right), 59.1\left(\mathrm{CH}_{3}, \mathrm{OMe}\right)$, $60.6\left(\mathrm{CH}_{3}, \mathrm{OMe}\right), 61.6\left(\mathrm{CH}_{3}, \mathrm{OMe}\right), 62.5\left(\mathrm{CH}_{2}, \mathrm{C}-4\right), 72.3\left(\mathrm{CH}_{2}\right.$, C-6'), 77.1 (CH, C-4'), $77.502\left(\mathrm{CH}, \mathrm{C}-2^{\prime}\right), 79.2\left(\mathrm{CH}, \mathrm{C}-5^{\prime}\right), 86.8$ $\left(\mathrm{CH}, \mathrm{C}-3^{\prime}\right) \mathrm{ppm}$. MS (70 eV, EI): $\mathrm{m} / z(\%)=276(10)[M-\mathrm{OH}]^{+}$, 257 (2), 101 (100). HRMS (EI): $m / z$ : calcd. for $\mathrm{C}_{14} \mathrm{H}_{26}{ }^{2} \mathrm{HO}_{5}$ : $276.1921[\mathrm{M}-\mathrm{OH}]^{+}$; found 276.1924 .

Compound 45: ${ }^{1} \mathrm{H}$ NMR $\left(500 \mathrm{MHz}, \mathrm{CDCl}_{3}\right): \delta=1.36-1.69(\mathrm{~m}, 5$ $\left.\mathrm{H}, 3-\mathrm{H}_{2}, 2-\mathrm{H}_{3} 1-\mathrm{H}\right), 3.34$ (dd, $\left.J=3.4,3.4 \mathrm{~Hz}, 1 \mathrm{H}, 2^{\prime}-\mathrm{H}\right), 3.36$ (s, $3 \mathrm{H}, \mathrm{OMe}), 3.37$ (m, $\left.1 \mathrm{H}, 4^{\prime}-\mathrm{H}\right), 3.40$ (s, $\left.3 \mathrm{H}, \mathrm{OMe}\right), 3.467$ (s, $3 \mathrm{H}$, OMe), 3.469 (s, $3 \mathrm{H}, \mathrm{OMe}), 3.48\left(\mathrm{~m}, 1 \mathrm{H}, 3^{\prime}-\mathrm{H}\right), 3.55-3.63$ (m, 5 $\left.\mathrm{H}, 6^{\prime}-\mathrm{H}_{2}, 5^{\prime}-\mathrm{H}, 4-\mathrm{H}_{2}\right), 3.94$ (dd, $\left.J=4.0,9.8 \mathrm{~Hz}, 1 \mathrm{H}, 1^{\prime}-\mathrm{H}\right) \mathrm{ppm}$. ${ }^{13} \mathrm{C}$ NMR (125.7 MHz, $\left.\mathrm{CDCl}_{3}\right): \delta=21.857\left(\mathrm{CH}_{2}, \mathrm{C}-2-\mathrm{D}\right), 21.953$ $\left(\mathrm{CH}_{2}, \mathrm{C}-2-\mathrm{H}\right), 28.681\left(\mathrm{CHD}, \mathrm{t},{ }^{1} J_{\mathrm{C}, \mathrm{D}}=19 \mathrm{~Hz}, \mathrm{C}-1-\mathrm{D}\right), 29.034$ $\left(\mathrm{CH}_{2}, \mathrm{C}-1-\mathrm{H}\right), 32.3\left(\mathrm{CH}_{2}, \mathrm{C}-3\right), 57.6\left(\mathrm{CH}_{3}, \mathrm{OMe}\right), 57.8\left(\mathrm{CH}_{3}\right.$, $\mathrm{OMe}), 59.1\left(\mathrm{CH}_{3}, \mathrm{OMe}\right), 59.7\left(\mathrm{CH}_{3}, \mathrm{OMe}\right), 62.6\left(\mathrm{CH}_{2}, \mathrm{C}-4\right), 71.8$ $\left(\mathrm{CH}_{2}, \mathrm{C}-6\right.$ '), $72.183\left(\mathrm{CH}, \mathrm{C}-1^{\prime}-\mathrm{D}\right), 72.247\left(\mathrm{CH}, \mathrm{C}-1^{\prime}-\mathrm{H}\right), 72.5(\mathrm{CH}$,

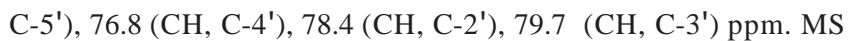
$(70 \mathrm{eV}, \mathrm{EI}): \mathrm{m} / z(\%)=293(1)[\mathrm{M}]^{+}, 269$ (7), 267 (5), 216 (7). HRMS (EI): $m / z$ : calcd. for $\mathrm{C}_{14} \mathrm{H}_{27}{ }^{2} \mathrm{HO}_{6}$ : 293.1949 [M]+; found 293.1953 .

Oxidative HAT of 5,9-Anhydro-2,3,4-trideoxy-6,7,8,10-tetra- $O$ methyl-D-glycero-D-galacto-decitol (43): A solution of alcohol 43 (25 mg, $0.086 \mathrm{mmol})$ in $\mathrm{CH}_{2} \mathrm{Cl}_{2}(3.5 \mathrm{~mL})$ containing DIB (47 mg, $0.146 \mathrm{mmol})$ and iodine $(22 \mathrm{mg}, 0.086 \mathrm{mmol})$ under nitrogen was irradiated with two $80 \mathrm{~W}$ tungsten-filament lamps at room temperature for $0.5 \mathrm{~h}$. The reaction mixture was then poured into $10 \%$ aqueous $\mathrm{Na}_{2} \mathrm{~S}_{2} \mathrm{O}_{3}$ and extracted with $\mathrm{CH}_{2} \mathrm{Cl}_{2}$, dried with $\mathrm{Na}_{2} \mathrm{SO}_{4}$, and concentrated. Chromatotron chromatography of the residue (hexanes/EtOAc, 7:3) gave in order of elution 1,5-anhydro-2,3,4trideoxy-6,7,8,10-tetra- $O$-methyl- $\alpha$ - $\square$-mano-dec-5-ulopyranose $\mathbf{3 2} \boldsymbol{\alpha}$ (7 mg, $0.024 \mathrm{mmol}, 28 \%$ ) and 1,5-anhydro-2,3,4-trideoxy-6,7,8,10tetra- $O$-methyl- $\beta-\square$-mano-dec-5-ulopyranose $\quad 32 \beta \quad(12.5 \mathrm{mg}$, $0.043 \mathrm{mmol}, 50 \%$ ), which had physical and spectroscopic properties identical to those of the products previously obtained (vide supra).

Reductive HAT of 5,9-Anhydro-2,3,4-trideoxy-6,7,8,10-tetra- $O$ methyl-1-O-phthalimido-D-glycero-D-galacto-decitol (46). Method A $\left(n \mathrm{Bu}_{3} \mathrm{SnH} / \mathrm{AIBN}\right)$ : A solution of phthalimide $46(47 \mathrm{mg}$,

$0.107 \mathrm{mmol})$ in dry benzene $(8.1 \mathrm{~mL})$ containing $n \mathrm{Bu} \mathrm{SnH}(29 \mu \mathrm{L}$, $0.107 \mathrm{mmol})$ and AIBN $(1.8 \mathrm{mg}, 0.011 \mathrm{mmol})$ was heated at reflux temperature for $1 \mathrm{~h}$. After cooling to room temperature the reaction mixture was concentrated under reduced pressure. The residue was dissolved in $\mathrm{CH}_{3} \mathrm{CN}$, washed with $n$-hexane and the combined more polar extracts were concentrated under reduced pressure. The 
residue was purified by column chromatography (hexanes/EtOAc, 3:7) to give the $\beta$-alcohol $\mathbf{4 3}$ (30 mg, $0.103 \mathrm{mmol}, 96 \%$ ) which had physical and spectroscopic properties identical to those of the product obtained in the Supporting Information.

Method B $\left(n \mathrm{Bu}_{3} \mathrm{SnD} / \mathrm{AIBN}\right)$ : A solution of phthalimide $46(60 \mathrm{mg}$, $0.137 \mathrm{mmol})$ in dry benzene (10.3) containing $n \mathrm{Bu}_{3} \mathrm{SnD}(37 \mu \mathrm{L}$, $0.137 \mathrm{mmol})$ and AIBN $(2.2 \mathrm{mg}, 0.014 \mathrm{mmol})$ was heated at reflux temperature under nitrogen for $1 \mathrm{~h}$. After this time another portion of $n \mathrm{Bu}_{3} \mathrm{SnH} \quad(18.5 \mu \mathrm{L}, \quad 0.068 \mathrm{mmol})$ and AIBN $(2.2 \mathrm{mg}$, $0.014 \mathrm{mmol}$ ) were added and heating at reflux was continued for an additional $0.5 \mathrm{~h}$. After cooling at room temperature the mixture was concentrated under reduced pressure. The residue was dissolved in $\mathrm{CH}$ CN, washed with $n$-hexane and the combined more polar extracts were concentrated under reduced pressure. The residue was purified by column chromatography (hexanes/EtOAc, 3:7), to give 5,9-anhydro-2,3,4-trideoxy-6,7,8,10-tetra- $O$-methyl- $\square$-(5${ }^{2} \mathrm{H}$ )-glycero- $\square$-galacto-decitol 44 (40 mg, $0.136 \mathrm{mmol}, 100 \%$ ) which had physical and spectroscopic properties identical to those of the product previously obtained (vide supra).

Supporting Information (see also the footnote on the first page of this article): A complete description of experimental details of precursors and analytical data for all new compounds can be found in the online version.

\section{Acknowledgments}

This work was supported by the Spanish Ministerio de Educación y Ciencia (MEC); Research Programs CTQ2004-06381/BQU, CTQ2004-02367/BQU and CTQ2007-67492/BQU, cofinanced by the Fondo Europeo de Desarrollo Regional (FEDER). I. P.-M. and L. M. Q. thank the CSIC (Program I3P) for fellowships.

1 a) P. Compain, V. Chagnault, O. R. Martin, Tetrahedron: Asymmetry 2009, 20, 677-711; b) K. W. Wellington, S. A. Benner, Nucleosides Nucleotides 2006, 25, 1309-1333; c) T. Bililign, B. R. Griffith, J. S. Thorson, Nat. Prod. Rep. 2005, 22, 742-760; d) D. Y. W. Lee, M. S. He, Curr. Top. Med. Chem. 2005, 5, 1333-1350; e) A. Dondoni, A. Marra, Chem. Rev. 2000, 100, 4395-4421; f) P. Vogel, R. Ferritto, K. Kraehenbuehl, A. Baudat, in: Carbohydrate Mimics: Concepts and Methods, Chapter 2, (Ed.: Y. Chapleur), Verlag Chemie, Weinheim, 1998, pp. 19-48; g) H. Togo, W. He, Y. Waki, M. Yokoyama, Synlett 1998, 700-717; h) Y. G. Du, R. J. Linhardt, I. R. Vlahov, Tetrahedron 1998, 54, 9913-9959; i) J.-M. Beau,

T. Gallagher, Top. Curr. Chem. 1997, 187, 1-54; j) F. Nicotra, Top. Curr. Chem. 1997, 187, 55-83; k) D. E. Levy, C. Tang, The Chemistry of C-Glycosides, Elsevier, Cambridge, 1995; 1) M. H. D. Postema, Tetrahedron 1992, 48, 8545-8599.

[2] For a recent review, see: a) J. Jiménez-Barbero, J. F. Espinosa, J. L. Asensio, F. J. Cañada, A. Poveda, Adv. Carbohydr. Chem. Biochem. 2000, 56, 235-284; b) A. Poveda, J. L. Asensio, T. Polat, H. Bazin, R. J. Linhardt, J. Jiménez-Barbero, Eur. J. Org. Chem. 2000, 1805-1813; c) J. L. Asensio, F. J. Cañada, X. H. Chen, N. Khan, D. R. Mootoo, J. Jiménez-Barbero, Chem. Eur. J. 2000, 6, 1035-1041; d) G. Rubinstenn, P. Sinaÿ, P. Berthault, J. Phys. Chem. A 1997, 101, 2536-2540; e) J. F. Espinosa, F. J. Cañada, J. L. Asensio, M. Martín-Pastor, H. Dietrich, M. Martín-Lomas, R. R. Schmidt, J. Jiménez-Barbero, J. Am. Chem. Soc. 1996, 118, 10862-10871; f) J. F. Espinosa, H. J. Dietrich, M. Martín-Lomas, R. R. Schmidt, J. Jiménez-Barbero, Tetrahedron Lett. 1996, 37, 1467-1470; g) J. F. Espinosa, F. J. Cañada, J. L. Asensio, H. Dietrich, M. Martín-Lomas, R. R. Schmidt, J. Jiménez-Barbero, Angew. Chem. Int. Ed. Engl. 1996, 35, 303-306; h) J. F. Espinosa, M. Martín-Pastor, J. L. Asensio, H. Dietrich, M. Martín-Lomas, R. R. Schmidt, J. Jim-
énez-Barbero, Tetrahedron Lett. 1995, 36, 6329-6332; i) A. Wei, A. Haudrechy, C. Audin, H.-S. Jun, N. Haudrechy-Bretel, Y. Kishi, J. Org. Chem. 1995, 60, 2160-2169 and references cited therein ; j) A. Wei, K. M. Boy, Y. Kishi, J. Am. Chem. Soc. 1995, 117, 9432-9437; k) Y. Wang, P. G. Goekjian, D. V. Ryckman, W. H. Miller, S. A. Babirad, Y. Kishi, J. Org. Chem. 1992, 57, 482-489; 1) S. A. Babirad, Y. Wang, P. G. Goekjian, Y. Kishi, J. Org. Chem. 1987, 52, 4825-4827.

3 C. G. Francisco, A. J. Herrera, A. R. Kennedy, D. Melián, E. Suárez, Angew. Chem. Int. Ed. 2002, 41, 856-858.

4 For other references on HAT reaction in disaccharide systems, see: a) A. Martín, I. Pérez-Martín, L. M. Quintanal, E. Suárez, Org. Lett. 2007, 9, 1785-1788; b) C. G. Francisco, A. J. Herrera, A. R. Kennedy, A. Martín, D. Melián, I. Pérez-Martín, L. M. Quintanal, E. Suárez, Chem. Eur. J. 2008, 14, 10369-

10381; c) A. Martín, I. Pérez-Martín, L. M. Quintanal, E. Suárez, J. Org. Chem. 2008, 73, 7710-7720.

5 MacroModel, version 9.7 and the AMBER* force field with the GB/SA solvent model for $\mathrm{CHCl}_{3}$, Schrödinger, LLC, New York, NY, 2009. The minimum for each conformational isomer was calculated performing a coordinate scan calculation of $\Phi$ and $\Psi$ dihedrals from -180 to $180^{\circ}$ in increments of $5^{\circ}$. See the corresponding contour plots at the Supporting Information section.

6 a) L. Feray, N. Kuznetsov, P. Renaud, in: Radicals in Organic Synthesis, vol. 2 (Eds.: P. Renaud, M. P. Sibi), Wiley-VCH, Weinheim, 2001, pp. 246-278; b) A. E. Dorigo, K. N. Houk, J. Am. Chem. Soc. 1987, 109, 2195-2197; c) A. E. Dorigo, K. N. Houk, J. Org. Chem. 1988, 53, 1650-1664; d) A. E. Dorigo, M. A. McCarrick, R. J. Loncharich, K. N. Houk, J. Am. Chem. Soc. 1990, 112, 7508-7514.

7 a) M. Upreti, D. Ruhela, R. A. Vishwakarma, Tetrahedron 2000, 56, 6577-6584; b) J. Kerékgyártó, J. P. Kamerling, J. B. Bouwstra, J. F. G. Vliegenthart, A. Liptak, Carbohydr. Res. 1989, 186, 51-62.

8 R. R. Schmidt, K.-H. Jung, in: Preparative Carbohydrate Chemistry (Ed.: S. Hanessian), Marcel Dekker, New York, 1997, pp. 283-312.

9 a) L. M. Mikkelsen, T. Skrydstrup, J. Org. Chem. 2003, 68, 2123-2128; b) B. Kuberan, S. A. Sikkander, H. Tomiyama, R. J. Linhardt, Angew. Chem. Int. Ed. 2003, 42, 2073-2075; c) S.Palmier, B. Vauzeilles, J.-M. Beau, Org. Biomol. Chem. 2003, 1, 1097-1098; d) L. M. Mikkelsen, S. L. Krintel, J. JiménezBarbero, T. Skrydstrup, J. Org. Chem. 2002, 67, 6297-6308; e) M. Ricci, L. Madariaga, T. Skrydstrup, Angew. Chem. Int. Ed. 2000, 39, 242-246; f) M. Ricci, P. Blakskjaer, T. Skrydstrup, $J$. Am. Chem. Soc. 2000, 122, 12413-12421; g) T. Skrydstrup, O. Jarreton, D. Mazéas, D. Urban, J.-M. Beau, Chem. Eur. J. 1998, 4, 655-671; h) O. Jarreton, T. Skrydstrup, J.-F. Espinosa, J. Jiménez-Barbero, J.-M. Beau, Chem. Eur. J. 1999, 5, 430441 ; i) O. Jarreton, T. Skrydstrup, J.-M. Beau, Chem. Commun. 1996, 1661-1662; j) D. Mazéas, T. Skrydstrup, J.-M. Beau, Angew. Chem. Int. Ed. Engl. 1995, 34, 909-912; k) P. de Pouilly, A. Chenede, J.-M. Mallet, P. Sinaÿ, Bull. Soc. Chim. Fr. 1993, 130, 256-265.

[10] a) R. R. Schmidt, R. Preuss, Tetrahedron Lett. 1989, 30, 3409

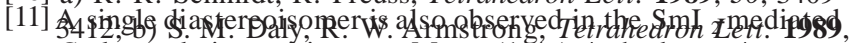
60 glyepsysation to give $\alpha-\square-M a n p-(1<4 a)-4 a-h y d r o x y-4 a-$ carba- $\alpha-\square$-Man $p$; see ref. ${ }^{[9 a]}$

12 a) O. Mitsunobu, Synthesis 1981, 1-28; b) E. Grochowski, J. Jurczak, Synthesis 1976, 682-684.

13 The deuterium position was determined by the coupling with the geminal carbon atom and also by the small yet significant displacement of the adjacent carbon signals in the ${ }^{13} \mathrm{C}$ NMR spectra, see: S. Berger, in: Encyclopedia of Nuclear Magnetic Resonance, vol. 2 (Eds.: D. M. Grant, R. K. Harris), John Wiley \& Sons, Chichester, 1996, pp. 1168-1172. 
14 For the synthesis of an spiroketal analogue, see: R. Preuss, K.H. Jung, R. R. Schmidt, Liebigs Ann. Chem. 1992, 377-382.

15 The methoxy groups at C-2' and C-3 were easily distinguished by $2 \mathrm{D}$ HSQC and HMBC correlations.

16 As expected, the preferred conformation of the rings is determined principally by the maximum number of anomeric effects a)P. Deslongchamps, Stereoelectronic Effects in Organic Chemistry, Pergamon Press, New York, 1983; b) P. Deslongchamps, D. D. Rowan, N. Pothier, T. Sauvé, J. K. Saunders, Can. J. Chem. 1981, 59, 1105-1121; c) N. Pothier, D. D. Rowan, P. Deslongchamps, J. K. Saunders, Can. J. Chem. 1981, 59, 11321139 .

17 Using a modified Karplus-type calculation as implemented in Maestro, version 9.0, Schrödinger, LLC, New York, NY, 2009.

18 Details on the preparation of these models are provided in the Supporting Information section.

19 A part of the present work has been published as a preliminary communication: A. Martín, L. M. Quintanal, E. Suárez, Tetrahedron Lett. 2007, 48, 5507-5511.

20 For convenience, the atom-numbering system used throughout this section and in the NMR assignments is the one depicted in the corresponding Scheme although a IUPAC systematic nomenclature has been used in the Experimental and Supporting Information sections.

21 a) W. N. Haworth, E. L. Hirst, J. A. B. Smith, J. Chem. Soc. 1930, 2659-2663; b) D. Crich, J.-T. Hwang, H. Yuan, J. Org. Chem. 1996, 61, 6189-6198.

22 Assignments were made by DEPT, COSY, HMBC and HSQC experiments. The anomeric stereochemistry was assigned on the basis of the ${ }^{33} J(\mathrm{H} 1, \mathrm{H} 2)$ and ${ }^{13} J(\mathrm{C} 1, \mathrm{H} 1)$ coupling constants and intramolecular NOE experiments. J. O. Duus, C. H. Gotfredsen, K. Bock, Chem. Rev. 2000, 100, 4589-4614 and references cited therein.

23 The high propensity of mannopyranosyl radicals for quenching by stannanes along the axial direction and formation of equatorial glycosides is well established. See, for example: D. Crich, S. Sun, J. Brunckova, J. Org. Chem. 1996, 61, 605-615 and references cited therein.

24 a) D. P. Curran, N. A. Porter, B. Giese, Stereochemistry of Radical Reactions, VCH, Weinheim, 1996, pp. 131-135; b) B. Giese, J. Dupuis, Angew. Chem. Int. Ed. Engl. 1983, 22, 622-623.

25 For studies on stereoelectronic effects in intermolecular hydrogen abstraction reactions see: a) V.Malatesta, K. U. Ingold, $J$.
Am. Chem. Soc. 1981, 103, 609-614; b) A. L. J. Beckwith, C. J. Easton, J. Am. Chem. Soc. 1981, 103, 615-619.

26 Radical-mediated intermolecular brominations can discriminate between $\mathrm{H}-5^{\prime}$ and $\mathrm{H}-1^{\prime}$, but the regioselectivity of the reaction is largely dependent on the anomeric substituent and configuration, as well as the halogenating agent used. In consequence, the regioselectivity results observed in these intermolecular reactions are not easily extrapolated to our models. In fact, a closely related compound, methyl 2,3,4,6-tetra- $O$-acetyl$\alpha-\square$-mannopyranoside, does not give any bromination and most of it was recovered unchanged after prolonged reaction times a) L. Somsák, R. J. Ferrier, Adv. Carbohyd. Chem. Biochem. 2000, 56, 65-151; b) J.-P. Praly, L. Brard, G. Descotes, L. Toupet, Tetrahedron 1989, 45, 4141-4152.

27 1,5-HAT is by far the most common intramolecular reaction when promoted by an alkoxy radical. However, examples of 1,6-hydrogen transfer (through a seven-membered transition state) from reactive carbons have also been described, high yields only being obtained in those cases when the hydrogen to be abstracted is bonded to an oxygen-substituted carbon: a) L. Feray, N. Kuznetsov, P. Renaud, in: Radicals in Organic Synthesis, Vol. 2 (Eds.: P. Renaud, M. P. Sibi), Wiley-VCH, Weinheim, 2001, pp. 246-278; b) J. Robertson, J. Pillai, R. K. Lush, Chem. Soc. Rev. 2001, 30, 94-103; c) R. L. Dorta, A. Martín, J. A. Salazar, E. Suárez, T. Prangé, J. Org. Chem. 1998, 63, 22512261; d) R. L. Dorta, A. Martín, J. A. Salazar, E. Suárez, T. Prangé, Tetrahedron Lett. 1996, 37, 6021-6024; e) R. L. Dorta, A. Martín, E. Suárez, T. Prangé, Tetrahedron: Asymmetry

1996, 7, 1907-1910; f) A. Martín, J. A. Salazar, E. Suárez, J. Org. Chem. 1996, 61, 3999-4006; g) A. Martín, J. A. Salazar, E. Suárez, Tetrahedron Lett. 1995, 36, 4489-4492; h) G. Majetich, K. Wheless, Tetrahedron 1995, 51, 7095-7129; i) S. J. Danishefsky, D. M. Armistead, F. E. Wincott, H. G. Selnick, R. Hungate, J. Am. Chem. Soc. 1987, 109, 8117-8119; j) I. T. Kay, D. Bartholomew, Tetrahedron Lett. 1984, 25, 2035-2038; k) J. I. Concepción, C. G. Francisco, R. Hernández, J. A. Salazar, E. Suárez, Tetrahedron Lett. 1984, 25, 1953-1956; 1) P. Brun, B. Waegell, in: Reactive Intermediates, vol. 3 (Ed.: R. A. Abramovitch), Plenum, New York, 1983, pp. 367-426.

Received: April 7, 2010

Published Online: August 5, 2010 